NOTE TO USERS

This reproduction is the best copy available.

(B)

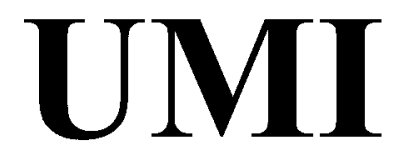


RYERSON UNIVERSITY

FACULTY OF GRADUATE STUDIES

IN ENGINEERING AND APPLIED SCIENCE

Department of Mechanical, Aerospace and Industrial Engineering

\title{
IMPLEMENTATION AND TESTING \\ OF A 3-D HYBRID RAPID PROTOTYPING SYSTEM CAPABLE OF \\ DEPOSITION AND MACHINING
}

\author{
Parham Ghasemi Madahian \\ ME8001 Mechanical Engineering - Project Report \\ Submitted in partial fulfillment \\ Of the requirements for the degree of \\ Master of Engineering
}

Faculty Supervisor: Dr. Vincent Chan

Date: August 4, 2003 
UMI Number: EC52972

\section{INFORMATION TO USERS}

The quality of this reproduction is dependent upon the quality of the copy submitted. Broken or indistinct print, colored or poor quality illustrations and photographs, print bleed-through, substandard margins, and improper alignment can adversely affect reproduction.

In the unlikely event that the author did not send a complete manuscript and there are missing pages, these will be noted. Also, if unauthorized copyright material had to be removed, a note will indicate the deletion.

(®)

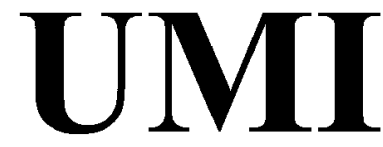

UMI Microform EC52972

Copyright 2008 by ProQuest LLC.

All rights reserved. This microform edition is protected against unauthorized copying under Title 17, United States Code.

ProQuest LLC

789 E. Eisenhower Parkway PO Box 1346

Ann Arbor, MI 48106-1346 


\section{Acknowledgments}

This project would not have been possible without the guidance, advice and understanding of Dr. Vincent Chan, the author's supervising Professor. I would like to sincerely thank Dr. Vincent Chan for his leadership, help and encouragement throughout this study. I would also like to thank Devin Ostrom (Technical officer at Ryerson University) for his expertise and technical support and Hassan Hamzeh for his previous work on the rapid prototyping system. This paper is dedicated to my parents, Reza and Gity, who have always supported me throughout my endeavours. 


\section{$\underline{\text { Abstract }}$}

Rapid Prototyping (RP) technology addresses the needs of industry to shorten design cycles and improve the design quality. In this project, an existing wax deposition system was retrofitted for wax extrusion of layers followed by surface and perimeter machining to ensure accuracy and quality. Work was undertaken to assess the influence of various parameters on the quality characteristics of the prototypes and to determine whether the modified machine would function to expectations. To carry out the experiments, a methodology and several computer programs were developed to translate a part's dimension into G-code notation. Shapes such as cubes, rectangular prisms, cylinders and pyramidal prototypes were produced from wax using the Hybrid Rapid Prototyping (HRP) system. Data relating to tolerance and accuracy was collected for the cubic/rectangular shapes as well as the circular/cylindrical shapes. The machine's accuracy (tolerance per inch) with respect to cubical shapes was measured at 0.018 " while the accuracy associated with cylindrical shapes was slightly less at 0.022 ". Furthermore, experimentation dealing with the benefits of combining machining and deposition rather than just deposition revealed that accuracies increased approximately 13 times with the addition of the milling head attachment. The evolution of prototype production from preliminary experiments was recorded, and modifications were made until the quality and dimensional accuracy were deemed to be satisfactory. The modifications made to the RP system significantly improved the accuracy and surface quality of the prototype parts. It is concluded that other standard RP systems can also be retrofitted to accommodate a machining station in order to improve accuracy and overall quality. 
Table of Contents

Page

Acknowledgments .........................................................................ii

Abstract ... ..................................................................................................

Table of Contents ........................................................................................

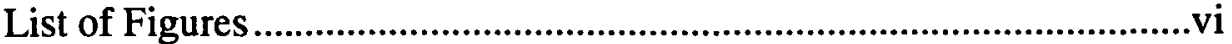

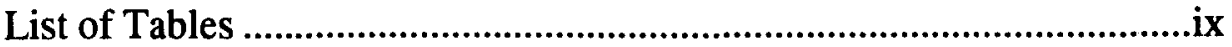

\section{CHAPTER 1 - INTRODUCTION}

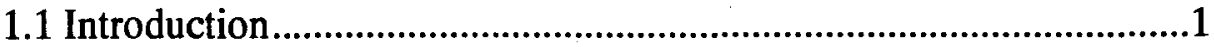

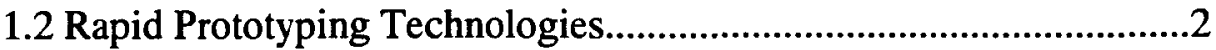

1.3 Other Hybrid Methods ................................................................. 7

1.4 Proposed Hybrid Design ............................................................11

\section{CHAPTER 2 - EXPERIMENTAL APPARATUS AND PROCEDURE}

2.1 Previous Rapid Prototyping Apparatus............................................14

2.2 Hybrid Rapid Prototyping Design I .........................................16

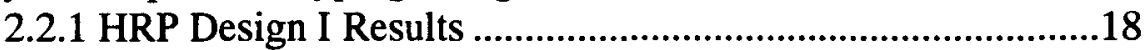

2.3 Hybrid Rapid Prototyping Design II...........................................19

2.3.1 HRP Design II Results .....................................................20

2.4 Hybrid Rapid Prototyping Design III ..............................................21

2.5 Testing Temperature, Gap size, Feed-rate and Pump-rate .................23

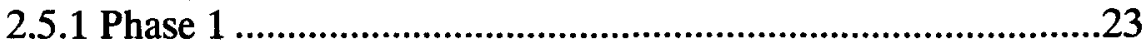

2.5.2 Phase 1 Results ............................................................25

2.5.3 Phase 1 Conclusion ...........................................................26

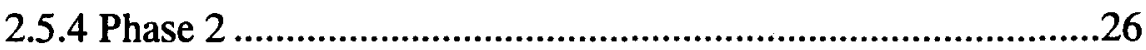

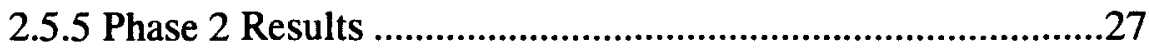

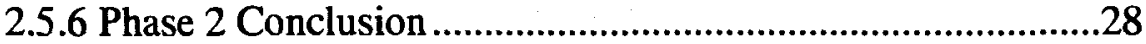

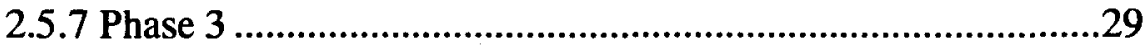

2.6 Fine Tuning Test Procedure ...........................................................31

2.6.1 Temperature Adjustment ....................................................31

2.6.2 Prototype Production Problems and Solutions ......................32

2.7 Designs and Implementation of Software .......................................34

2.8 Visual Illustration of Production Steps ..............................................38

\section{CHAPTER 3 - RESULTS AND DISCUSSION}

3.1 Cubic and Rectangular Prism Shapes ...........................................39

3.2 Circular and Cylindrical Shapes ..................................................43

3.3 Hollow Cubes and Pyramidal Shapes ............................................46 
CHAPTER 4 - CONCLUSIONS AND RECOMMENDATIONS

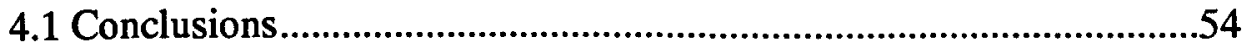

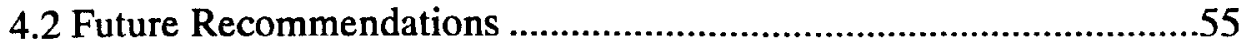

REFERENCES …...................................................................57

APPENDIX 1: AutoCAD Drawings for Original RP

Drawn by Hassan Hamzeh ...................................................................59

APPENDIX 2: Auto CAD Drawings for Hybrid RP ...........................80

APPENDIX 3: COMPUTER PROGRAMS ......................................84 


\section{List Of Figures}

$\begin{array}{lll}\text { Figure Description } & \text { Page }\end{array}$

$1 \quad$ Fused Deposition Modelling Diagram 2

2 Selective Laser Sintering Representation 3

3 Ballistic Particle Manufacturing Schematic 4

$4 \quad$ Three-Dimensional Printing Schematic 4

$5 \quad$ Laminated Object Manufacturing Diagram 5

6 ECLIPSE-RP Diagram 7

$\begin{array}{lll}7 & \text { Reverse Module Illustration } & 7\end{array}$

$8 \quad$ ECLIPSE-RP Photograph 9

$9 \quad$ Mould Fabrication Using SLS 9

$\begin{array}{lll}10 & \text { Robot Machining } & 10\end{array}$

$11 \quad$ I/O Board of HRP 12

$12 \quad$ Front View of RP Machine 14

13 Top View of RP Machine 14

14 Molten Wax Reservoir with Screw Pump 15

15 Blocks of Paraffin Wax Used 15

$16 \quad$ Z-axis DC Motor $\quad 16$

$17 \quad$ Z-axis Back Supports $\quad 16$

$18 \quad$ Custom Brass Collet 16

19 Custom Stainless Steel Protective Cover 17

$20 \quad$ Original Pump Motor 18

$21 \quad$ Original Speed Variable Control Box $\quad 18$ 


\section{List Of Figures Cont'd}

Figure Description $\quad$ Page

$22 \quad$ High Torque Replacement Motor 18

23 Second Speed Variable Control Box 19

$24 \quad$ New Wax Extrusion System 19

$25 \quad$ Digital Temperature Display 20

$26 \quad$ Hot Air Gun 22

27 Steel Pot Used For Melting Wax 22

28 Max Score/Temp. Vs. Temp. (1/8" gap, 1/8" Dia.) 24

29 Max Score/Temp. Vs. Temp. (1/16" gap, 1/16" Dia.) 28 (Metal Surface)

30 Max Score/Temp. Vs. Temp. (1/16" gap, 1/16" Dia.) 30 (Rough Paper Surface)

$31 \quad$ G-Code User Interface 34

32 Tool Path For A Solid Rectangular Shape 36

33 Step 1: Applying Adhesive 38

$34 \quad$ Step 2: Clamping Surface $\quad 38$

35 Step 3: Deposition Phase 38

$36 \quad$ Step 4: Curing The Wax 38

37 Step 5: End-mill position $\quad 38$

$38 \quad$ Step 6: Machining Perimeter $\quad 38$

$39 \quad$ Step 7: Machining Surface Phase 38

$40 \quad$ Step 8: Preparing Layer Bondage 38

$41 \quad$ View of Square Prototypes 41

vii 


\section{List Of Figures Cont'd}

Figure Description $\quad$ Page

$42 \quad$ Angled View of Square Prototypes 41

43 View of Rectangular Prism 42

$44 \quad$ One-layered Circular Prototypes 45

45 Multi-layered Circular Prototypes 45

46 Top View of Pyramidal Shape 46

47 Side View of Pyramidal Shape 46

48 Top View of Hollow Square 46

49 Zoom-in View of Pyramid Shape 47

$50 \quad$ Zoom-in View of Hollow Pyramid Shape 47

$51 \quad$ Genisys Commercial System 50

$52 \quad$ FDM 1650 Commercial System $\quad 50$

viii 


\section{List Of Tables}

Table

Description

Page

1

RP Technology Comparison

6

2

Score Sheet Based on 1/8" Nozzle Diameter

And Metallic Table Surface

Score Sheet Based on 1/16" Nozzle Diameter

And Metallic Table Surface

And Rough Paper Surface

Stepper Motor Speeds and Feeds

Prototype Results For Cubical Shapes

8

Prototype Results For Cylindrical Shapes

Between HRP and RP

9

Comparison Between The HRP And

Commercial RP Systems Under $\$ 60,000$ US

Comparison Between The HRP And
Commercial RP Systems Over $\$ 100,000$ US 


\section{CHAPTER 1 - INTRODUCTION}

\subsection{Introduction}

Rapid Prototyping (RP), also referred to as Layered Manufacturing, is a widely used method of manufacturing in product design and development, which has been increasingly applied in the process of design and development of new products. For automatic creation of physical prototypes, three fundamentally different methods are possible, namely incremental building, decremental building and deforming [1]. Among these, incremental building is commercially available in layered manufacturing systems and the other methods (decremental building and deforming) are available in conventional production systems like $\mathrm{CNC}$ milling machines and press machines. In recent years, the layered manufacturing process has found more widespread use due to its ability to produce internal cavities. As well, RP can produce a prototype very early in the product development cycle, without specialized tooling, and enhance the design process by providing rapid and effective feedback to the designer for visualization and verification. RP can dramatically shrink the time and expense required to bring a new product from initial concept to production. Over the past few years, a variety of new rapid manufacturing technologies have emerged; Stereolithography, Selective Laser Sintering (SLS), Fused Deposition Manufacturing (FDM), Laminated Object Manufacturing (LOM), Ballistic Particle Manufacturing (BPM), and Three Dimensional Printing (3D Printing). These technologies are capable of directly generating physical objects from CAD databases [1]. They all have a common important feature: adding materials rather than removing materials to produce the prototype part. This simplifies the $3 \mathrm{D}$ part producing process to $2 \mathrm{D}$ layer adding processes. This permits a part to be produced directly from its computer model. 


\subsection{Rapid Prototyping Technologies}

A number of RP technologies exist today. A discussion of the merits of each of these technologies follows.

\section{Stereolithography Apparatus (SLA)}

The SLA machine creates a prototype by tracing a layer of cross-sections on the surface of the liquid photopolymer pool with a laser beam. The laser beam is deflected horizontally along the $\mathrm{X}$ and $\mathrm{Y}$-axes by galvanometer driven mirrors so that it moves across the surface of the resin to produce a solid pattern. After a layer is built, the elevator drops a specified distance and a new coating of liquid resin covers the solidified layer. When all layers are completed, the prototype is about $95 \%$ cured. Post-curing is needed to completely solidify the prototype. This is done in a fluorescent oven where ultraviolet light floods the object. The accuracy achieved is about $0.1 \%$ of the overall dimension and deteriorates with larger sizes but no more than $0.5 \%$. The layer thickness is between 0.004 and $0.03 "$ [2].

\section{Fused Deposition Modelling (FDM)}

In an FDM process (Fig 1), a spool of thermoplastic filament feeds into a heated FDM

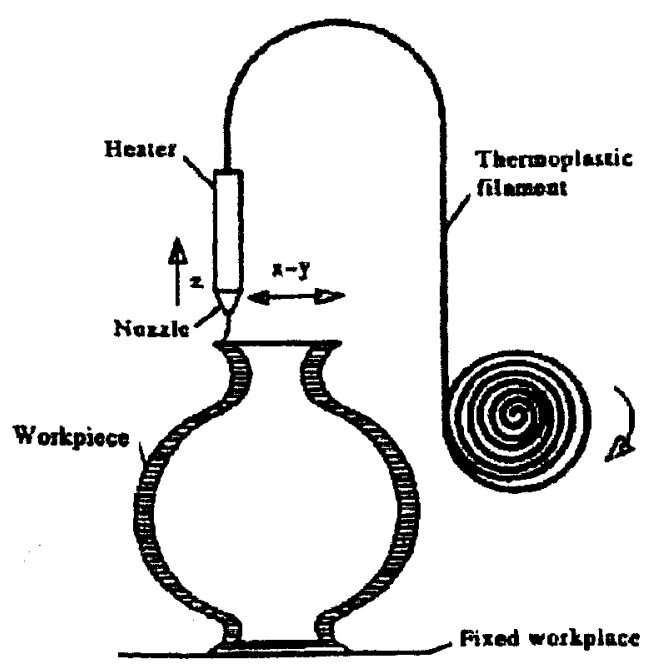

Fig 1: FDM Diagram [2] extrusion head. A computer controls the movement of the FDM head. Inside the flying extrusion head, the filament is melted into liquid by a resistant heater. The head traces an exact outline of each cross-section layer of the part. As the head moves horizontally along the $\mathrm{X}$ and $\mathrm{Y}$ axes the thermoplastic material is 
extruded out a nozzle by a precision pump. After one layer is finished, the extrusion head moves up a programmed distance in the $\mathrm{z}$ direction for building the next layer. Each layer is bonded to the previous layer through thermal heating. The overall tolerance is plus or minus $0.005 "$. Successive laminations are within the range of $0.001-0.05 "$, and wall thickness ranges from 0.01 to $0.25 "$ [2].

\section{Selective Laser Sintering (SLS)}

An SLS system uses a carbon dioxide laser to sinter successive layers of powder instead of liquid. In SLS processes, a thin layer of powder is applied by a counterrotating roller mechanism onto the work place. The powder material is preheated to a temperature slightly below its melting point. The laser beam traces the crosssection on the powder surface to heat up

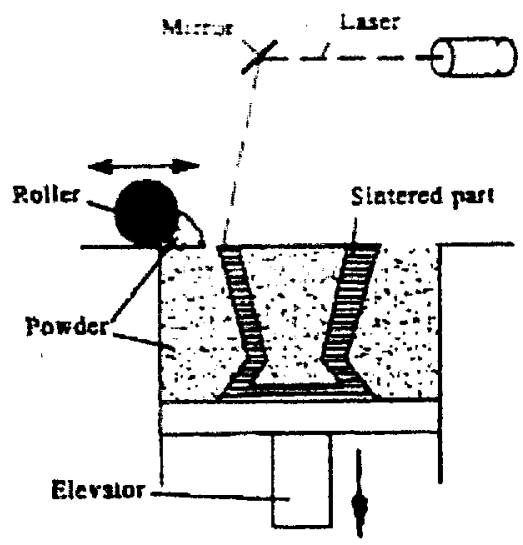
the powder to the sintering temperature so

Fig 2: SLS Representation [2] that the powder scanned by the laser is bonded (see Fig 2). When a layer of the crosssection is completed, the roller levels another layer of powder over the sintered one for the next pass. The average accuracy achieved ranges from 0.005 " to 0.015 " for a part with 12 " in diameter and 15 " in height. The layer thickness is between 0.003 and 0.02 " [2]. 


\section{Ballistic Particle Manufacturing (BPM)}

The ballistic system uses a piezo-driven inkjet mechanism to shoot droplets of melted materials, which weld together, onto a previously deposited layer (Fig 3). A layer is created by moving the droplet nozzle in $\mathrm{x}$ and $\mathrm{y}$ directions. After a layer is formed, the base plate lowers a specified distance and a new layer is created on the top of the previous one. The overall accuracy is plus or minus 0.004 ". The layer thickness is about $0.0035 "[2]$.

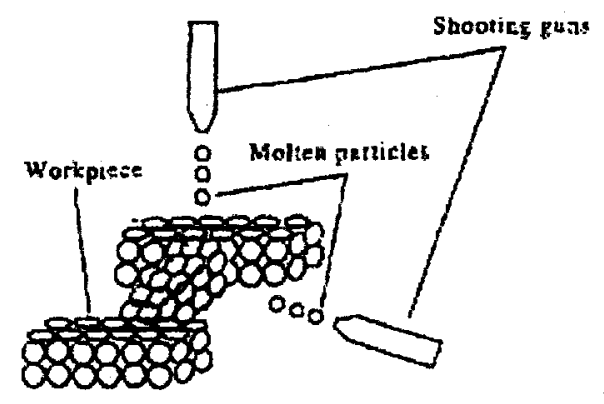

Fig 3: BPM Schematic [2]

Three-Dimensional Printing (3D Printing)

In the 3D Printing process (Fig 4), a layer of powder is spread on the top of the piston
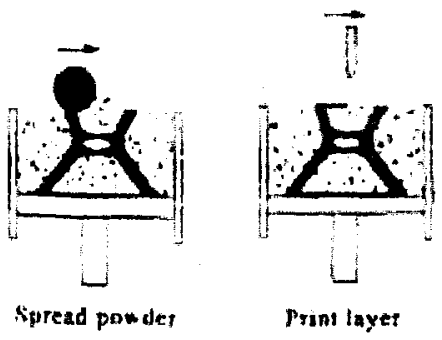

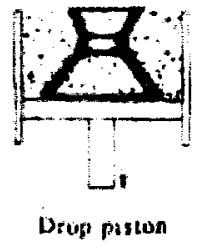

Fig 4: 3D Printing Schematic [2] in a cylinder. An inkjet printing head then projects droplets of binder material onto the powder at the place where the solidification is required according to the information from the computer model. After one layer is completed, the piston drops a predefined distance and a new layer of powder is spread out and selectively glued. When the whole part is completed, head treatment is required to 
enhance the bonding of the glued powder, and then the unbounded powder is removed. The overall accuracy has been tested at $+/-0.013$ " [3]. It has also been reported that the layer thickness varies between 0.003 " to 0.010 " and the minimum feature size is 0.017 ".

\section{Laminated Object Manufacturing (LOM)}

The LOM processes produce parts from bonded paper plastic, metal or composite

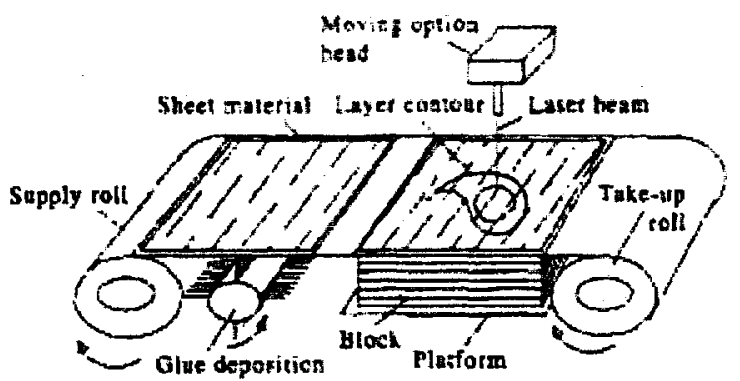

Fig 5: LOM Diagram [2] sheet stock. LOM machines bond a layer of sheet material to a stack of previously formed laminations, and then a laser beam follows the contour part of a cross-section generated by CAD to cut it to the required shape, as can be seen in Figure 5. The layers can be glued or welded together. The excess material of every sheet is either removed by vacuum suction or it remains as the next layer's support. The models can be constructed with an accuracy of $+/-0.005 "$ and do not shrink or distort because of the use of sheet material. The thickness of a layer is between $0.002 "$ and 0.02 ". Furthermore, the materials used to make the prototypes are less fragile than those made from photopolymers [2]. Table 1 summarizes the different RP technologies and their characteristics with respect to layer thickness and accuracy. 
Table 1: RP Technology Comparison

\begin{tabular}{|c|c|c|}
\hline RP Technology & Layer Thickness Range & $\begin{array}{c}\text { Accuracy } \\
\text { (Tolerance per linear inch) }\end{array}$ \\
\hline SLA & $0.004 "-0.030 "$ & $\begin{array}{c}0.1 \%-0.5 \% \text { of overall } \\
\text { dimension }\end{array}$ \\
\hline FDM & $0.010 "-0.250 "$ & $0.001 "-0.050 "$ \\
\hline SLS & $0.003 "-0.020 "$ & $0.005 "-0.015 "$ \\
\hline BPM & $0.003 "$ & $+/-0.004 "$ \\
\hline 3D Printing & $0.003 "-0.010 "$ & $+/-0.013 "$ \\
\hline LOM & $0.002 "-0.020 "$ & $+/-0.005 "$ \\
\hline
\end{tabular}

The merits of rapid prototyping lie in the fact that it can produce a prototype very early in the product development cycle, even before tooling, and enhance the design process by providing rapid and effective feedback to the designer for visualization and confirmation. Rapid prototyping can dramatically shrink the time and expense required to bring a new product from initial thought to production.

Although the current RP process overcomes many limitations of traditional manufacturing, the desire to use rapid prototyping for short-run manufacturing is continuously urging development of a cost-effective technique that will produce completely finished quality parts in a very short time. To meet the new demands of industry, rapid prototyping must be improved in the aspects of production speed, accuracy, and cost. Thus, a new HRP system was proposed to improve the aspects listed above through performing both deposition and machining in a single station. This combination of extrusion and material removal will allow the machine to produce high quality finished parts. Thus, the new HRP system can dramatically reduce the total build time and improve the shape accuracy compared with the conventional RP systems. In addition, this new system can be used to retrofit conventional RP systems, so that their degree of accuracy is also increased. 
In this report, a newly developed rapid prototyping process based on the hybrid technique, i.e. additive and subtractive process, and its hardware framework are presented. In addition, a variety of Visual basic 6 programs based on simple geometric shapes are also provided in order to assist the further reduction of the total build time for prototype parts.

\subsection{Other Hybrid Methods}

The objective of an HRP System is to reduce development time for a new product to enter the market. Junghoon Hur [4], developed a HRP system, called ECLIPSE-RP. The

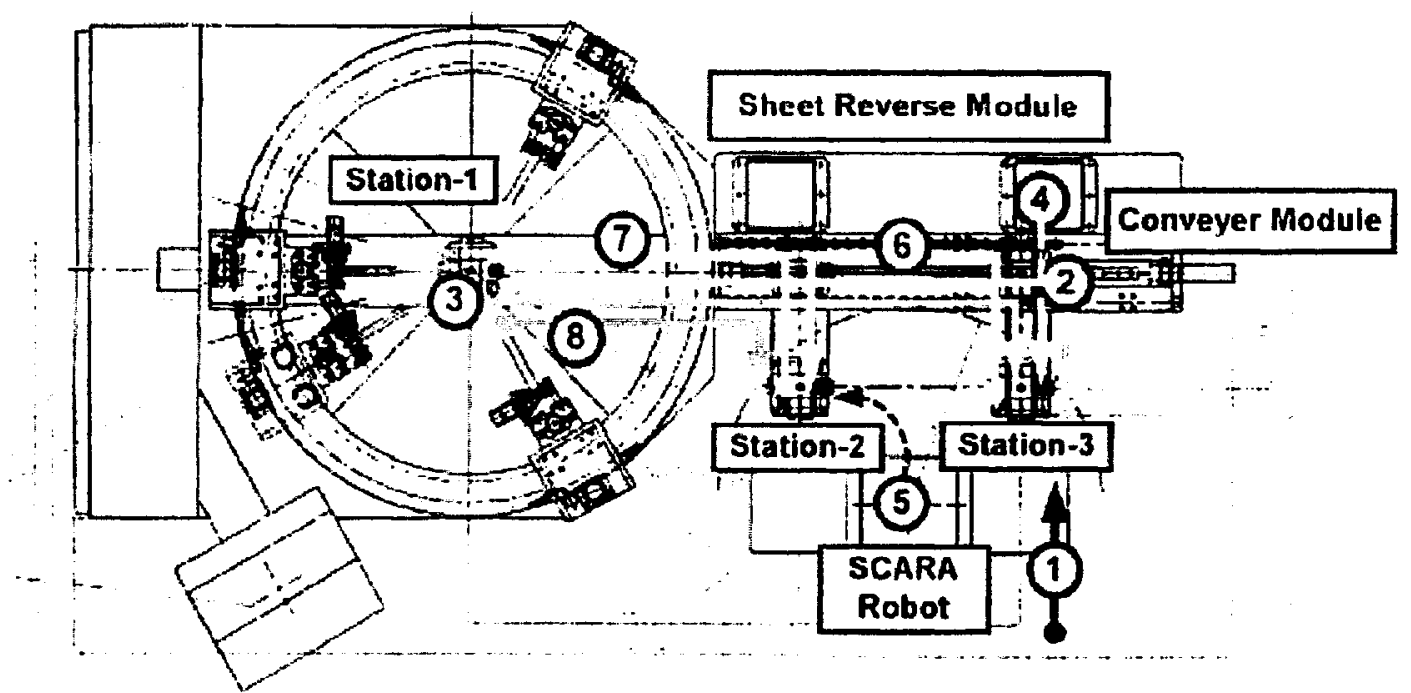

Fig 6: ECLIPSE-RP Diagram [4]

hardware for this system consisted of six sub-modules: Station-1, Station-2, Station-3, Conveyer module, Set-up reverse module and the Adhesive dispense module, as

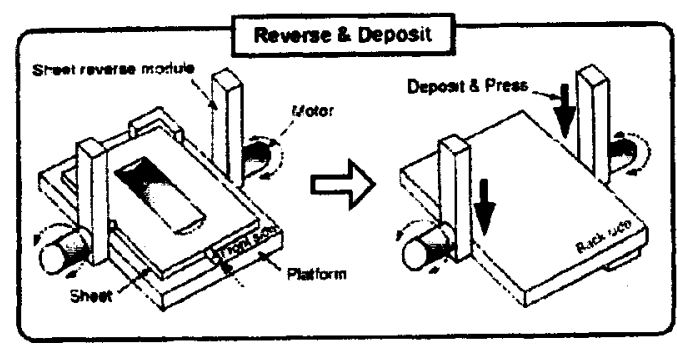
shown in Figure 6. Station-1 was used for all the machining processes. It consisted of a 6-axis parallel mechanism, which enables 
5-axis simultaneous machining, 5-sided prismatic machining, and vertical turning all in a single step. Station-2 was used as the location where new Deposition Feature Segments (DFS) were continuously deposited until the whole building process finished. Station-3 was used for new sheet materials and where the main process cycle begins. The conveyer module automated the work piece to flow between the stations while the set-up reverse module was used for inverting the sheet material whose backside has been completely machined (Fig 7). Finally, the adhesive dispense module ejected the cyanoacrylate on the top of the already-deposited DFS's along the predefined bonding paths. Furthermore, Hur's hybrid RP system uses very thick chemical wood sheets as building materials, compared with conventional RP systems, which use thinner layers. Through his research, Junghoon Hur found that this new hybrid system could effectively solve the limitations of the conventional manufacturing process through its unique hybrid process and process planning system. Because the proposed hybrid process performs both deposition and machining with a facility for inverting set-up direction, it is expected to become a new cost-effective manufacturing technique that will produce completely finished quality parts in a very short time for any shape. However, one important note to mention here is that this hybrid RP system first deposits all the layers together and then it systematically begins the machining process. 


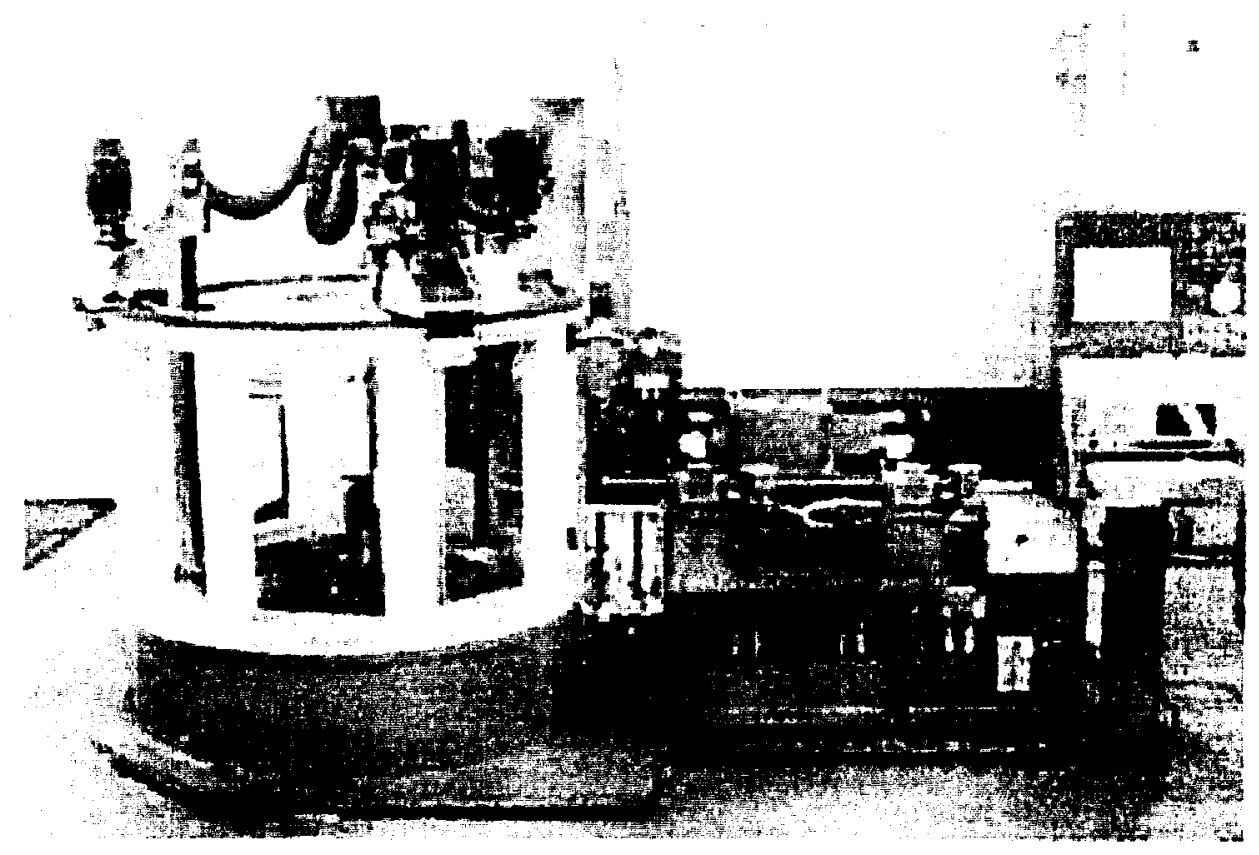

Fig 8: ECLIPSE-RP Photograph [4]

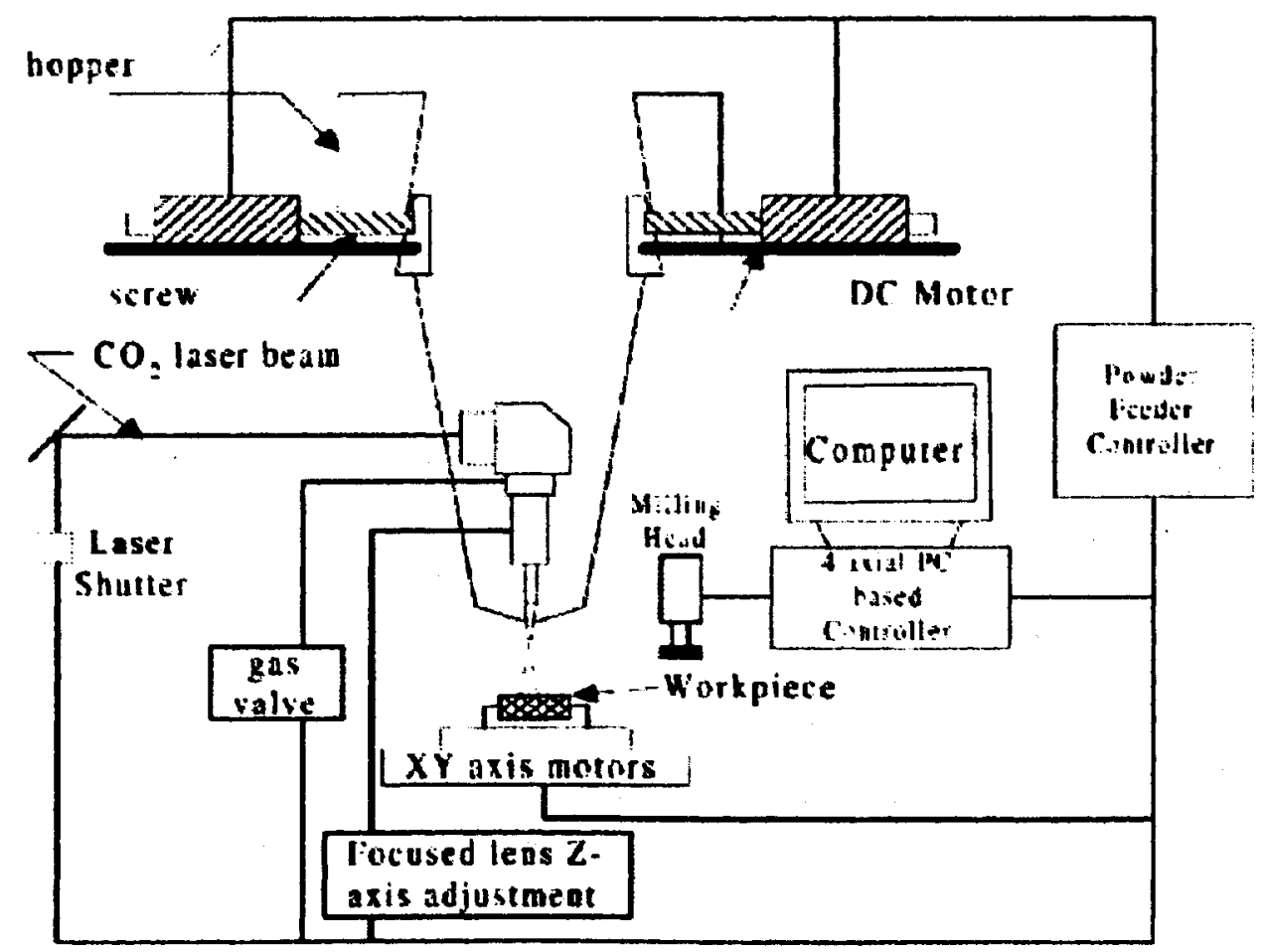

Fig 9: Mould Fabrication Using SLS [5] 
A different hybrid system was proposed by Jeng-Ywan Jeng and Ming-Ching Lin [5]. These authors researched a hybrid process to facilitate mould fabrication by using selective laser cladding and milling. Their design included a Rofin Sinar RS820 1500W $\mathrm{CO}_{2}$ laser that was employed in the SLS system as the power resource for the laser cladding process. An axial PC-based controller was used to position the work piece on the $\mathrm{X}-\mathrm{Y}$ table. The control software moved the work piece to the desired position and started the cladding operation; the latter operation included the movement of the work piece, and the control of the powder feeders, the laser shutter, and the gas-shielding valve. Once the cladding operation was completed, the focused lens of the laser was moved up and the work piece was moved to the position beneath the milling head. Then, the surface of the work piece with the clad profile was machined to achieve the desired accuracy and produce a smooth surface, ready for the next cladding operation. The authors concluded that mould fabrication modified by the hybrid processes of SLS and milling is feasible if the layer thickness can be further reduced.

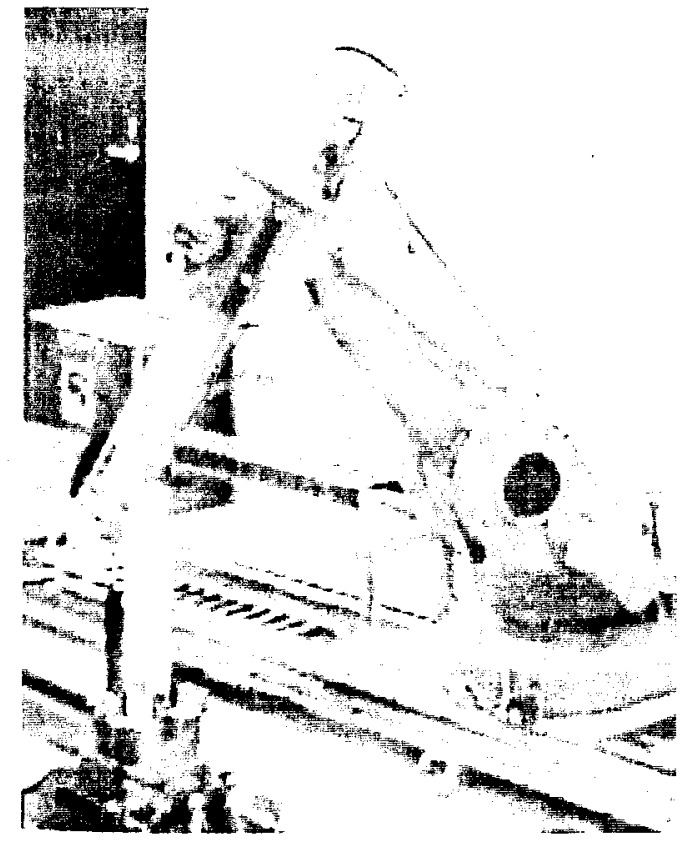

Fig 10: Robot Machining [6]
Authors Y.H. Chen and Y. Song [6] also developed a hybrid RP system. The authors ventured that although robot machining has its unique features, it also has the same inherent problems of $\mathrm{CNC}$ machining methods. The most obvious problem is that models with hollow features or deep cavities cannot be machined because when the tool tries to 
machine a bottom point inside a cavity, collision occurs between the tool holder and the model surface. To solve this problem, a layer based machining method was proposed. First, the whole model is sliced into layers as in most RP systems, and then the lower layer is machined first, since the cavity on this layer is much shallower than that of the original model. As a result, the chances of a collision occurring between the tool and the tool holder are reduced. After the machining of the lower layer, another layer is glued to it with adhesive. Then the machining of a new layer can be repeated. The authors concluded that their current system is capable of machining large-scale models with simple hollow features. Furthermore, consecutive machining on each layer produces better results and is more practical than machining the model after deposition is complete.

\subsection{Proposed Hybrid Design}

The Hybrid RP system (HRP system) presented in this report was constructed from the modification of an existing machine and the incorporation of a machining spindle similar to the cited researched papers. The HRP system head consists of a material extrusion head and a milling head. The material extrusion head initially deposits the first layer of plastic or wax onto the X-Y table. A quarter of an inch (1/4") end mill is then used to machine around the perimeter of the deposited layer as well as the top surface of the layer. Once this operation is complete, a new layer is deposited and subsequent machining follows. The HRP system is considerably small when compared to the RP systems that were researched by the author. The working area of this system is no larger than 4" x 4". 
A program (DeskNC) that allows the input of G-codes is used to move the X.Y table through the driver board, as shown in Figure 11. However, to generate the appropriate G-

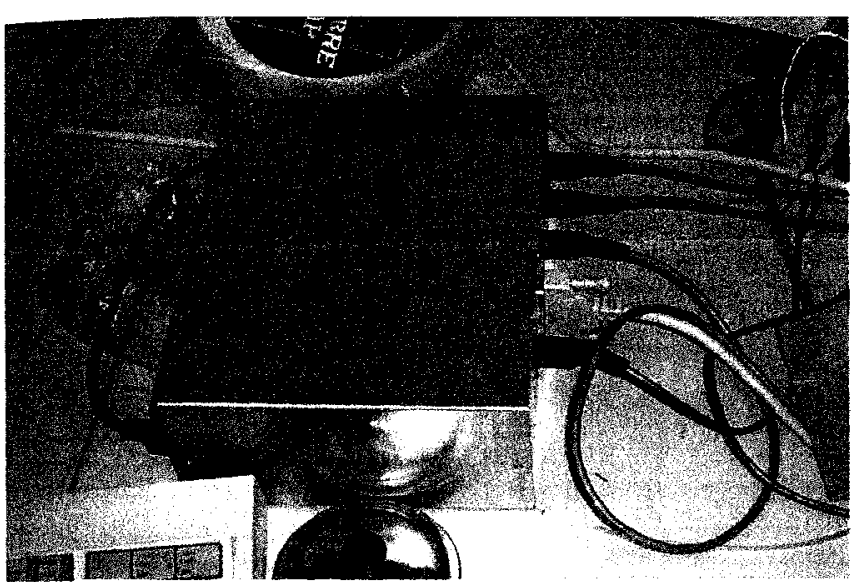

Fig 11: I/O Board For HRP codes for a given shape would have required hours of tedious manual input for the designated coordinates. To simplify this situation, a Visual Basic 6 program was written to produce the necessary G-codes to produce simple geometric shapes.

In this work, the material extrusion head and the end mill are mounted on the same X$\mathrm{Y}$ table with the same degree of movement, thus creating a true single station deposition and layered machining system. This is different than in previous work as discussed in Section 1.2. The advantage in this system is that there is no need for a transferring mechanism, such as a conveyer system, to transport the part from one station to another where vibrations in the movement could reposition the part and thus give inaccurate results. A user interface was created using Visual Basic 6 to allow the user to input any dimension on a given number of shapes he/she wishes to produce with the HRP system. The program then produces a G-code program to the users specifications. These codes are then passed along to the RP system, permitting the additive production of plastic or wax modelling to begin. The G-code also takes into account the movements of both the material extrusion head the end-milling head. The G-codes were executed on a version of DeskNC, which, graphically displayed, in real-time, the movement of the milling and the extrusion heads. Furthermore, the $\mathrm{X}, \mathrm{Y}$ and $\mathrm{Z}$ coordinates of both heads will be 
continuously displayed while the machine is running. An unexpected final advantage of this system was its size with respect to portability and mobility. The HRP system can quickly be disconnected and reconnected at another location with a minimal amount of time.

It is crucial to mention that the apparatus in this experiment is not comparable to the equipment mentioned in Section 1.2. Complex shapes with deep cavities on both sides are not possible with this 3-D hybrid machine. The intent is to show that this proposed system has the capability to produce prototype parts with a satisfactory accuracy scale. Furthermore, this system will demonstrate the ability to retrofit an existing machine into a hybrid system. The accuracy proposed would become apparent due to the surface machining that is performed to reduce the "stair-step" inaccuracies that are seen in other rapid prototyping systems. 


\section{CHAPTER 2 - EXPERIMENTAL APPARATUS AND PROCEDURE}

\subsection{Previous Rapid Prototyping Apparatus}

The current RP machine (figures 12 and 13) located in the T-building at Ryerson University was originally designed by Hassan Hamzeh in 2000 as a fourth year

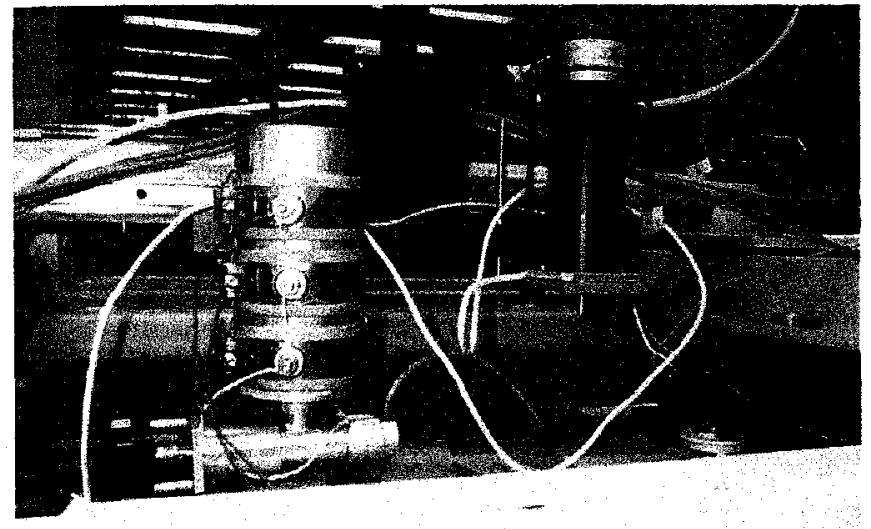

Fig 12: Front View of RP Machine graduating thesis project. The $\mathrm{RP}$ system resembles that of a bridge design used in most CNC punch machines. The working table is fixed while the wax deposition head is allowed to move in the $\mathrm{X}$ and $\mathrm{Y}$ directions. Linear ball railings allow

for the smooth motion in the axes mentioned. Stepper motors are used to slide the bridge over the ball railings and to ensure an accurate method of motion. The paraffin wax was to be stored in a 3-inch diameter aluminium cylinder (see figure 14) with a wall thickness of a quarter of an inch. The height of this cylinder is 6 inches and there are three heating coils, which encompass its exterior surface. Mr. Hamzeh also had designed an adjustable working table to accommodate the $\mathrm{Z}$-axis but was unable to assemble it before the submission of his paper. The schematics of the RP design are available in Appendix 1.

The method of wax deposition was originally thought to have been more of a continuous output

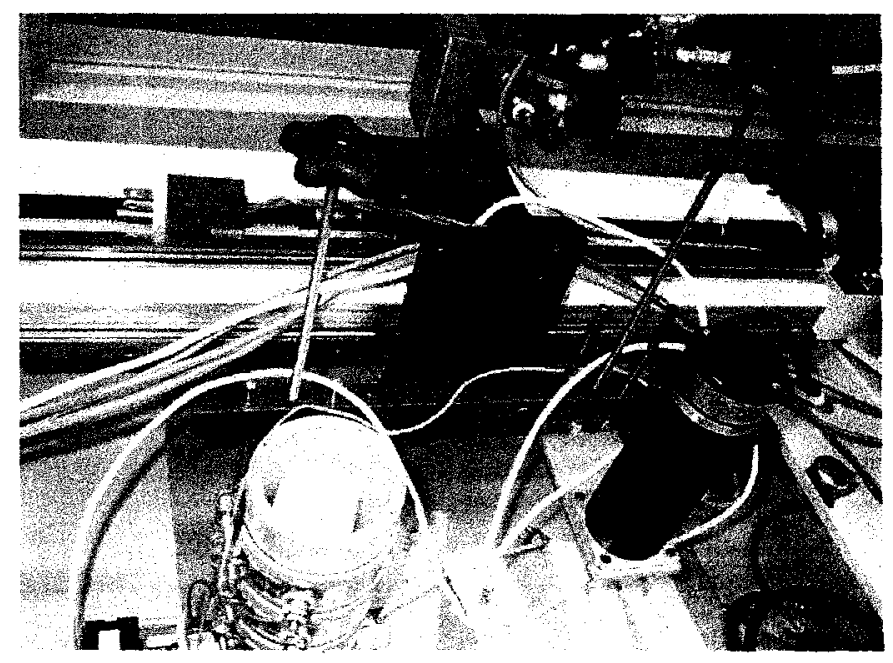

Fig 13: Top View Of RP Machine 
rather than individual droplets in close proximity. Mr. Hamzeh had designed an aluminium cylindrical holder to allow a given amount of solid wax (fig 15) to be placed inside. This container was then wrapped with heating coils and was connected to a variable temperature controller box. However, his research stopped short of designing and manufacturing an appropriate nozzle for the exodus of the wax. In the summer of 2001, a research student by the name of Sal Lamberti designed and manufactured a Fused Deposition Model (FDM) nozzle. This researcher went through several design iterations until the single screw pump was chosen. Mr. Lamberti reported that this design provided the necessary means of metering out the wax accurately, and also provided the simplest machining design. He outlines that his main concern was that the nozzle should have the ability to break the surface tension of the wax in order to allow exportation.

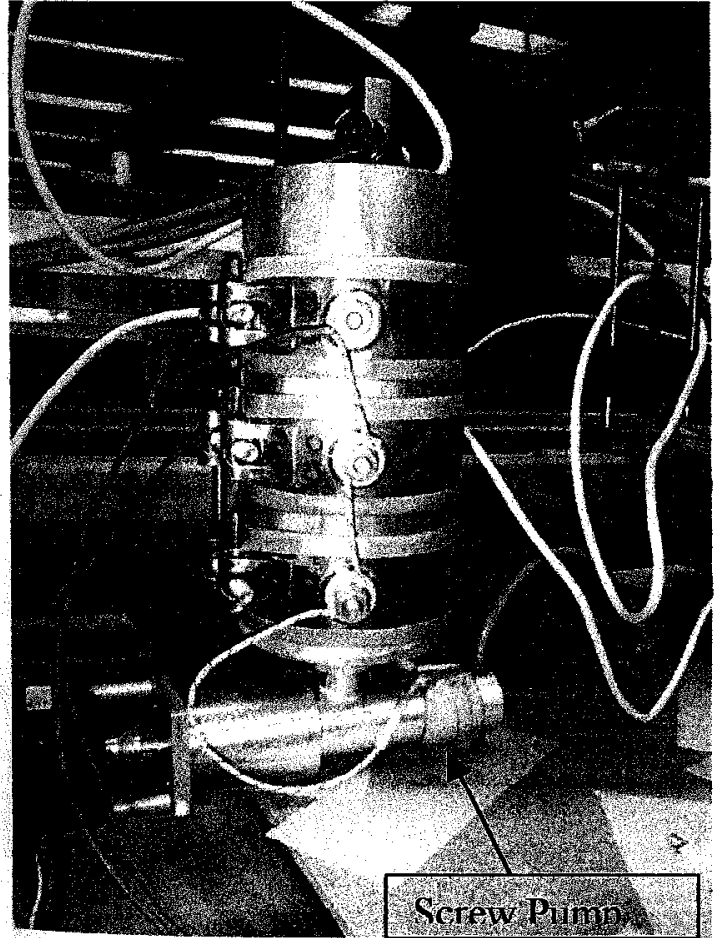

Fig 14: Molten Wax Reservoir With Screw Pump

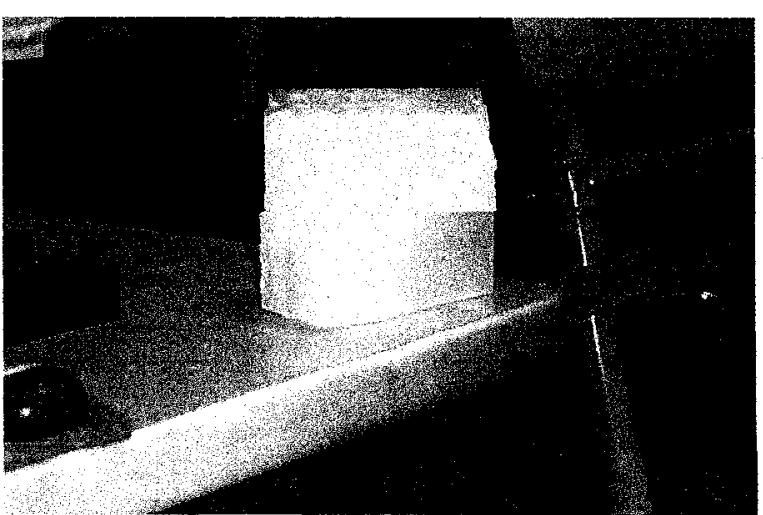

Fig 15: Paraffin Wax 


\subsection{Hybrid Rapid Prototyping Design I}

Although the last two researchers did a tremendous job on this RP system, it was left somewhat incomplete. The initial goal of this research was to complete, calibrate and modify the existing RP system into a Hybrid Rapid Prototyping (HRP) system. The modification process started with the following issues being addressed.

The first issue to be addressed was the addition of a machine-head. A DC-motor (fig 16) was mounted on the bridge beam to be used as the spindle for the machining head. The back supports of the Z-axis table were removed and realigned (as shown in Figure 17) in order to level the aluminium-working table. A custom-made brass collet (fig 18) was designed and manufactured to securely hold the $1 / 4$ " end-mill into place. On the molten wax reservoir, with the heating coils wrapped around the basin and no

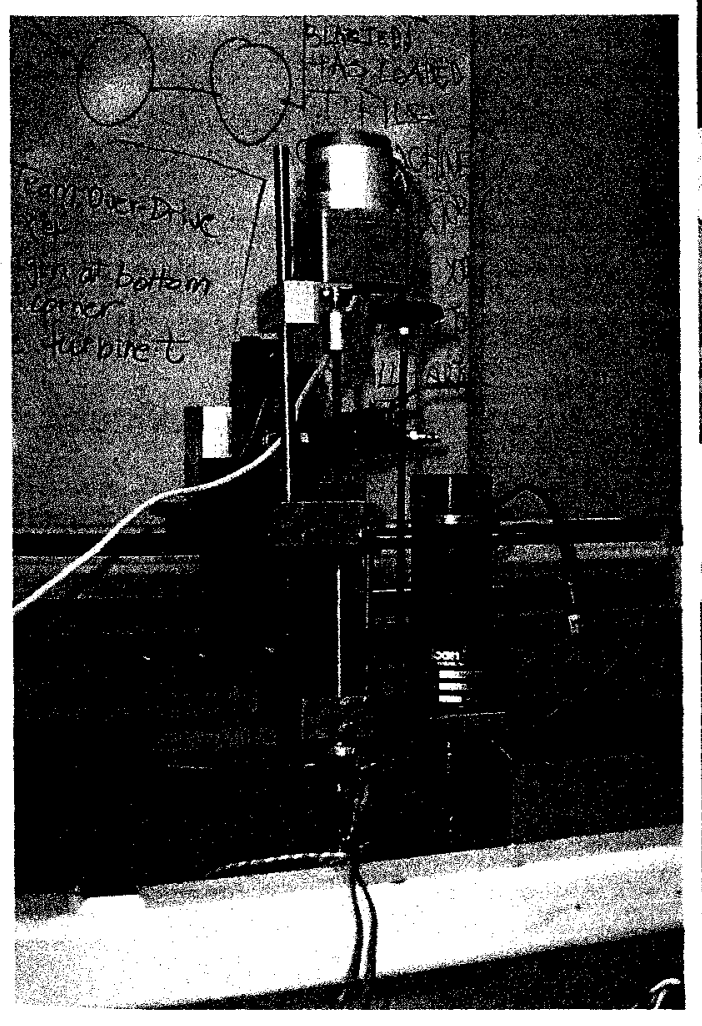

Fig 16: Z-axis DC Motor

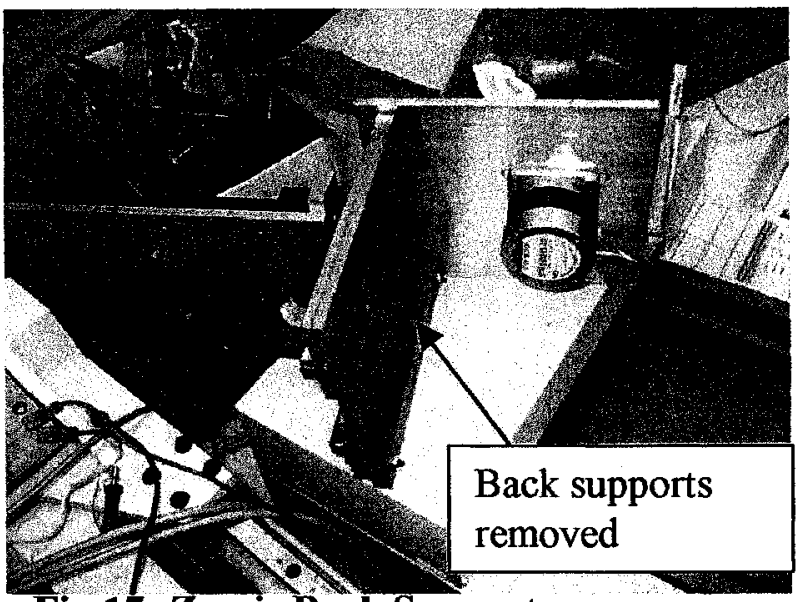

Fig 17: Z-axis Back Supports

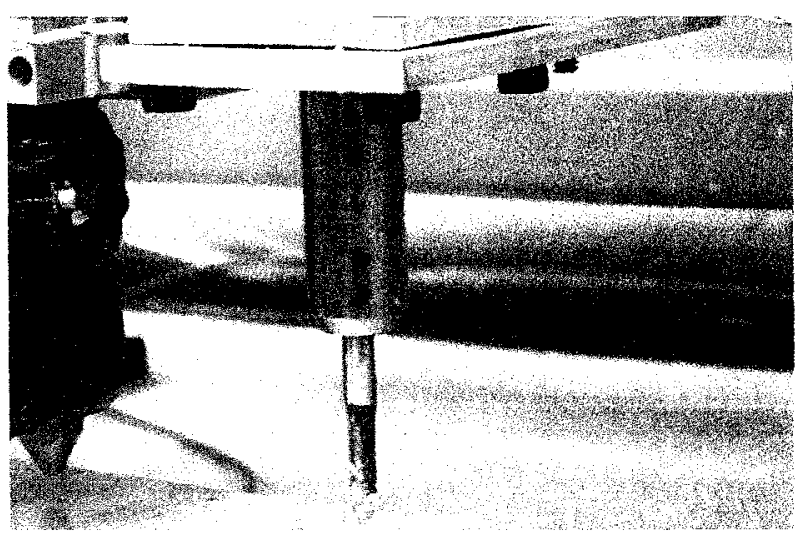

Fig 18: Custom Brass Collet 
protective cover designed, this presented a very dangerous electrical hazard. To overcome this problem, a stainless steel/plexi cover, shown in figure 19, was designed and manufactured to protect the user from the open electrical wires and the intense heat that would be generated from the melting of the wax. A clear plexi cover was used in front of the safety guard to still allow sightseeing of the basin.

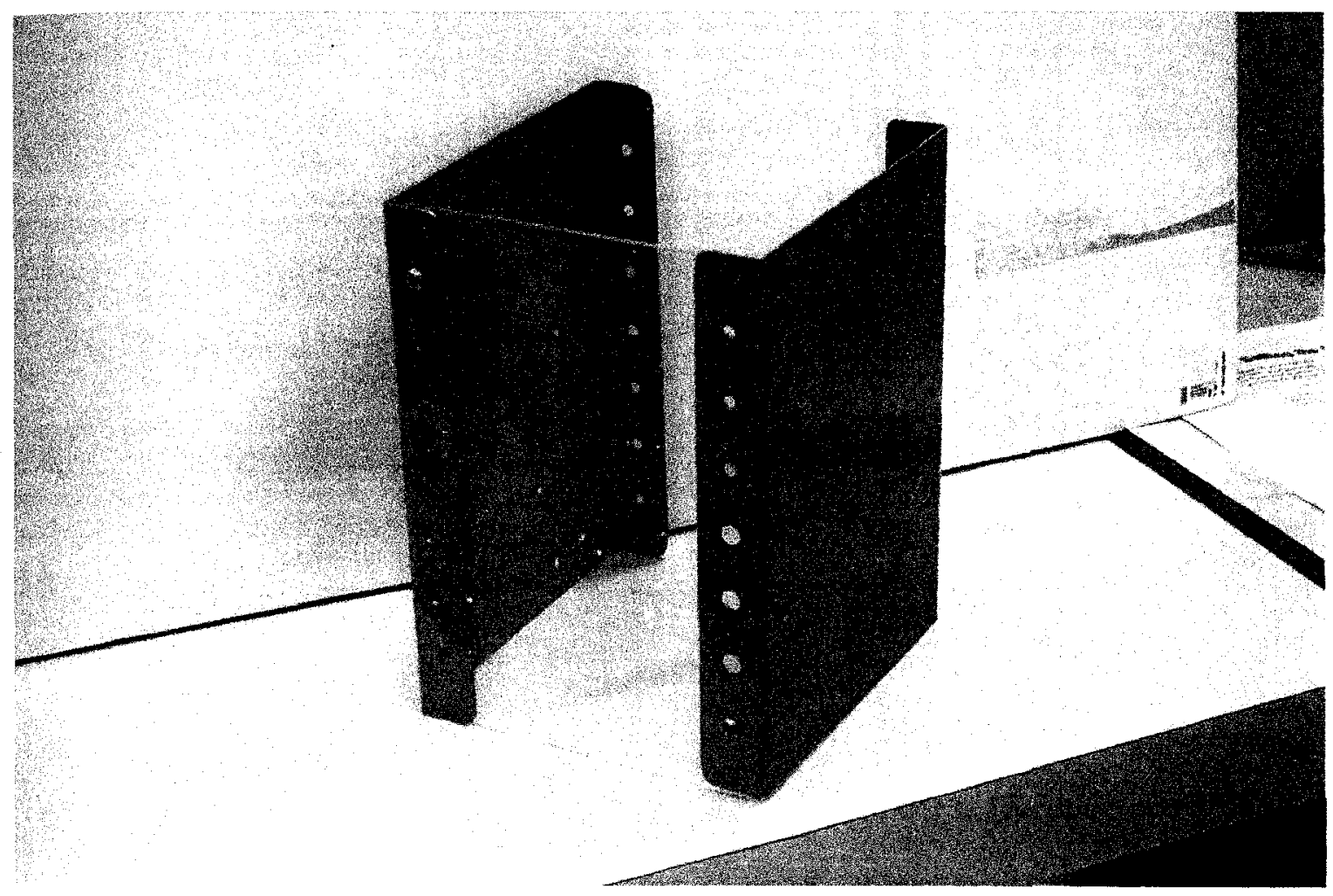

Fig19: Custom Stainless Steel Protective Cover

Furthermore, the single screw design needed to be slightly altered. The clearance between the lead screw and the aluminium piston was slightly too large. This resulted in the wax leaking out between the clearance and solidifying on the outside. This solidification hindered the performance of the single screw. To combat this problem, a much more intimate fit was designed between the screw and the piston. This new configuration resulted in a clearance of 0.002 " between the piston and the cylinder. Furthermore, a higher torque stepper motor was used to overcome the possible 
solidification of the wax if it occurred. The previous stepper motor (fig 20) accompanied by a custom built variable speed controller box (fig 21) was not strong enough to achieve this task and was replaced with the higher torque motor shown in figure 22.

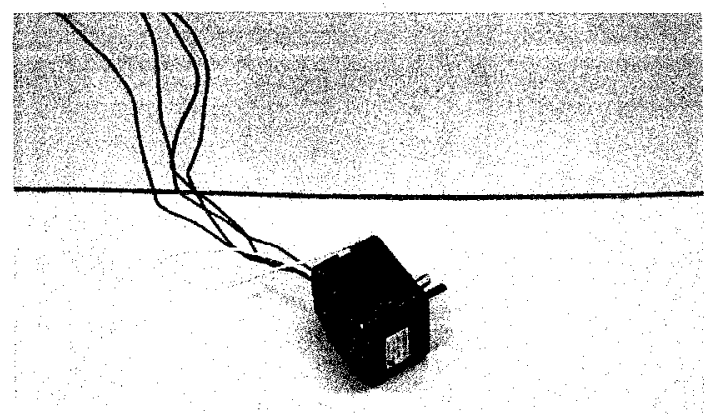

Fig 20: Original Pump Motor

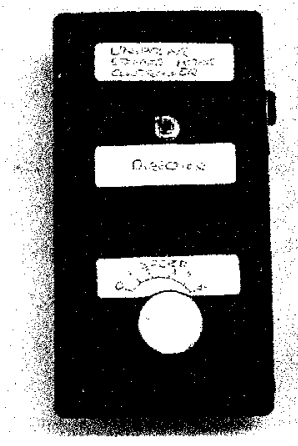

Fig 21: Original Speed Variable Control Box For Stepper Motor

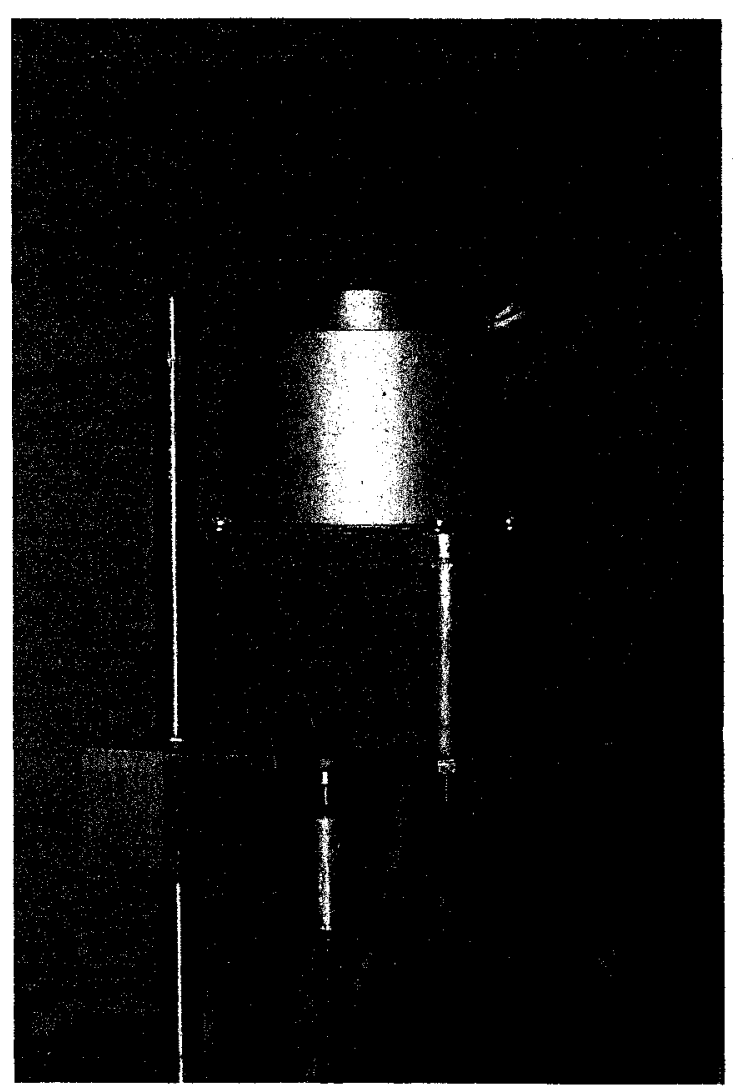

Fig 22: High Torque Replacement Motor

\subsubsection{HRP Design I Results}

After many months of calibration and testing, the first design was abandoned. The wax became too unpredictable to control due to its low viscosity. The wax continuously dripped and leaked out of every pore of the molten wax reservoir and screw pump. Although the single screw was removed and re-machined for a much tighter tolerance, it still did not stop the leakage of the wax. The stepper motor was again replaced to allow 


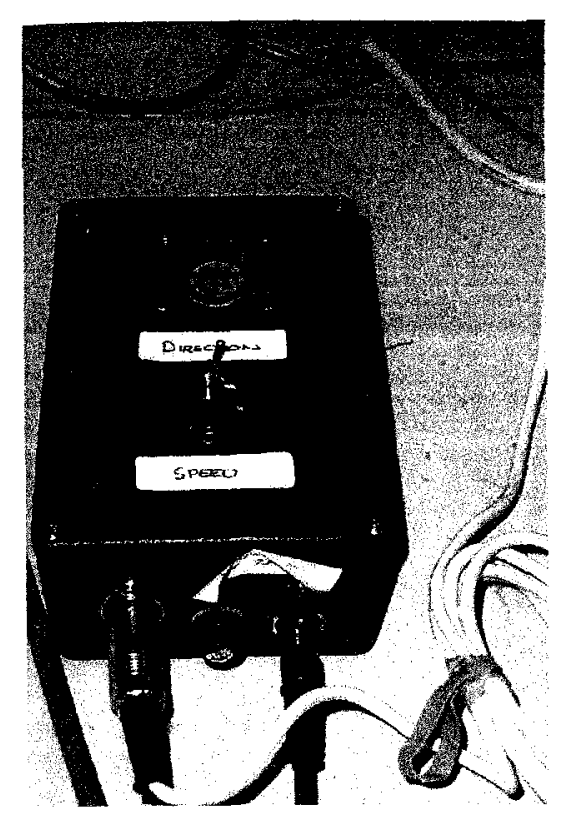

more torque to be provided to the screw pump action with no noticeable improvements. A more powerful variable speed control box for the stepper motor was again designed and manufactured (as shown in Fig 23) in order to control the metering of the wax from the nozzle. This second variable adjuster was needed to compensate the stronger stepper motor being used. However, the leaking of the wax could not be controlled and as a result the design was aborted.

Fig 23: Second Speed Variable Control Box for the Stepper Motor

\subsection{Hybrid Rapid Prototyping Design II}

Since the previous work was abandoned, a totally new material delivering system was

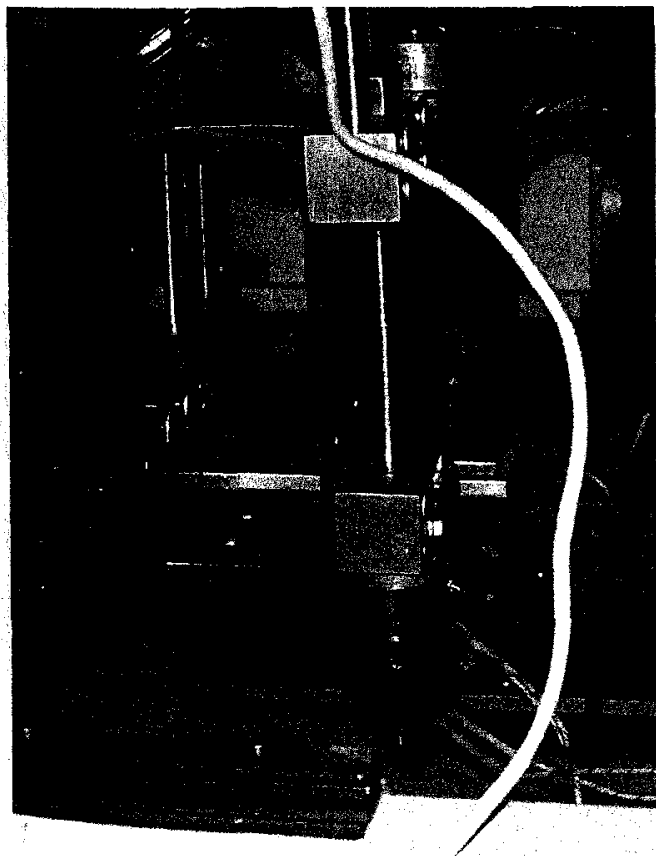

designed which was based on extrusion rather than liquid deposition. Polystyrene plastic was deposited into the cylinder. A piston connected to a lead-screw being driven by a stepper motor would then meter out the heated plastic on the working table. Thus the material would be extruded continuously rather than droplet depositing. A photograph is shown in Fig 24 while full AutoCAD drawings and a 3-D rendering of the design are available in Appendix 2. The

Fig 24: New Wax Extrusion System 
working table was then assembled into place to accommodate both the plastic extrusion head and the end-milling head.

\subsubsection{HRP Design II Results}

During the first experiment a huge obstacle presented itself. The Polysperene plastic has a melting temperature of $240^{\circ} \mathrm{C}$. However, the heating control box and the heating

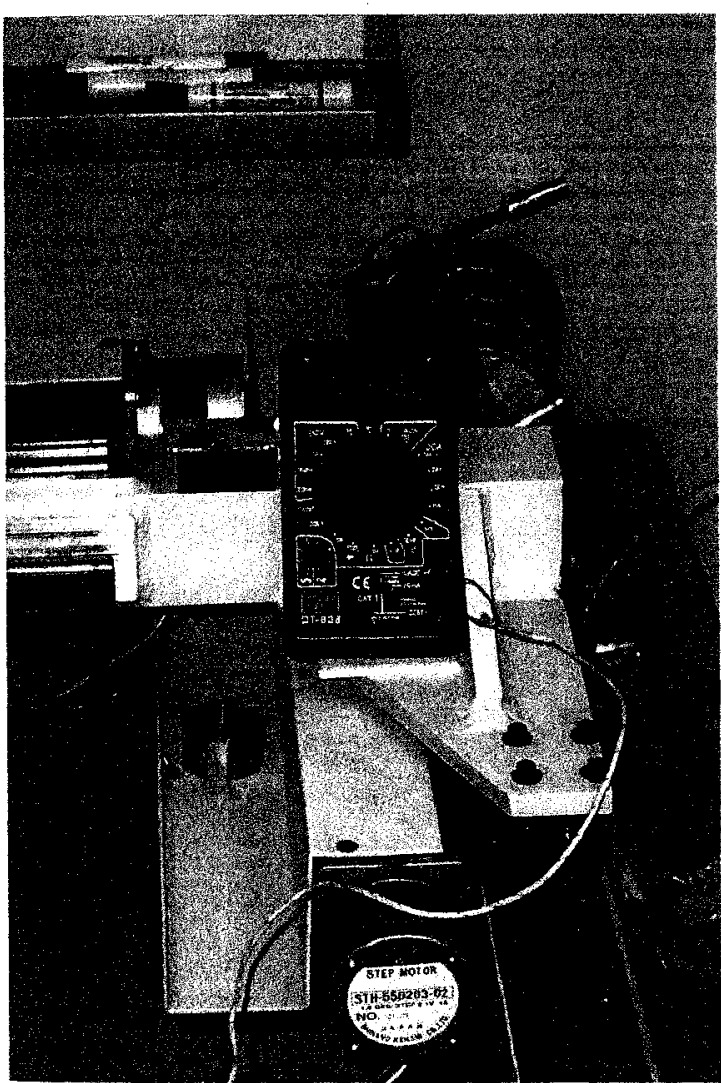

Fig 25: Digital Temperature Display

coil being used only raised the cylindrical temperature to $202^{\circ} \mathrm{C}$. The temperature was measured from a probe connected directly to the nozzle portion of the cylinder and was displayed on an electrical temperature reader (see figure 25). Furthermore, because the melting temperature could not be reached, once the plastic was extruded out with great force, it almost immediately solidified into a very hardened state. The remaining plastic inside the cylinder had also solidified once the heating coils were de-energized. The damage was tremendous. There was no way to get the residue plastic out unless the melting temperature was reached. And the current heating mechanism could not reach the melting temperature. A hammer and a chisel were used to break the plastic and then an oxy-acetylene torch was used to melt the plastic. The plastic was finally removed but the damaged caused to the cylinder was irreparable. The cylinder was cracked and the threaded holes used for supporting the cylinder were stripped. 


\subsection{Hybrid Rapid Prototyping Design III}

The previous cylinder for the second design iteration was constructed out of aluminium while the piston was manufactured from steel. Their clearance was set to 0.002 " in order to accommodate the expansion of both materials when induced with heat. Since the coefficient of expansion of aluminium is greater than steel, the tolerance between the piston and cylinder should increase allowing for movement between the piston and the cylinder. However, as mentioned the aluminium was not strong enough to withstand the Polysperene plastic removal process and had to be replaced. Furthermore, the expansion of the aluminium could not always be accurately predicted. In this third attempt, the cylinder was refashioned from steel to provide much more needed strength for the cylinder and also to match the expansion coefficient of the piston. Therefore, both components would have the same expansion and contraction characteristics. Also, the Polysperene plastic was discarded due to the fact that the melting temperature could not be reached and thus the paraffin wax was revisited.

Initially, the wax was broken up and placed inside the new cylinder a piece at a time. The piston was used to compact the wax and then extruded. This process was very tedious, time consuming and proved to be flawed. When the piston extruded the wax out, there were air pockets between the wax and a continuous deposition could not be possible. To avoid this problem, the wax was melted by a hot air gun (fig 26) in a steel pot (figure 27) and was then poured into the cylinder to cure. This created, a non-porous slug of wax that could be uniformly heated and extruded. After an initial trial and error period, the third attempt described above was found to be the most suitable to carry on 
experimentation with the geometric shape program phase previously written in Visual basic 6 .

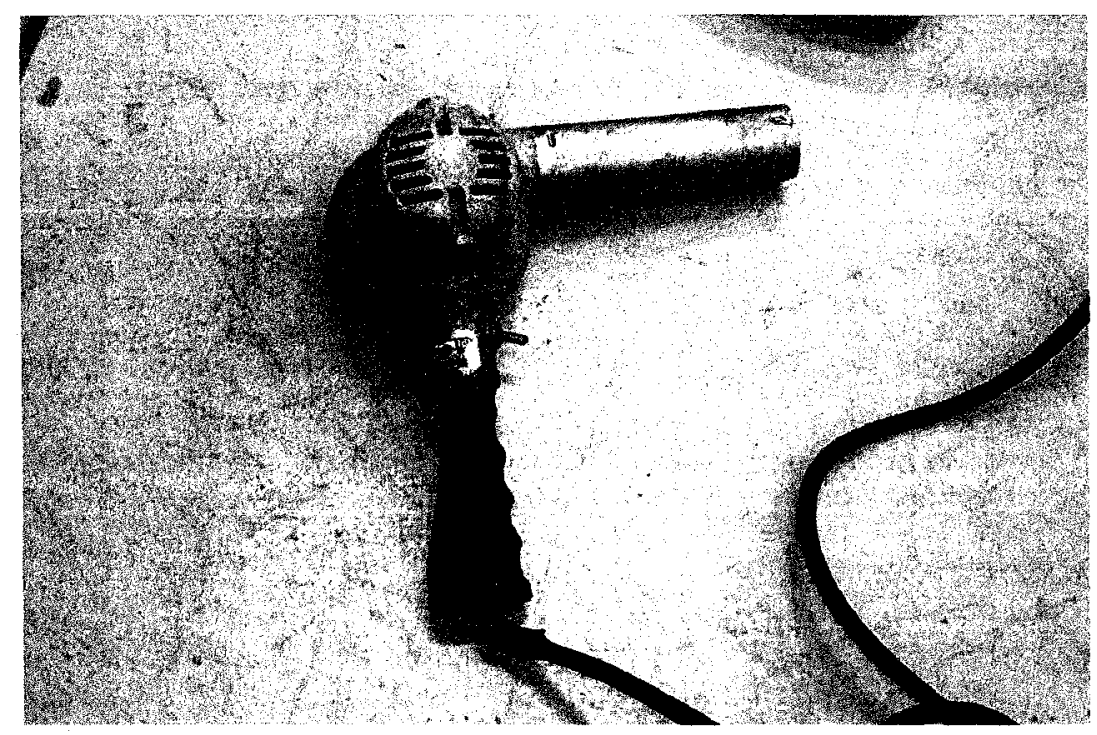

Fig 26: Hot Air Gun

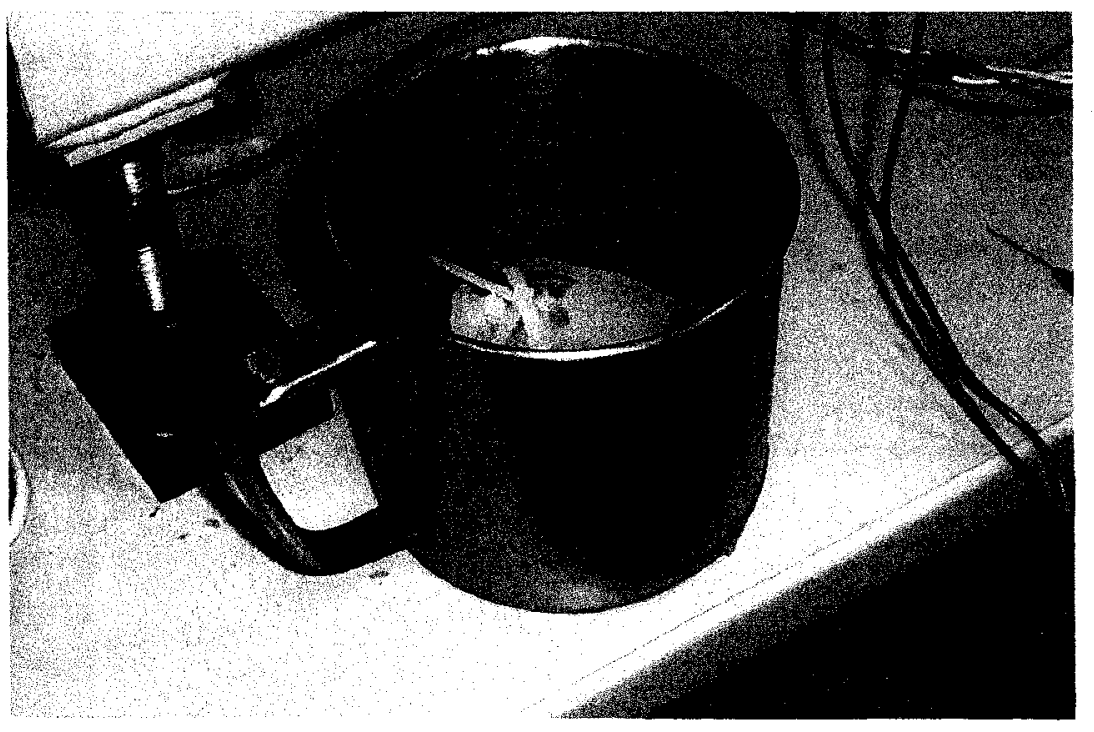

Fig 27: Steel Pot Used For Melting Wax 


\subsection{Testing Temperature, Gap size, Feed-rate and Pump-rate}

\subsubsection{Phase 1}

The next step involved finding an optimum temperature for the extrusion of the wax, an adequate gap size between the nozzle and the working table to allow the wax to stick on the working table before solidifying and an appropriate feed-rate for the machine and pump-rate for the piston. An approach was taken to find the variables mentioned above. Table 2 illustrates a variety of temperatures, gap sizes, feed-rates and pump-rates that were tested for peak performance. The correct procedure for optimizing such variables is to design an experiment based on the rules of statistical analysis and then to use methods of calculating the roundness of cylinders and the squareness of cubes (CMM-Coordinate Measuring Machine) in order to correctly identify how these variables interact with each other. Furthermore, statistical tests will be needed to determine the randomness of the values in order to validate their importance. The magnitude and depth of such an undertaking was beyond the scope of this project. In order to gain some raw preliminary data, a subjective criteria based on the author's experience was created to isolate optimum variable conditions with respect to initial adhesion qualities, uniform distribution of the wax, and suppressing an overflow of the wax.

These three criteria were deemed to be the most important factors affecting the out come of the prototypes. Initial adhesion refers to the instant when the paraffin wax makes contact with the working surface. Referring to figure 28 , it is clear that for temperatures between $49^{\circ} \mathrm{C}$ and $52^{\circ} \mathrm{C}$, the scores associated with initial adhesion were extremely low. This was due in part to the solid state of the wax during those temperatures and the lack of an adhesive ability on the part of the working table. These 
Maximum Score Per Temperature vs. Temperature (0.125" Gap, 1/8" Nozzle Diameter)

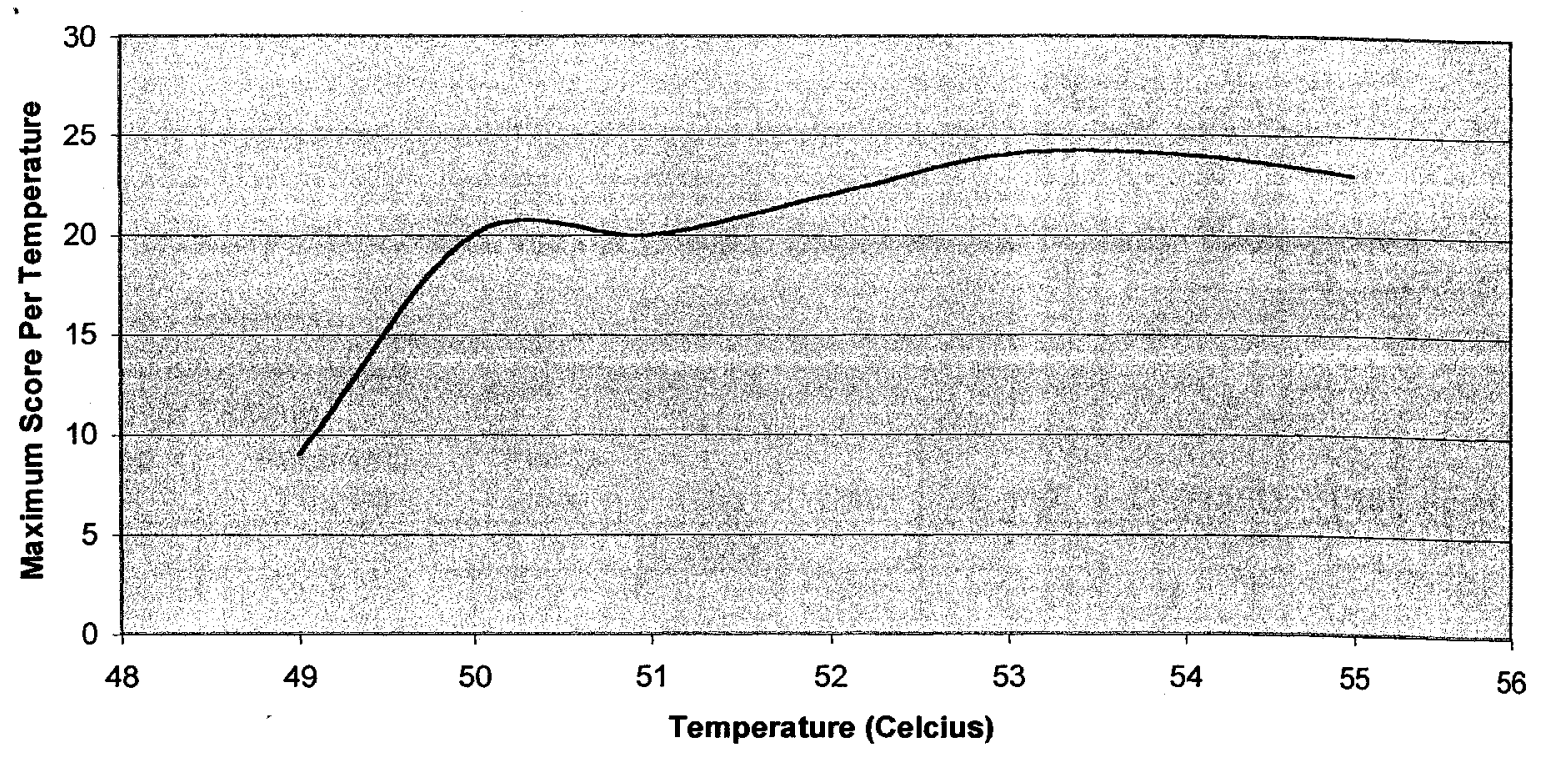

Fig 28: Max Score/Temperature vs. Temperature (1/8" gap, 1/8” Dia.)

two factors combined inhibited the bonding of the wax to the surface. The second criteria (uniform distribution) dealt with a constant width of wax being extruded over a predetermined distance. This criterion was deemed to be extremely important because the program written for the production of simple geometric shapes was heavily dependent on a known and fixed width. The final criterion, which dealt with an overflow of material, was used in order to systematically determine the optimum speed for the stepper motor and the optimum speed of travel for the HRP machine. Since the gap between the nozzle and the working table differentiated between $1 / 8$ " and $1 / 16$ ", suitable speeds had to be found in order to avoid an overflow of wax. Each criterion was given a weight out of ten marks and immediately after each trial; a numerical score was assigned to that specific configuration. 


\subsubsection{Phase 1 Results}

Initial testing from the lower temperatures, provided very poor results. The wax had obviously not reached its melting point and basically extruded out in solid form.

\section{Table 2: Score Sheet Based on 1/8" Nozzle Diameter and Metallic Table Surface}

\begin{tabular}{|c|c|c|c|c|c|c|c|c|}
\hline \multicolumn{9}{|c|}{$\begin{array}{l}\text { I.A. Initial Adhesion } \\
\text { U.D. Uniform Distribution } \\
\text { O.F. Over Flowing }\end{array}$} \\
\hline $\begin{array}{l}\text { Temp. } \\
\left.\text { ( }{ }^{\circ} \mathrm{C}\right)\end{array}$ & $\begin{array}{c}\text { Feed-rate } \\
\text { ("//min) }\end{array}$ & $\begin{array}{l}\text { Pump- } \\
\text { rate }\end{array}$ & Gap & Gap & I.A. (/10) & U.D. (110) & O.F. (/10) & Score \\
\hline \multirow[t]{3}{*}{49} & 3 & 1 & $0.0625^{\prime \prime}$ & & 2 & 3 & 4 & 9 \\
\hline & 3 & 2 & & $0.125^{\prime \prime}$ & 2 & 3 & 3 & 8 \\
\hline & 3 & 3 & & $0.125^{\prime \prime}$ & 3 & 3 & 3 & 9 \\
\hline \multirow[t]{5}{*}{50} & 3 & 2 & $0.0625^{\prime \prime}$ & & 3 & 4 & 3 & 10 \\
\hline & 4 & 2 & $0.0625^{\prime \prime}$ & & 4 & 4 & 3 & 11 \\
\hline & 4 & 3 & $0.0625^{\prime \prime}$ & & 4 & 6 & 5 & 15 \\
\hline & 3 & 2 & & $0.125^{\prime \prime}$ & 4 & 5 & 5 & 14 \\
\hline & 3 & 3 & & $0.125^{\prime \prime}$ & 4 & 5 & 7 & 16 \\
\hline \multirow[t]{8}{*}{51} & 1 & 1 & & $0.125^{\prime \prime}$ & 4 & 5 & 2 & 11 \\
\hline & 2 & 1 & & $0.125^{\prime \prime}$ & 2 & 2 & 2 & 6 \\
\hline & 3 & 1 & & $0.125^{\prime \prime}$ & 2 & 4 & 3 & 9 \\
\hline & 3 & 3 & & $0.125^{\prime \prime}$ & 4 & 5 & 4 & 13 \\
\hline & 3 & 3 & $0.0625 "$ & & 4 & 4 & 5 & 13 \\
\hline & 4 & 1 & $0.0625^{\prime \prime}$ & & 2 & 4 & 4 & 10 \\
\hline & 4 & 2 & $0.0625^{\prime \prime}$ & & 4 & 1 & 4 & 9 \\
\hline & 4 & 3 & $0.0625^{\prime \prime}$ & & 3 & 1 & 3 & 7 \\
\hline \multirow[t]{2}{*}{52} & 3 & 3 & $0.0625^{\prime \prime}$ & & 4 & 4 & 4 & 12 \\
\hline & 3 & 3 & & $0.125^{\prime \prime}$ & 6 & 8 & 8 & 22 \\
\hline \multirow{4}{*}{53} & 2 & 3 & & $0.125^{\prime \prime}$ & 7 & 7 & 7 & 21 \\
\hline & 3 & 2 & & $0.125^{\prime \prime}$ & 7 & 7 & 7 & 21 \\
\hline & 3 & 3 & & $0.125^{\prime \prime}$ & 7 & 7 & 7 & 21 \\
\hline & 4 & 3 & & $0.125^{\prime \prime}$ & 8 & 8 & 8 & 24 \\
\hline \multirow[t]{4}{*}{54} & 3 & 2 & & $0.125^{\prime \prime}$ & 7 & 8 & 8 & 23 \\
\hline & 3 & 3 & & $0.125^{\prime \prime}$ & 8 & 8 & 7 & 23 \\
\hline & 4 & 3 & & $0.125^{\prime \prime}$ & 8 & 8 & 8 & 24 \\
\hline & 5 & 3 & & $0.125^{\prime \prime}$ & 8 & 7 & 8 & 23 \\
\hline \multirow[t]{3}{*}{55} & 3 & 1 & & $0.125^{\prime \prime}$ & 8 & 7 & 7 & 22 \\
\hline & 3 & 2 & & $0.125^{\prime \prime}$ & 7 & 7 & 6 & 20 \\
\hline & 3 & 3 & & $0.125^{\prime \prime}$ & 8 & 8 & 7 & 23 \\
\hline 56 & Uncontrollable & & & & & & & \\
\hline
\end{tabular}

Assessing Table 2, it is evident that for the first few temperatures, scores assigned for initial adhesion and uniform distribution were extremely low. After many trials, it was 
determined that the wax was extremely sensitive to a minute increase in heat. The maximum temperature for the usefulness of the wax, with respect to this project, was found to be $55^{\circ} \mathrm{C}$. Figure 28 illustrates the maximum score per temperature versus the temperature for $1 / 8$ " diameter nozzle with a $1 / 8$ " gap and a metallic working surface. It can be seen that for the $1 / 8$ " nozzle diameter with a gap of $1 / 8$ " the best scores were produced around the $53^{\circ} \mathrm{C}$ to $54^{\circ} \mathrm{C}$ temperatures. At these temperatures, the wax is in a quasi-liquid solid state where it is controllable and where it will meet all the required criteria. Furthermore, since the nozzle was $1 / 8$ " in diameter, results with a gap of $1 / 8$ " between the extrusion head and the working table were noticed to be better. This piece of information was quickly documented.

\subsubsection{Phase 1 Conclusion}

Reviewing the results of the table once again, it was noticed that the most immediate problems were due to the initial adhesion of the wax to the working table and the problem of overflowing. It was hoped that solving the overflowing problem would somehow help to alleviate the initial adhesion concern. Since, the initial diameter of the nozzle used for the first half of the testing process was an eighth of an inch (1/8"), it was suggested that a smaller diameter nozzle might hinder the overflow of wax. Hindering the overflow of wax might in turn allow the wax to grip more securely to the working table as the extrusion head moved around.

\subsubsection{Testing Phase 2}

A nozzle with a diameter of one sixteenth of an inch (1/16") was machined and replaced with the original nozzle. The experiment was once again carried out. However, 
realizing the pattern from the initial testing phase, the experiments were concentrated around the $52{ }^{\circ} \mathrm{C}$ to $55^{\circ} \mathrm{C}$. Furthermore, since the nozzle diameter and the gap size for the previous experiment had a direct relationship, more emphasis was placed on the 0.0625 " gap while using the 0.0625 " diameter nozzle.

\subsubsection{Phase 2 Results}

Table 3 documents the results of the second phase of the variable optimization process. Although the focus of this phase wax concentrated around a gap size of 0.0625 ", some experiments were carried out with a gap size of 0.125 " in order to further enforce the hypothesis that gap size and nozzle diameter have a direct influence on each other in this experiment.

Table 3: Score Sheet Based on 1/16" Nozzle Diameter and Metallic Table Surface

\begin{tabular}{|c|c|c|c|c|c|c|c|c|}
\hline \multicolumn{9}{|c|}{$\begin{array}{l}\text { I.A. Initial Adhesion } \\
\text { U.D. Uniform Distribution } \\
\text { O.F. Over Flowing }\end{array}$} \\
\hline $\begin{array}{l}\text { Temp. } \\
\left({ }^{\circ} \mathrm{C}\right)\end{array}$ & $\begin{array}{l}\text { Feed-rate } \\
\text { ("//min) }\end{array}$ & Pump-rate & Gap & Gap & I.A. $(/ 10)$ & $\begin{array}{l}\text { U.D. } \\
\text { (110) }\end{array}$ & O.F. $(110)$ & Score \\
\hline \multirow{4}{*}{52} & 3 & 2 & $00625^{\prime \prime}$ & & 6 & 6 & 3 & 15 \\
\hline & 3 & 2 & & $0125^{\prime \prime}$ & 6 & 6 & 6 & 18 \\
\hline & 3 & 3 & & $0.125^{\prime \prime}$ & 6 & 6 & 7 & 19 \\
\hline & 4 & 3 & & $0.125^{\prime \prime}$ & 6 & 6 & 7 & 19 \\
\hline \multirow[t]{4}{*}{53} & 2 & 3 & $0.0625 "$ & & 7 & 7 & 8 & 22 \\
\hline & 3 & 2 & $0.0625^{\prime \prime}$ & & 7 & 7 & 8 & 22 \\
\hline & 3 & 3 & $0.0625 "$ & & 7 & 7 & 8 & 22 \\
\hline & 4 & 3 & $0.0625 "$ & & 8 & 8 & 9 & 25 \\
\hline \multirow[t]{4}{*}{54} & 3 & 2 & $0.0625^{\prime \prime}$ & & 7 & 8 & 9 & 24 \\
\hline & 3 & 3 & $0.0625^{\prime \prime}$ & & 8 & 8 & 8 & 24 \\
\hline & 4 & 3 & $0.0625 "$ & & 8 & 8 & 9 & 25 \\
\hline & 5 & 3 & $0.0625^{\prime \prime}$ & & 8 & 7 & 9 & 24 \\
\hline \multirow[t]{3}{*}{55} & 3 & 1 & $0.0625^{\prime \prime}$ & & 8 & 7 & 8 & 23 \\
\hline & 3 & 2 & $0.0625^{\prime \prime}$ & & 7 & 7 & 8 & 22 \\
\hline & 3 & 3 & $0.0625^{\prime \prime}$ & & 8 & 8 & 8 & 24 \\
\hline
\end{tabular}


Analyzing Table 3, it is quite clear that the reduction in the nozzle diameter greatly reduced the overflowing wax problem. Furthermore, Figure 29 illustrates the maximum score per temperature versus the temperature for a $1 / 16$ " nozzle diameter and a $1 / 16$ " gap for a metallic working surface. In this second round of experiments, the hypothesis that gap size and nozzle diameter have a direct impact on each other, was once again reaffirmed with the scores attributed to a gap size of 0.125 " with respect to those given to a gap size of $0.0625 "$

\section{Maximum Score Per Temperature Vs. Temperature (0.0625" Gap, 1/16" Nozzle Diameter)}

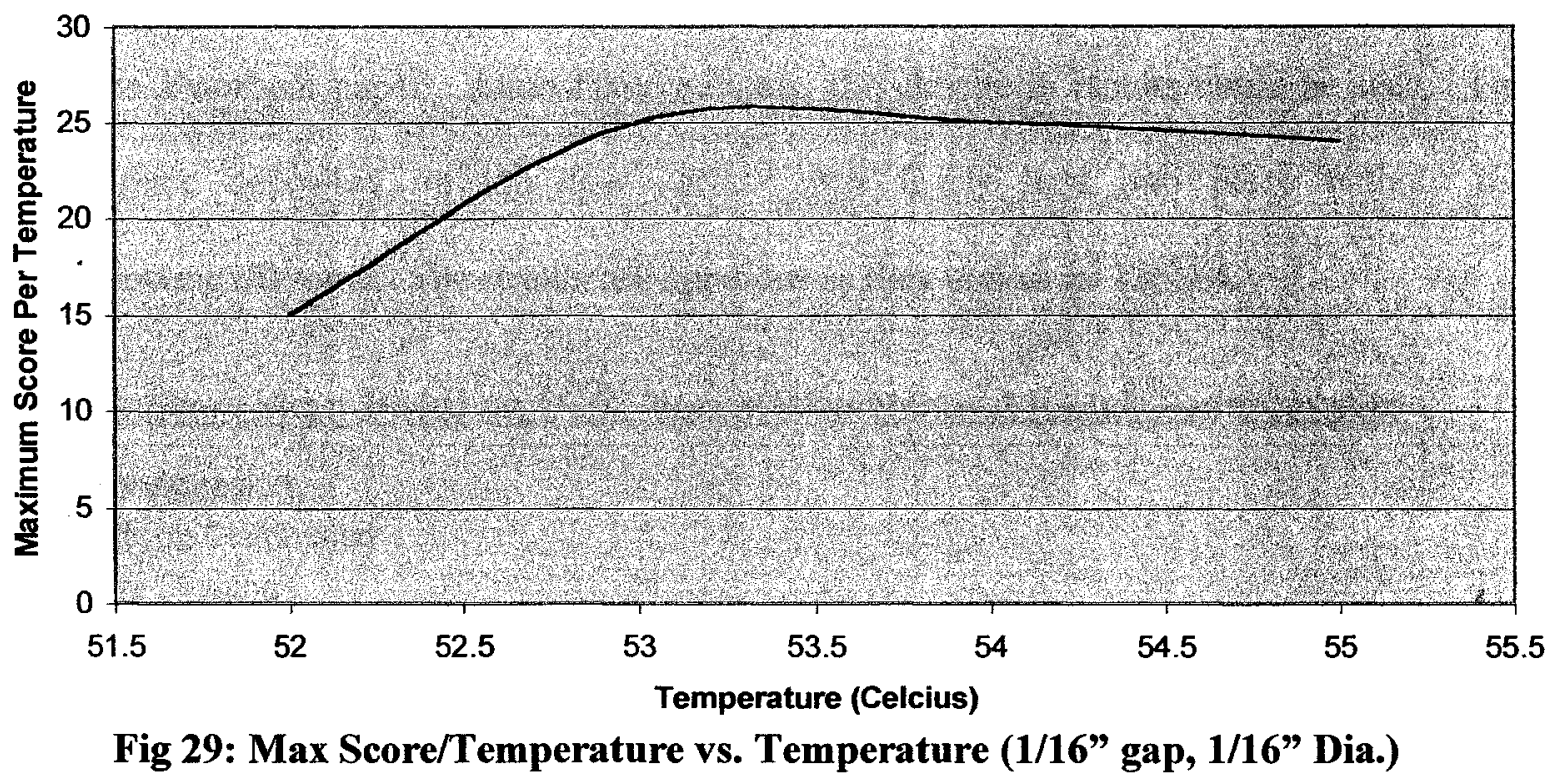

\subsubsection{Phase 2 Conclusion}

It is clear that higher scores were reached with a nozzle diameter of $1 / 16$ " and a gap of $1 / 16$ " between the extrusion head and the working table when compared to the scores achieved from the $1 / 8$ " nozzle diameter and gap. However, the enthusiasm to solve two problems with one method came to an end. The smaller nozzle diameter had no effect on 
the initial adhesion quality of the wax. The wax, once extruded, solidified without sticking to the working table and was thus dragged around as the extrusion head executed its tool path, hence the lower marks awarded for that criterion. The odd times when the wax seemed to have bonded to the working surface, quickly fell apart once the machining phase began.

\subsubsection{Phase 3}

It was hypothesized that a coarser textured surface with a higher coefficient of friction would help grip the wax once the extrusion head was in motion. Therefore, a very strong adhesive substance was sprayed on a piece of wood and was covered with a roughsurface absorbent paper. Almost immediately, the change in results could be seen. The wax once deposited on the new surface was firmly held into place without the overflowing dilemma. The results are apparent as they appear in Table 4.

Table 4: Score Sheet Based on 1/16" Nozale Diameter and Rough Paper Surface

\begin{tabular}{|c|c|c|c|c|c|c|c|c|}
\hline \multicolumn{9}{|c|}{$\begin{array}{l}\text { I.A. Initial Adhesion } \\
\text { U.D. Uniform Distribution } \\
\text { O.F. Over Flowing }\end{array}$} \\
\hline $\begin{array}{c}\text { Temp. } \\
\left({ }^{\circ} \mathrm{C}\right)\end{array}$ & $\begin{array}{c}\text { Feed-rate } \\
\text { ("'/min) }\end{array}$ & Pump-rate & Gap & Gap & I.A. (/10) & U.D. (110) & O.F. (/10) & Total \\
\hline \multirow[t]{6}{*}{53} & 4 & 3 & & $0.125^{\prime \prime}$ & 9 & 8 & 8 & 25 \\
\hline & 3 & 3 & & $0.125^{\prime \prime}$ & 9 & 7 & 6 & 22 \\
\hline & 4 & 2 & $0.0625^{\prime \prime}$ & & 8 & 6 & 6 & 20 \\
\hline & 3 & 2 & & $0.125^{\prime \prime}$ & 8 & 6 & 7 & 21 \\
\hline & 4 & 3 & $0.0625^{\prime \prime}$ & & 10 & 10 & 9 & 29 \\
\hline & 5 & 3 & $0.0625^{\prime \prime}$ & & 9 & 9 & 9 & 27 \\
\hline \multirow[t]{4}{*}{54} & 3 & 2 & $0.0625 "$ & & 9 & 8 & 8 & 25 \\
\hline & 3 & 3 & $0.0625^{\prime \prime}$ & & 9 & 8 & 7 & 24 \\
\hline & 4 & 3 & $0.0625^{\prime \prime}$ & & 10 & 10 & 9 & 29 \\
\hline & 5 & 3 & $0.0625^{\prime \prime}$ & & 9 & 7 & 8 & 24 \\
\hline \multirow[t]{3}{*}{55} & 3 & 1 & $0.0625^{\prime \prime}$ & & 9 & 7 & 7 & 23 \\
\hline & 3 & 2 & $0.0625^{\prime \prime}$ & & 9 & 7 & 6 & 22 \\
\hline & 3 & 3 & $0.0625^{\prime \prime}$ & & 9 & 8 & 7 & 24 \\
\hline
\end{tabular}


Maximum Score Per Temperature vs. Temperature (1/16" Nozzle Dia. 1/16" Gap, Rough Paper)

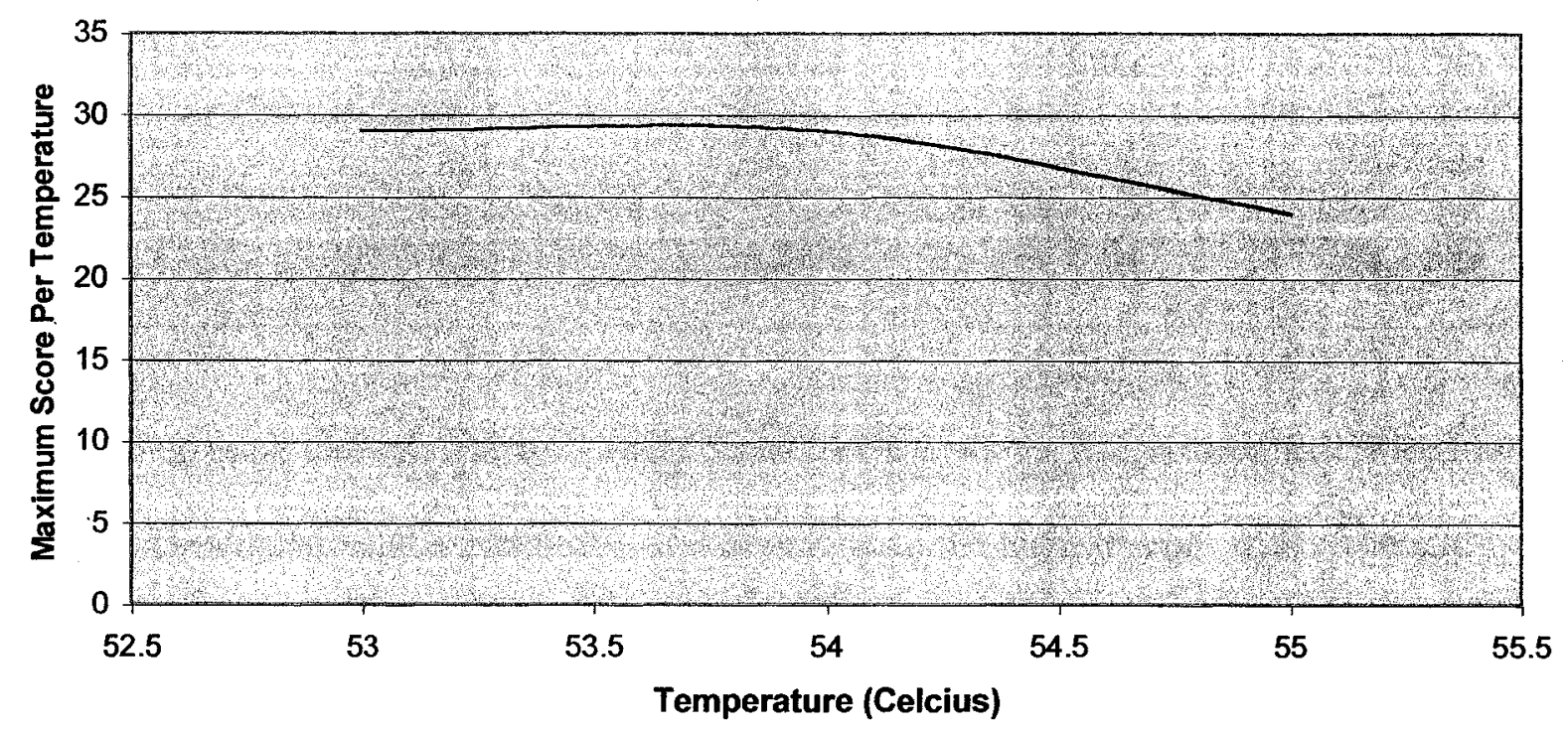

Fig 30: Max Score/Temperature vs. Temperature (1/16" gap, 1/16” Dia., Rough Surface)

It is visible from Figure 30, that the addition of the paper surface to increase friction between the wax and the working table was an instant success. The maximum possible score allowed for this decision matrix is 30 points. The rough paper raised the scores from previous trials to 29 points.

Furthermore, a maximum feed-rate and pump-rate were also assigned to allow both productivity and uniformity for the part being produced. The variable speed controller box has five speed settings as was shown in figure 23. However, only the latter three were used for testing due to their higher revolutions per minute. The lead screw connecting the plunger (piston) to the stepper is a $1 / 4-20$ UNC threaded rod. Table 5 indicates the RPM (revolutions per minute) of the stepper motor for each pump rate setting and also the travelling speed of the piston for each setting. 
Table 5: Stepper Motor Speeds and Feeds

\begin{tabular}{|c|c|c|c|c|}
\hline $\begin{array}{c}\text { Pump Rate } \\
\text { Speed } \\
\text { Setting }\end{array}$ & $\begin{array}{c}\text { RPM } \\
\text { (revolutions/min) }\end{array}$ & $\begin{array}{c}\text { Speed Of Piston } \\
\text { (Inches/mi) }\end{array}$ & $\begin{array}{c}\text { Nozzle Feed- } \\
\text { rate } \\
\text { in } \mathbf{3} / \mathbf{m i n}\end{array}$ & $\begin{array}{c}\text { Time to } \\
\text { empty full } \\
\text { load (min) }\end{array}$ \\
\hline 1 & 3.5 & 0.175 & 0.005 & $21.4 \mathrm{~min}$ \\
\hline 2 & 4.5 & 0.225 & 0.011 & $16.6 \mathrm{~min}$ \\
\hline 3 & 7.25 & 0.3625 & 0.047 & $10.3 \mathrm{~min}$ \\
\hline
\end{tabular}

Going back through Table 2, Table 3 and Table 4 above, it is noticed that a feed-rate of 4 inches/minute and a pump-rate of 3 always gave the highest score with respect to the other feed-rate and pump-rate combinations. Therefore, these two settings were used throughout the prototyping production stage. The last column in Table 5 illustrates the time it takes to empty a full cylinder. The cylinder can hold $0.414 \mathrm{in}^{3}$ of wax when filled right to the top and it takes 21.4 minutes to empty the cylinder on pump setting $1,16.6$ minutes to empty on pump setting 2 and 10.3 minutes to empty on pump setting 3 . It should also be noted that one full load in the cylinder can generate approximately 3 layers for a $1 " \times 1 " \times 0.250 "$ part. The next phase of the experiment dealt with the actual production of simple prototype parts.

\subsection{Fine Tuning Test Procedure}

\subsubsection{Temperature Adiustment}

Since these initial trial periods were performed on single lines and single layers, it was discovered later that the wax needed more time to reach a homogeneous of $54^{\circ} \mathrm{C}$. This was noticed once the testing was upgraded from a single layer, to a multi-layered cube and cylinder where vast amounts of wax were needed. Halfway through the programs, 
the wax would leak out uncontrollably much like it had done at $55^{\circ} \mathrm{C}$. The problem was that the step-less heat controller and the heating coils needed a sufficient amount of time to reach their prescribed temperature. Previously, when the program was executing its commands, it was noticed that the temperature would keep rising and as it rose, the melting temperature of the wax was reached and it poured out of the nozzle uncontrollably. Therefore, the temperature was lowered by $1{ }^{\circ} \mathrm{C}$ to $53^{\circ} \mathrm{C}$. Again, at this temperature the wax performed exceptionally well with respect to the required criteria and the previous trials documented above. At this temperature, the wax was also stable when testing was upgraded to that of an actual part.

\subsubsection{Prototype Production Problems and Solutions}

The production of simple geometric shapes, revealed new complications to over come. Firstly, although the coarser surface held the wax into place, it was however, not strong enough to secure the wax once the machining process had begun. The surface needed to have better adhesion in order to securely hold the wax into place while the end-mill machined the perimeter and surface of the shape. Therefore, the same adhesion spray that was used to bond the rough surface paper to the wood was also sprayed on top of the paper for better adhesion between the wax and the paper. This quick fix worked fairly well and it was apparent when the machining phase began that the deposited wax stayed fastened to the paper.

The second obstacle was evident when the second layer of wax was being deposited on the first layer. Since one layer had solidified and the other layer was in a solid-liquid state, the two layers could not fully bond together to create a homogenous continuity. 
Due to this problem, machining was obviously not properly performed and multiple test pieces were destroyed before a contingency idea was developed. It was suggested that if the first layer were not fully cured when the second layer was deposited, then the two would bond much better. Using the hot air gun on the first layer to keep the temperature high enough to allow the cohesion between the two layers first tested this idea. However, the sheer force and heat of the air literally melted and blew the layer away. The distance between the hot air gun and the first layer of wax was adjusted repeatedly to find a suitable range for optimum performance. Unfortunately, this method was not reliable for the reasons mentioned above and for the fact that once an optimum range was discovered, only one side of the layer was affected. Therefore a new idea had to be developed to allow uniform heat to be distributed throughout the layer without affecting its present position. It was hypothesized that a lit candle underneath the table would allow the heat to uniformly spread across the working table and onto the layer. The candle would not be strong enough to generate heat through the quarter inch thick aluminium table and through the quarter inch thick wood. Therefore, the wood was replace with a $16 \mathrm{GA}$ $(0.0625 ")$ piece of aluminium sheet metal and was clamped past the table to allow the candle to be place underneath the sheet metal only. After multiple iterations through trial and error, the most optimum distance between the candle and the sheet metal was found and implemented.

Finally, the Hybrid Rapid Prototyping system was calibrated, fully assembled and was prepared to carry out simple geometric shaping of wax prototypes. The next section of this report deals with the software portion of the experiment and some of the programs that were written to accommodate the production of wax prototypes. 


\subsection{Designs and Implementation of Software}

Visual Basic was used to write several programs for a variety of simple geometric shapes. These shapes include cubes and rectangular prisms, hollow cubes, solid cylinders

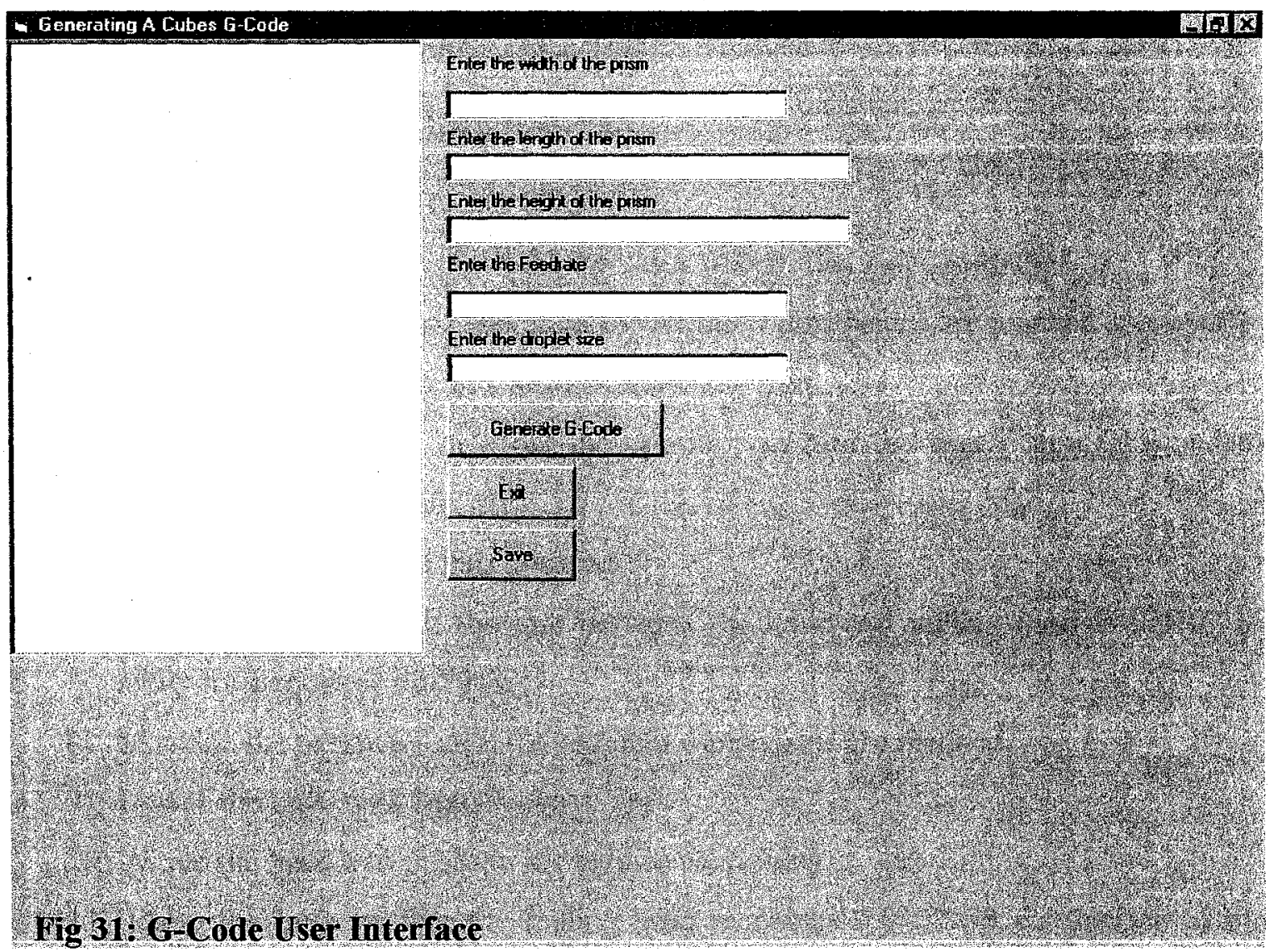

and a pyramidal shape. All the programs are listed in Appendix 3. The programs would essentially ask the user to input the desired dimensions such as length, width, and height. The start-up screen for the program is illustrated in Figure 31. The program would also require the user to input a feed-rate to determine the speed at which the wax extrusion head should move and finally a nozzle diameter size. This last criterion was supplemented in order to allow the operator a little range of versatility. If the diameter of the wax nozzle ever changed back to $1 / 8$ " then the program would compensate for that larger bead and would continue with the required output and vice versa for a smaller diameter. The program would operate in systematic prescribed steps. The following is a 
short description of the program. All the programs are based on the same format and so only one of them will be outlined. The following is a summary of executed commands from the cube prototype program once the initial starting point has been determined.

\section{PROGRAM STEPS FOR CUBE SHAPE}

1) Lower the table to the required height.

2) Dwell for 3 seconds to allow the operator to turn the wax piston $\mathrm{ON}$.

3) Extrude the first layer of wax until the required dimensions are reached.

4) Lower the table so that wax head is above the part.

5) Dwell for 30 seconds to allow the operator to turn the wax piston OFF and to allow the wax to solidify.

6) Travel the milling head into position.

7) Raise the table so that the gap between the table and milling head is 0.010 " to allow for perimeter machining.

8) Machine the perimeter until the required dimensions are reached.

9) Lower the table untll head is above part.

10) Move the head into position for surface machining.

11) Raise the table so that the milling head machines 0.020 " off the surface of the layer.

12) Machine the surface of the layer until the required dimensions are reached.

13) Lower the table until the milling head is above the part.

14) Travel the wax head back into home position.

15) Repeat steps until the height indicated by the user is reached.

For the hollow cube, the user would be prompted to input all the information stated above as well as the inner length and width dimensions. However, this program is limited to cubic shapes only. Meaning, the dimensions of the exterior square must be equal and the dimensions of the interior square must also be equal. Therefore, essentially 
the system is producing a square within a square if it was to be viewed on a twodimensional scale. For the solid rectangular prism program, the path of the tool is such that it moves from left to right, jumps a specified unit in the $y$-direction and then moves from right to left and jumps again in the y-direction.

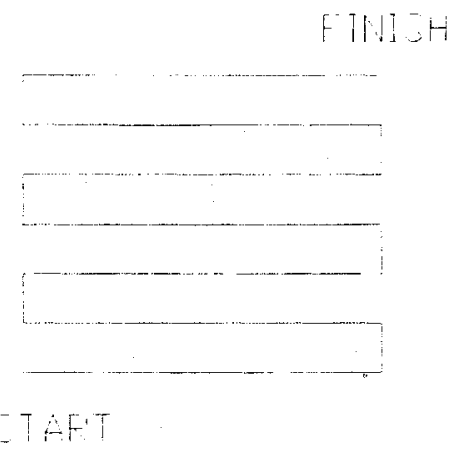

Fig 32: Tool Path For A Solid Rectangular Shape

This path was used as the building blocks for the program and is illustrated in figure 32 . Essentially a loop was created to repeat this path until the desired dimensions were reached.

The program to produce the cylindrical shapes is somewhat different with respect to the wax deposition head moving in a circular path from the outside in. For the solid cylinder the user is required to input a radius, a nozzle diameter, a feed-rate and the height of the cylinder. After the program has completed its calculations it will begin to extrude the wax in a circular fashion beginning with the outer dimension. The next path will be an inner circular path slightly smaller than the original and so on until the first layer is completed. The program will then begin to machine around the perimeter of the first layer and the top surface so that the next layer can be deposited on a flat surface. 
Thus reducing stair step inaccuracies. This process is continued until the final dimensions are reached.

Once the information is inputted into the program, the computer would create and return an output in standard G-code notation. The programs were also outfitted with a "Save" command that allows the user to save the G-code data as a text (.txt) file. This sub-program was very essential due to the fact that Visual basic 6 runs on the windows platform and DeskNC runs on the DOS platform. Therefore, two computers were used and the data had to be saved as a text file in Windows so that it can be transferred to the DOS program that runs the HRP system. Once the file was downloaded and all the system checks were accounted for, the machine began to produce the required part.

The next section of this project illustrates the HRP set-up procedure and the actual production of a prototype part. The steps for the HRP process were documented as pictures to better illustrate the actions that the system undertakes. 


\subsection{Visual Illustration of Production Steps}

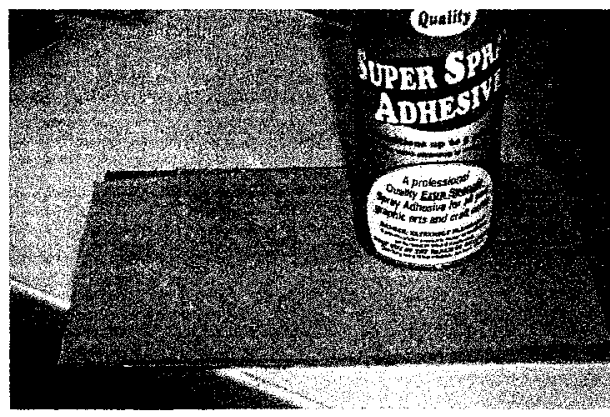

Fig 33: Step 1: Applying Adhesive

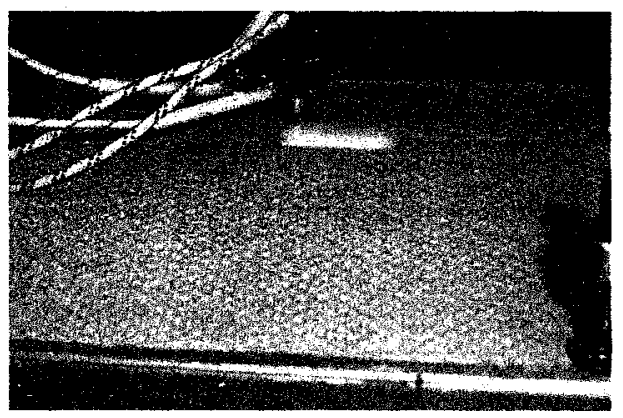

Fig 35: Step 3: Deposition Phase

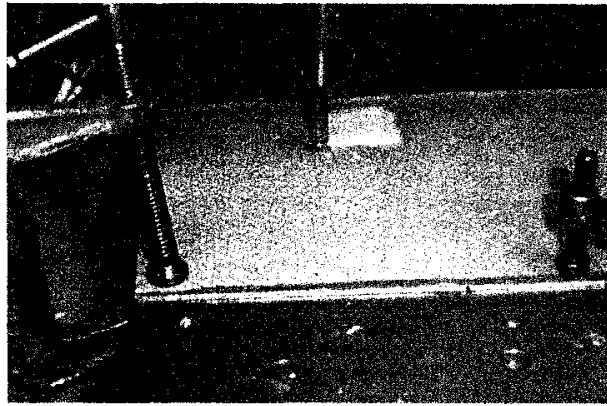

Fig 37: Step 5: End-mill position

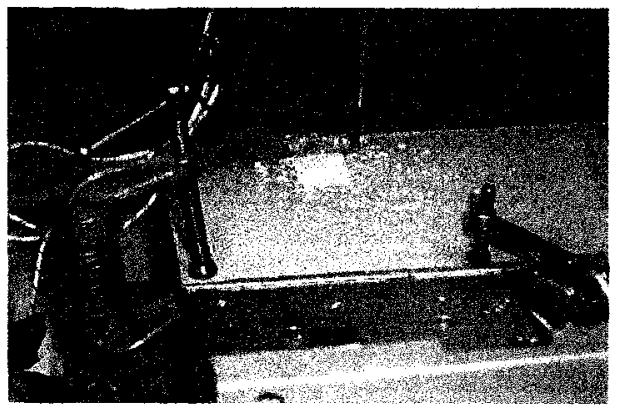

Fig 39: Step 7: Machining Surface Phase

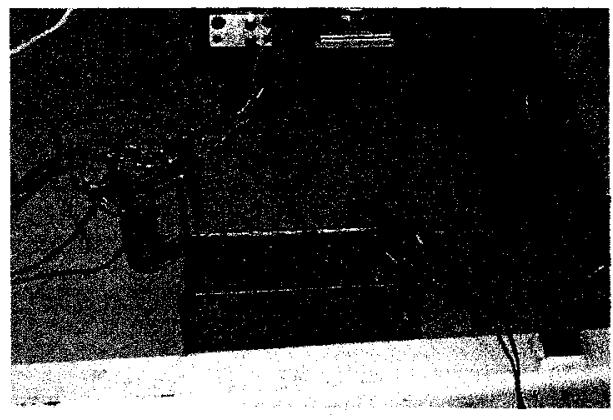

Fig 34: Step 2: Clamping Surface

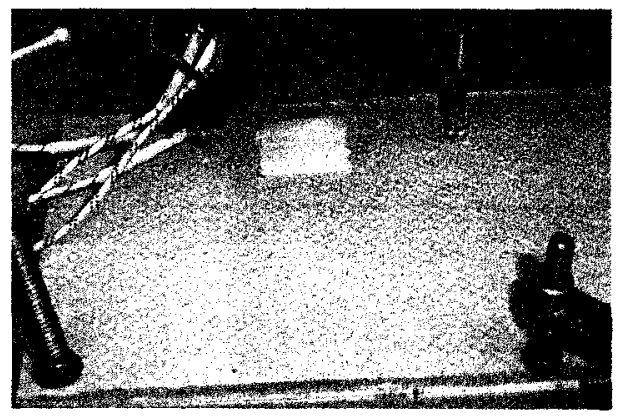

Fig 36: Step 4: Curing the Wax

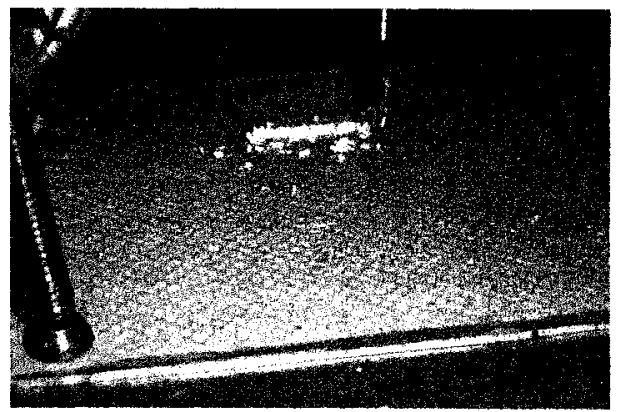

Fig 38: Step 6: Machining Perimeter Phase

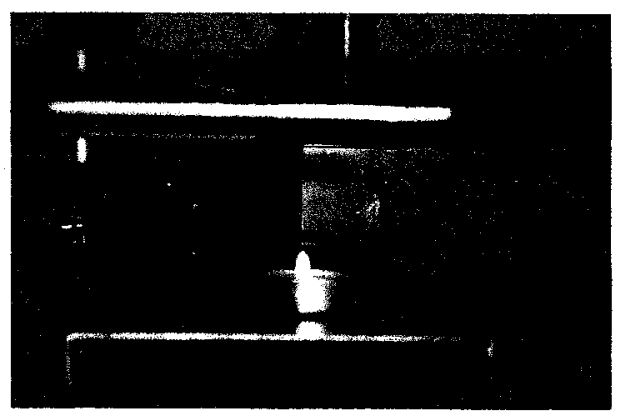

Fig 40: Step 8: Preparing Layer Bondage

Steps 3 thru 8 were repeated until desired dimensions were reached 


\section{CHAPTER 3 - RESULTS AND DISCUSSION}

\subsection{Cubic and Rectangular Prism Shapes}

Once the system was finely tuned and the software was debugged, the final phase of the research could commence. The production of simple wax prototypes including shapes such as cubes, cylinders, hollow cubes and pyramids could begin.

Table 6: Prototype Results For Cubical Shapes

\begin{tabular}{|c|c|c|c|c|c|c|c|c|c|c|c|c|}
\hline & \multirow{2}{*}{\multicolumn{3}{|c|}{$\frac{\text { Required }}{\text { Size (⿶) }}$}} & \multirow{2}{*}{\multicolumn{3}{|c|}{$\begin{array}{l}\text { Exact } \\
\text { Size (w) }\end{array}$}} & \multirow{2}{*}{\multicolumn{3}{|c|}{$\frac{\text { Tolerance }}{\text { (Inches) }}$}} & \multirow{3}{*}{\begin{tabular}{|c|} 
Edge \\
Ouality \\
(110) \\
\end{tabular}} & \multirow{3}{*}{\begin{tabular}{|c|} 
Surface \\
Quality \\
$(/ 10)$
\end{tabular}} \\
\hline & & & & & & & & & & & & \\
\hline Shape & Run & L & $\mathbf{w}$ & H & $\underline{L}$ & w & H & L & $\mathbf{w}$ & $\mathbf{H}$ & & \\
\hline \multirow{8}{*}{ Square } & & & & & & & & & & & & \\
\hline & 1 & 1.000 & 1.000 & & 1.067 & 1.076 & & 0.067 & 0.076 & & 7 & 8 \\
\hline & 2 & 1.000 & 1.000 & & 1.010 & 0.986 & & 0.010 & 0.014 & & 6 & 8 \\
\hline & 3 & 0.500 & 1.000 & & 0.490 & 1.035 & & 0.010 & 0.035 & & 8 & 8.5 \\
\hline & 4 & 0.750 & 0.750 & & 0.756 & 0.745 & & 0.006 & 0.005 & & 8 & 9 \\
\hline & 5 & 1.000 & 1.000 & & 1.025 & 1.035 & & 0.025 & 0.035 & & 8 & 8.5 \\
\hline & & & & & & & AVG: & 0.024 & 0.033 & & 7.4 & 8.4 \\
\hline & & & & & & & & & & & & \\
\hline \multirow[t]{5}{*}{ Cube } & 1 & 1.000 & 1.000 & 0.250 & 1.035 & 1.020 & 0.236 & 0.035 & 0.020 & 0.014 & 6 & 8 \\
\hline & 2 & 1.000 & 1.000 & 0.250 & 1.027 & 1.012 & 0.241 & 0.027 & 0.012 & 0.009 & 6.5 & 7 \\
\hline & 3 & 0.500 & 1.000 & 0.250 & 0.487 & 1.037 & 0.230 & 0.013 & 0.037 & 0.020 & 8 & 8.5 \\
\hline & 4 & 0.750 & 0.750 & 0.250 & 0.770 & 0.752 & 0.241 & 0.020 & 0.002 & 0.009 & 7.5 & 8 \\
\hline & & & & & & & AVG: & 0.024 & 0.018 & 0.013 & 7.0 & 7.9 \\
\hline
\end{tabular}

Table 6 depicts the results acquired from the production of a one-layered square shaped object and a multi-layered cubical object. The results in Table 6 are of those objects that were not damaged during the removal and measurement recording stage. The first three columns after the trial column represents the intended measurements required. The next three columns illustrate the measurements taken from a digital calliper after production had been completed and the last two columns were assigned to give a subjective view on the edge quality and surface quality of the parts being produced. It is clear after reviewing the table, that the actual shapes produced were not perfectly square. On average, the two sides of the square were off by roughly 0.010 " and the surface quality was noted to be better than the edge quality. To account for the difference in 
quality between the edge and the surface, it should be noted that once experimentation had begun, the end-milling head was not spinning in a concentric circle. There was a tiny bit of wobble that was noted and during that stage was judged to be negligible in consequence. However, the difference in quality and for that matter dimensional size cannot fully be responsible for the characteristics of the end-milling station. Throughout the experimentation the stepper motors used for the $\mathrm{X}$ and $\mathrm{Y}$-axes would periodically step or pulse without the required commands. This was attributed to the heavy load of electrical power that was being supplied from one terminal. Using a six-station power bar, electrical power from one outlet was diverted to the main computer, the monitor, the I/O board, hot-air gun, stepper motor for the piston and the electrical power supply for end milling. The electrical load placed on this terminal would sometimes deactivate the electrical power supply for the end milling head and would occasionally force the stepper motors to pulsate in a certain direction while the program was being executed. Thus, the inaccuracies and edge quality obtained can be credited to the lack of proper power distribution. This issue was addressed however, by halting the melting of wax during production runs and by turning the end milling power supply off when it was not in use.

Figures 41 and 42 illustrate the evolution of the HRP system. In Figure 41, the first piece produced is displayed on the far left hand side. The sides are clearly not square and the surface is shown to be bumpy and uneven. After several iterations the next piece (second from the left) was produced. This piece was machined more thoroughly than the previous piece, yet the edges still did not have the satisfactory quality and appearance that was required during the experimental proposal. 


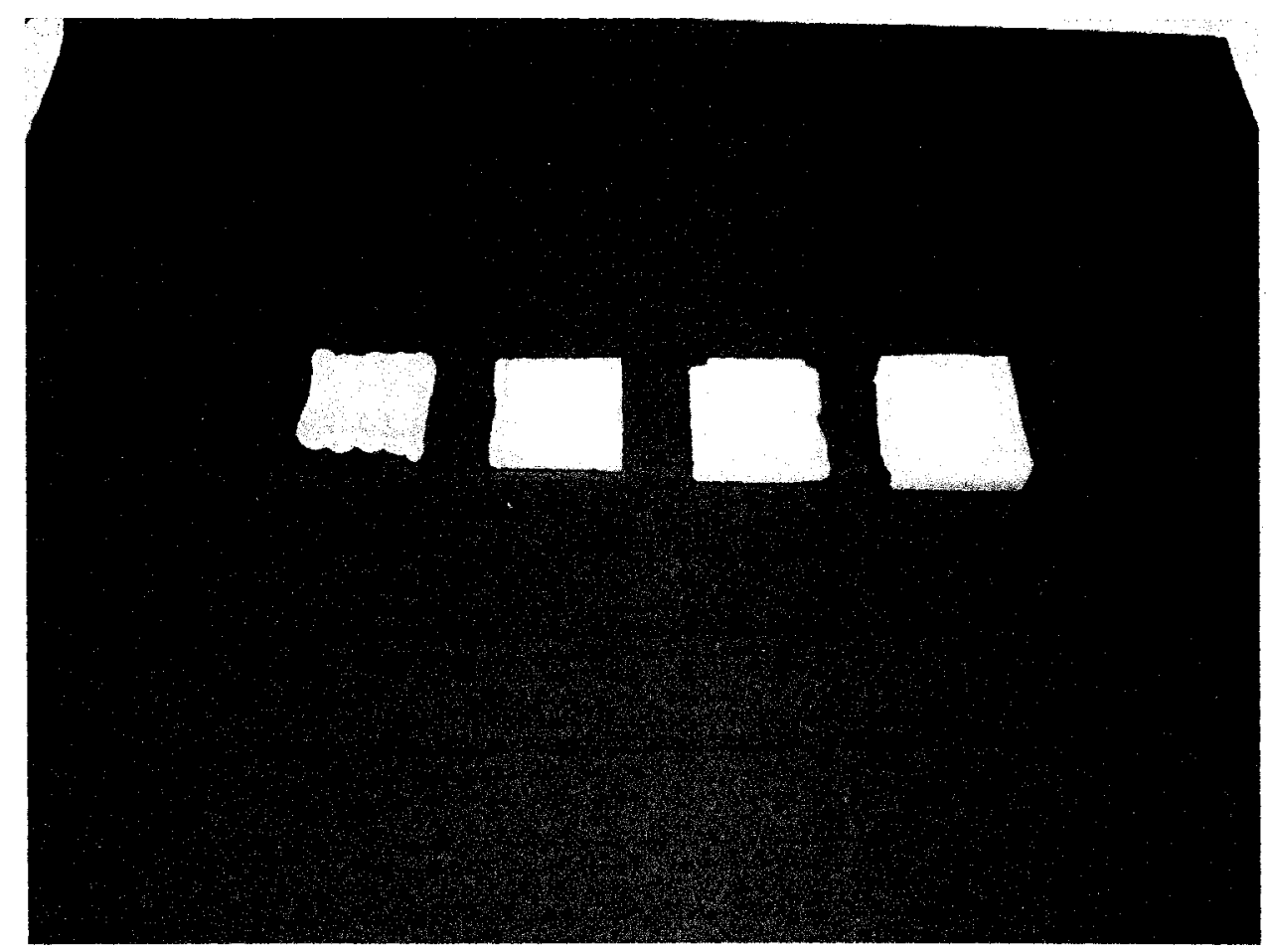

Fig 41: View of Square Prototypes

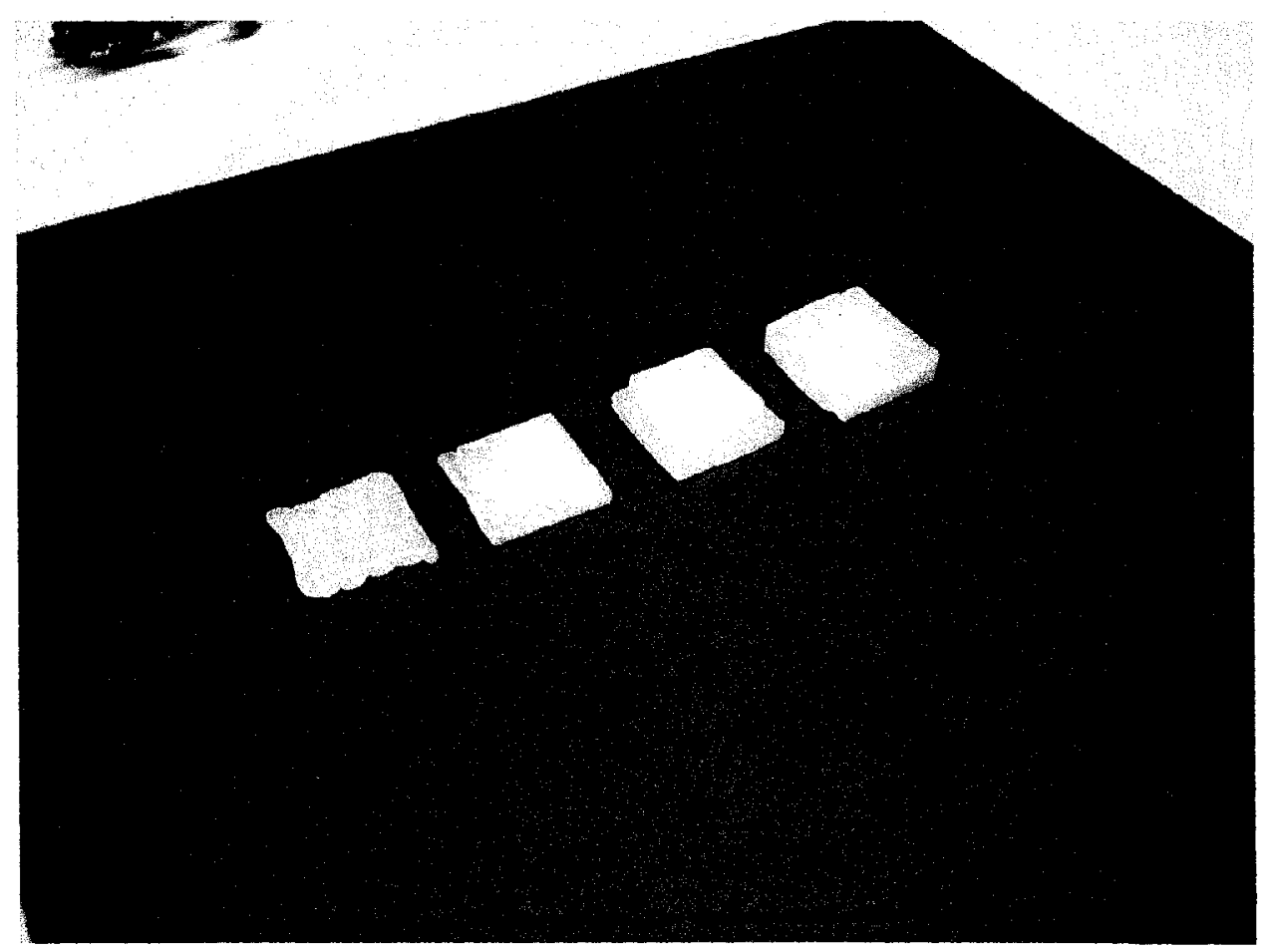

Fig 42: Angled View of Square Prototypes

The third (third from right) visual model represented the successful completion of multiple layers of wax deposited and machined to a near perfect square with edge quality 
and top surface quality which was deemed to be acceptable. A 4-layered cubical shape followed this progression, while maintaining the same dimensional accuracy, edge quality and top surface smoothness that was witnessed in its three-layered pyramidal predecessor. Following the completion of the cubical shape, the software was instructed to produce a series of rectangular prisms also. Again, the evolution of the shapes can be seen in Figure 43. The piece on the far left shown in Fig 43 is not fully rectangular and the surface quality is clearly not acceptable. However, after multiple iterations and the completion of the fine-tuning process the pieces thereafter were deemed more acceptable with respect to accuracy and surface finish.

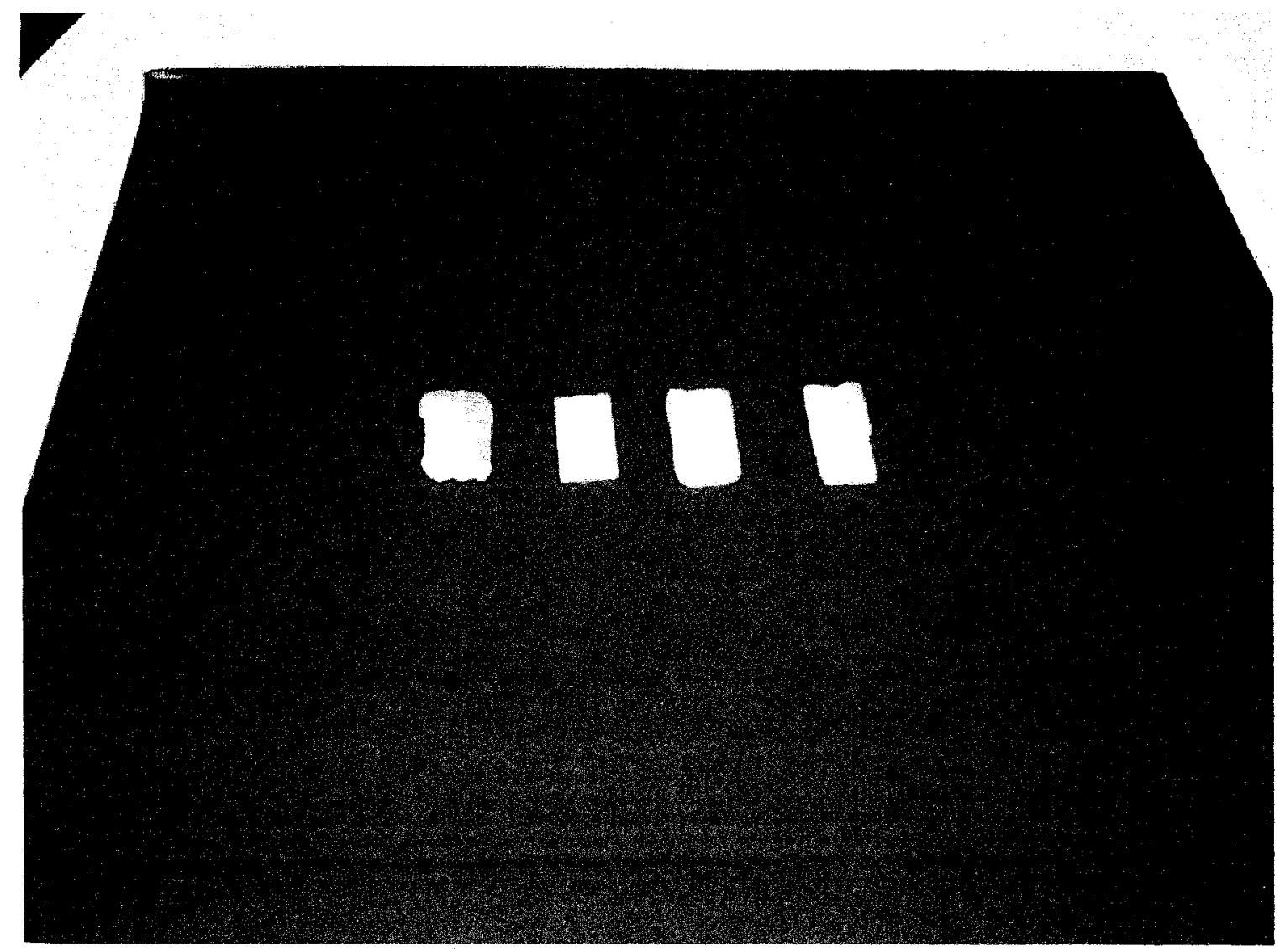

Fig 43: View of Rectangular Wax Prototypes 


\subsection{Circular and Cvlindrical Shapes}

The next shape in the experimentation phase was a one-layered circle and a multilayered cylinder. Referring to Table 7, the overall accuracies remained constant with respect to the cubical shapes except that the edge quality and surface scores showed higher gains over the duration of this experiment than the previous trials. This can be directly linked to the fact that the feed-rate and the stepper motor pump rate were reduced during this section.

Table 7: Prototype Results For Cylindrical Shapes

\begin{tabular}{|c|c|c|c|c|c|c|c|}
\hline & & Required & Exact & & Iolerance & Edge & Surface \\
\hline & & Diameter & Diameter & & (Inches) & Quality & Quality \\
\hline Shape & Irials & (Inches) & Inches) & & & (I10) & (I10) \\
\hline \multirow[t]{6}{*}{ Circle } & 1 & 1.000 & 1.038 & & 0.038 & 5 & 8 \\
\hline & 2 & 1.000 & 1.026 & & 0.026 & 5 & 7 \\
\hline & 3 & 0.750 & 0.732 & & 0.018 & 9 & 9 \\
\hline & 4 & 0.750 & 0.735 & & 0.015 & 8.5 & 9 \\
\hline & 5 & 1.000 & 1.011 & & 0.011 & 8.5 & 9 \\
\hline & & & & AVG: & 0.018 & 7.8 & 8.5 \\
\hline & & & & & & & \\
\hline \multirow[t]{5}{*}{ Cylinder } & 1 & 1.000 & 1.026 & & 0.026 & 6 & 8 \\
\hline & 2 & 1.000 & 1.037 & & 0.037 & 7 & 8 \\
\hline & 3 & 0.750 & 0.736 & & 0.014 & 8 & 8 \\
\hline & 4 & 0.750 & 0.741 & & 0.009 & 8.5 & 8 \\
\hline & & & & AVG: & 0.022 & 7.4 & 8.0 \\
\hline
\end{tabular}

In the early stages of production it was observed that there was a major difference between the production of cubical shapes and that of cylindrical shapes. For the production of cubical shapes, only one axis was being used during a direction change (for example from the $\mathrm{X}$ axis to the $\mathrm{Y}$ axis). For the production of cylindrical shapes, both axes had to be in motion in order to execute a curved line. The vibrations generated by the simultaneous movement of both axes gave slightly worst results that those generated by the production of squares, which only use one axis at a time. Using a feed rate of 4 inches/minute in combination with the movements of the $\mathrm{X}$ and $\mathrm{Y}$-axes, it was evident 
that there was too much force being generated on the system for any meaningful results. Thus the feed rate was reduced to 3 inches/minute in order to reduce the vibrations, which caused miss-steps or pulses in the system. Since it was discovered that the feed rate and the stepper motor pump rate had a direct relationship, the stepper motor's speed was reduced from a speed of 3 (7.25 revolutions/minute) to a speed of 2 (4.5 revolutions/minute). The improvements in dimensional accuracy as well as edge and surface quality are clearly visible in Table 7. The reduction in feed-rate, helped to ease the forces caused by the system's simultaneous motions in the $\mathrm{X}$ and $\mathrm{Y}$-axes during the cylindrical production runs so that pulses or steps being generated by the stepper motors would only be executed at the operators request and not by the forces of vibration.

Figures 44 and 45 depict the advancement of the cylindrical wax prototype production series relating to accuracy and surface finish. Figure 44 shows one-layered prototypes that were produced without machining. The first three prototypes are that of a 1-inch diameter, 1-layered circle. The first piece (far left) had rough edges and an unfinished top surface. The next three prototypes highlight the final approved samples that had an acceptable edge finish, top surface finish, and had dimensionally acceptable accuracies. Referring to figure 45 , the two prototypes on the left were multi-layered cylinders $(3 / 4$ " diameter). Again, it can be noticed that the middle prototype was slightly more round on the edges than its predecessor on its left hand side. However, both these prototypes displayed great surface quality in terms of smoothness. Finally, the last prototype illustrated in figure 45 , shows the extruded outline for a circular shape two inches in diameter. If the program had continued, then subsequently smaller circles would have been extruded until the first layered was complete. 


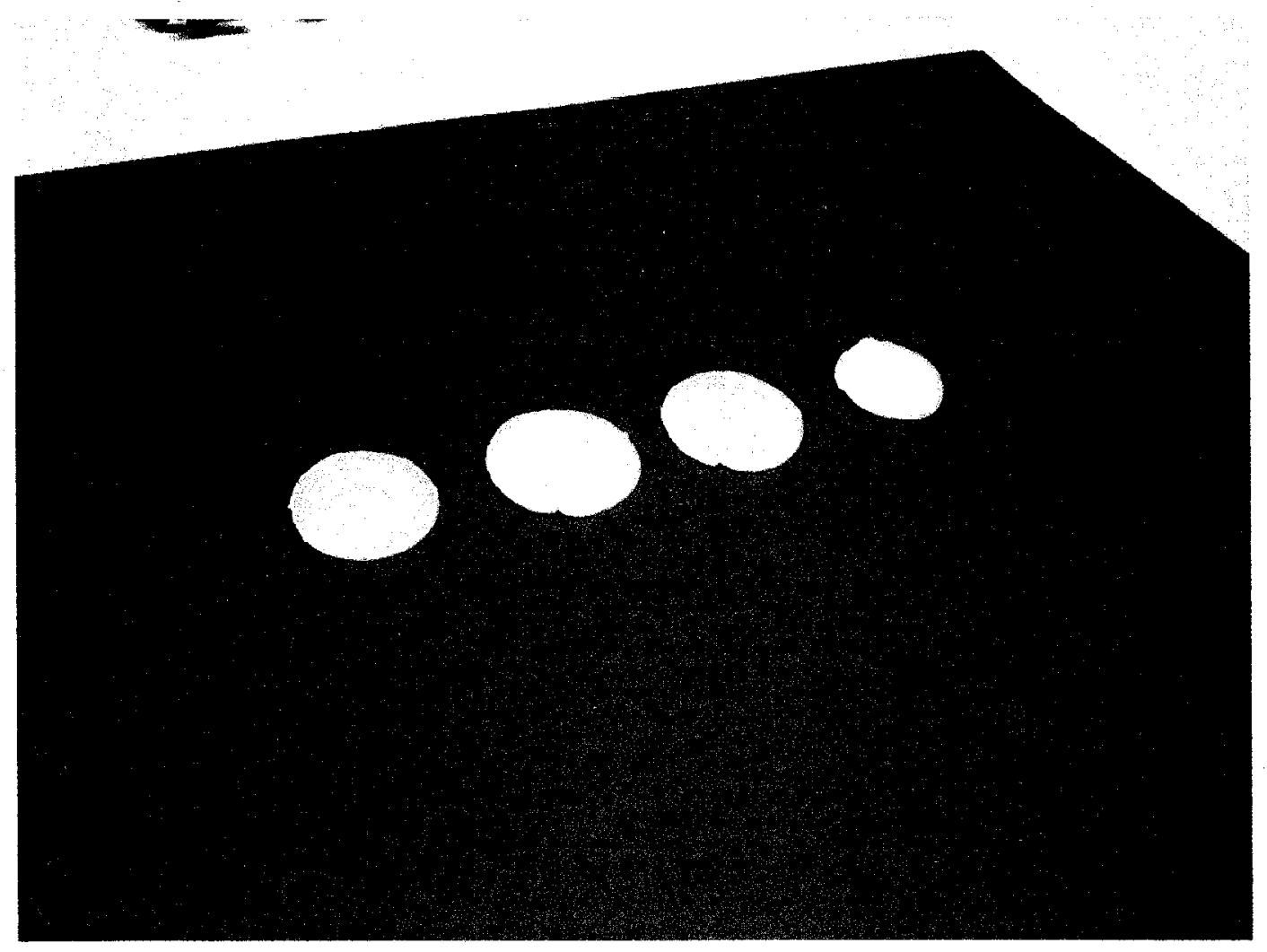

Fig 44: One-layered Circular Prototypes

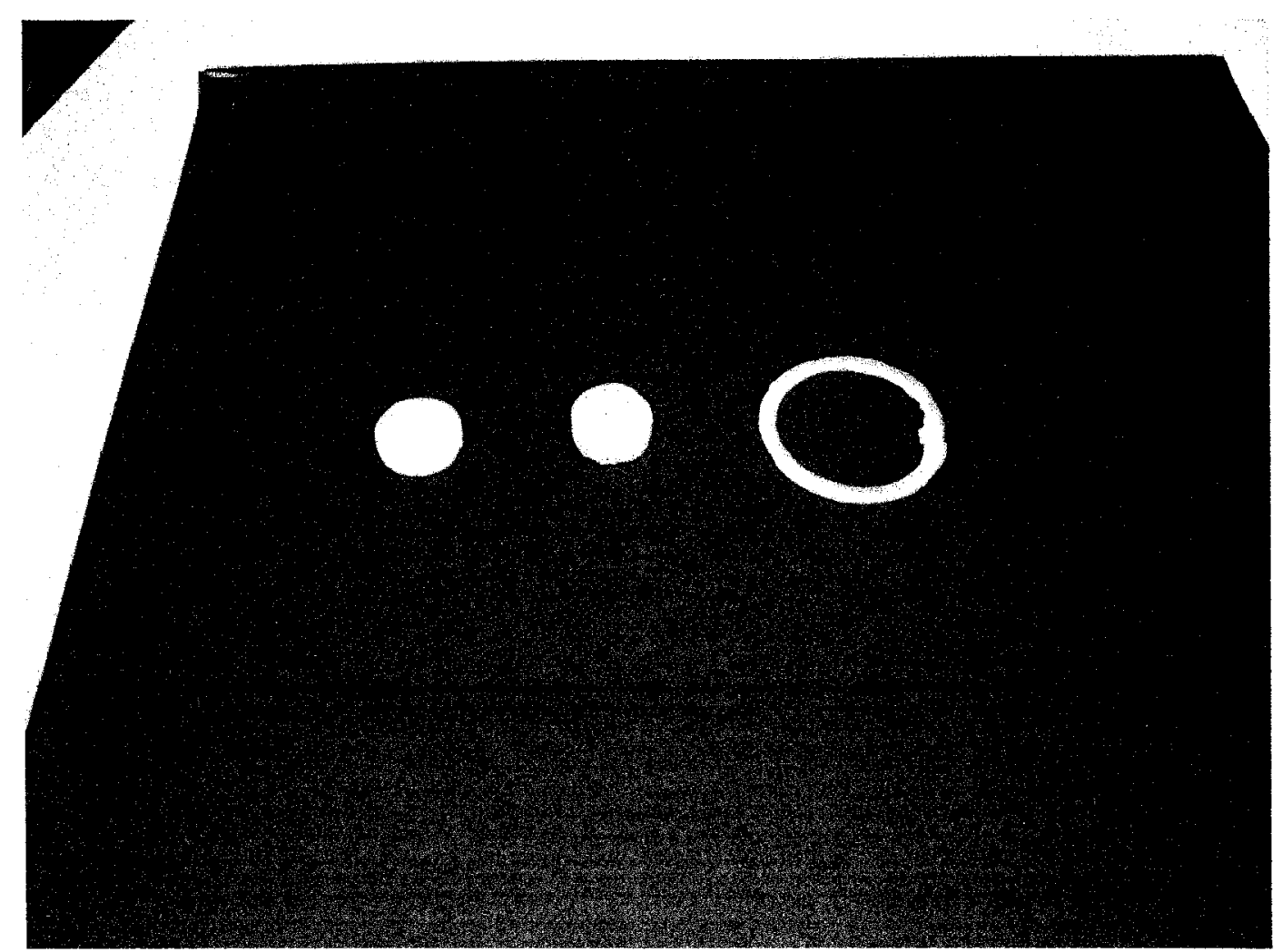

Fig 45: Multi-layered Circular Prototypes 


\subsection{Hollow Cubes and Pyramidal Shapes}

The last two shapes produced during this phase of the experiment were the pyramidal shape and the hollow cube shape (Figures 46-48). These two shapes were introduced to demonstrate the capabilities of the HRP system. As a result, no data was compiled for these two shapes with respect to precision and tolerance. Figure 46 shows multiple

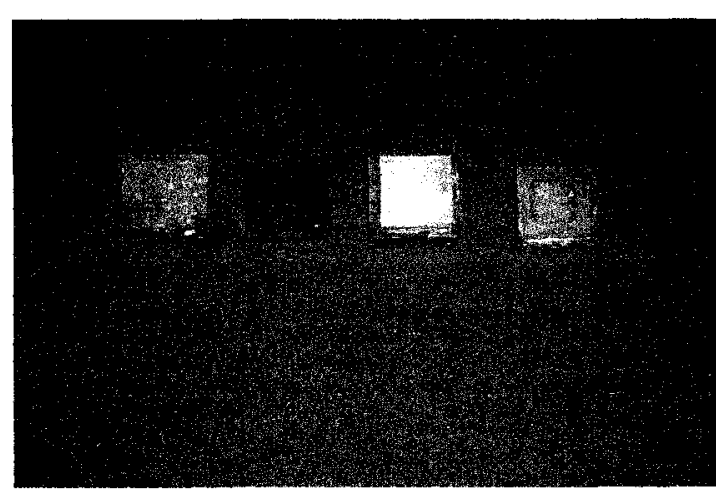

Fig 46: Top View Pyramidal Shape

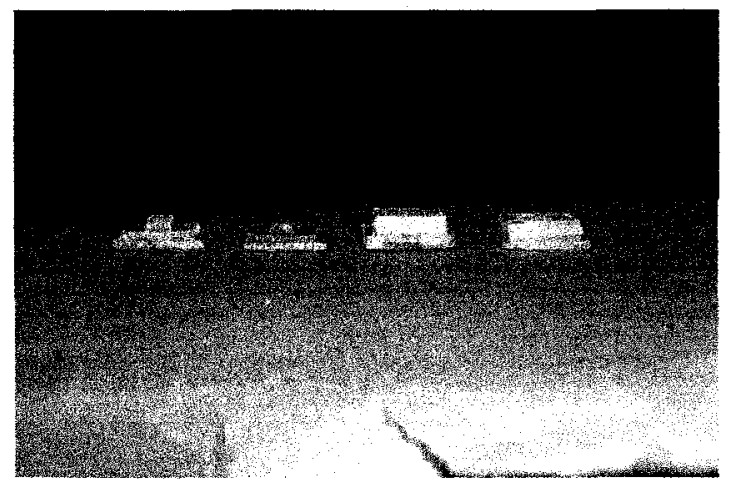

Fig 47: Side View Pyramidal Shape

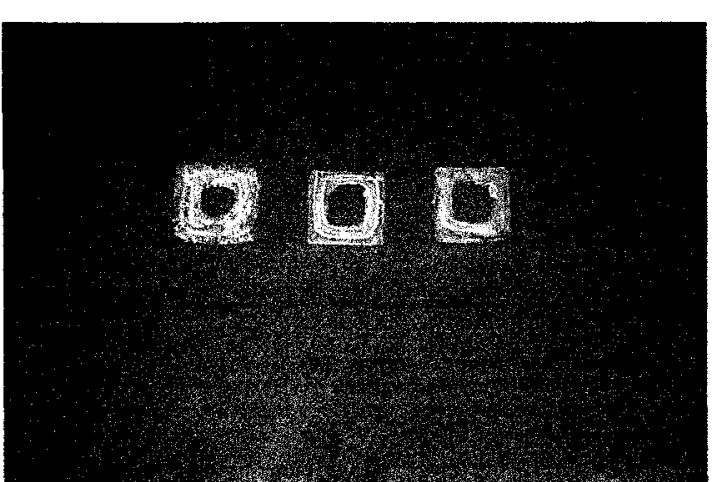

Fig 48: Top View Hollow Square samples of different pyramidal shapes and it also illustrates a hollow pyramidal shape.

The first two shapes (from left to right) resemble two-step, three-platform pyramids. Figure 47 depicts the side views of the pyramidal shapes. From a human eye perspective with respect to Figure 49 , it can be seen that the base of the pyramid, the middle of the pyramid and the top of the pyramid all lie square to each other. The third shape is a one-step, two-platform pyramidal shape. Again, these shapes were produced not only to demonstrate the ability of the system, but also that of the developed software. The hollow cubes (Figure 48) produced had the same outer dimensional accuracies and tolerances as the solid cubes mention earlier. However, the inner portion 
of the cube did not entirely resemble a mathematical square. The corners had a slight radius as would be expected when using a router bit or an end-milling bit.

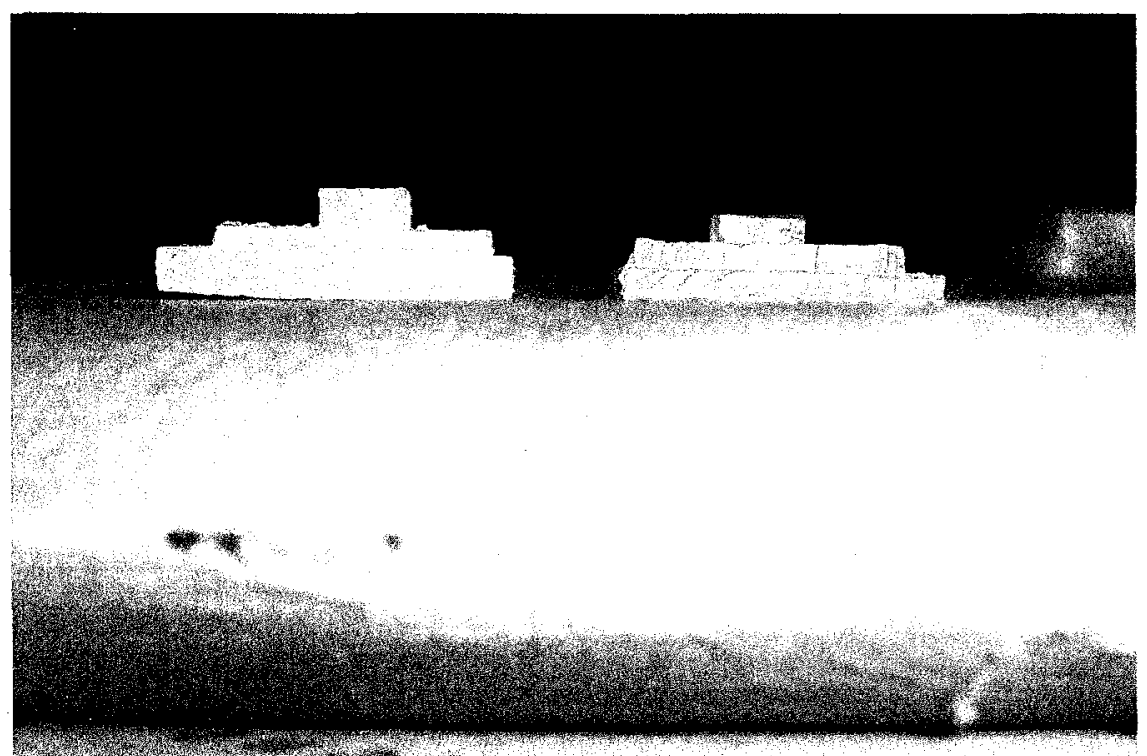

Fig 49: Zoom-in view of Pyramid Shape

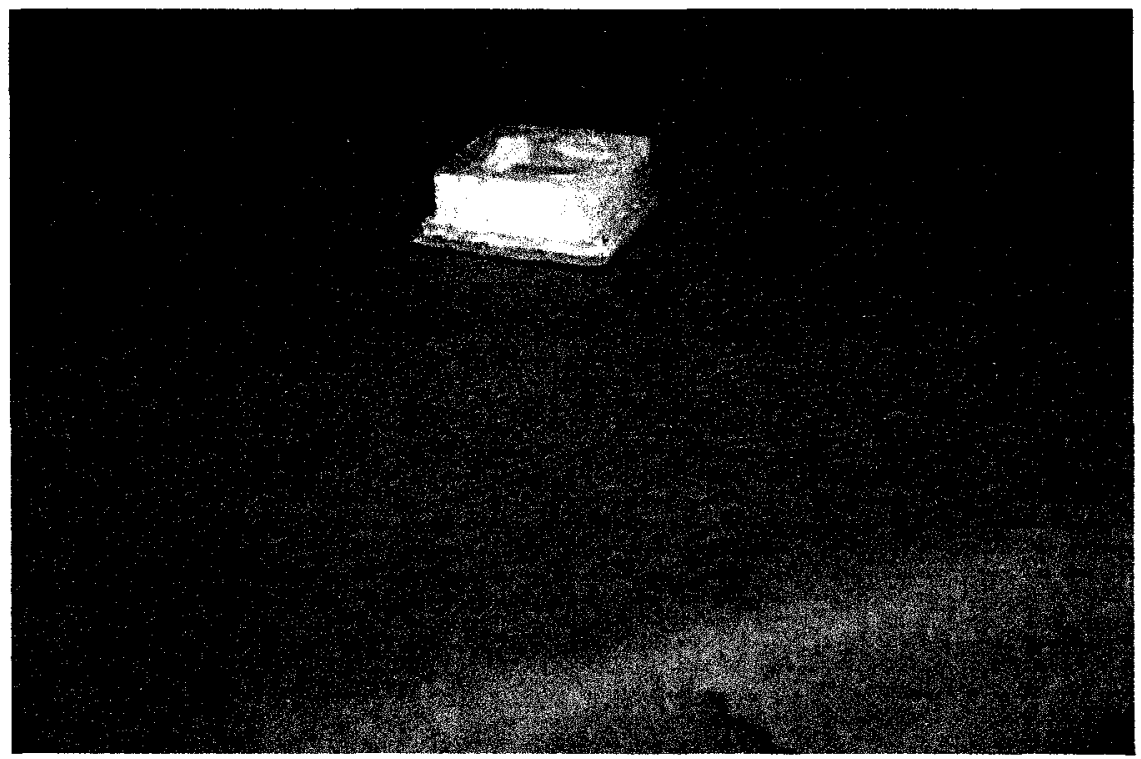

Fig 50: Zoom-in view of Hollow Pyramid Shape

The hollow pyramidal shape illustrated in Figure 50 uniquely displays the advantages and capabilities of the HRP system. This 4-layered sample was machined all around to allow a one-step indentation. The milling head was then positioned over the centre to 
machine out a 3-layer cavity directly in the middle of the prototype. Final examination of the piece revealed satisfactory side and top surface finishes.

Overall, the HRP system performed exceptionally well for a prototype design. The tolerances achieved by the system were in the competitive range of most commercial RP technologies in the market. Table 8 depicts a comparison chart that was shown in Chapter 1.2 of this report with an additional row to compare the HRP system with respect to other widely used commercial technologies.

Table 8: Technology Comparison For Accuracy Between HRP and RP

\begin{tabular}{|c|c|c|}
\hline RP Technology & Layer Thickness Range & $\begin{array}{c}\text { Accuracy } \\
\text { (Tolerance per linear inch) }\end{array}$ \\
\hline SLA & $0.004 "-0.030 "$ & $\begin{array}{c}0.1 \%-0.5 \% \text { of overall } \\
\text { dimension }\end{array}$ \\
\hline FDM & $0.010^{\prime \prime}-0.250 "$ & $0.001 "-0.050^{\prime \prime}$ \\
\hline SLS & $0.003 "-0.020 "$ & $0.005^{\prime \prime}-0.015^{\prime \prime}$ \\
\hline BPM & $0.003 "$ & $+/-0.004 "$ \\
\hline 3D Printing & $0.003 "-0.010 "$ & $+/-0.013 "$ \\
\hline LOM & $0.002 "-0.020$ & $+/-0.005^{\prime \prime}$ \\
\hline Hybrid RP System & $\mathbf{0 . 0 1 0 " - 0 . 0 6 0 "}$ & $+/-0.018 "$ \\
\hline
\end{tabular}

Table 8 illustrates the accuracy per inch of several RP technologies. It is evident from this table that despite the lack of robotic arms, carbon dioxide lasers and six-degree of freedom milling machines, the HRP system's accuracy is very comparable to other conventional systems. In particular, the FDM system was the building block design for the hybrid system. However, with the additional machining head on the hybrid system, it is clear from Table 8 that the tolerance for the HRP design is not as far spread as that for the FDM. Furthermore, the maximum average tolerance of the HRP system is significantly higher than that for the FDM. This competitive range is due to the extra machining head configuration, which is the foundation for the hybrid design. Each time a 
new layer is deposited (with the HRP system) it is laid down on a flat surface so as to reduce stair-step inaccuracies. Machining the perimeter and the surface after each layer ensures that accuracies are kept tight and high and it also ensures a cleaner and higher quality surface.

Table 9: Comparison Between The HRP And Commercial RP Systems under \$60,000 US [7]

\begin{tabular}{|c|c|c|c|c|}
\hline & $\begin{array}{c}\text { Actua } 2100 \text { (3D } \\
\text { Systems) }\end{array}$ & Genisys (Stratasys) & $\begin{array}{c}\text { Model Maker } \\
\text { (Sanders Prototype, } \\
\text { Inc.) }\end{array}$ & $\begin{array}{l}\text { Personal Modeller } \\
\text { (BPM Technology, } \\
\text { Inc.) }\end{array}$ \\
\hline $\begin{array}{l}\text { Maximum } \\
\text { model size }\end{array}$ & $\begin{array}{c}20 \text { in. } \times 8 \text { in. } \times 8 \\
\text { in. }\end{array}$ & 8 in. $x 8$ in. $\times 8$ in. & 6 in. $x 6$ in. $\times 6$ in. & 10 in. $\times 8$ in. $\times 6$ in. \\
\hline $\begin{array}{l}\text { Model } \\
\text { material }\end{array}$ & $\begin{array}{l}\text { Fragile plastic } \\
\text { polymer }\end{array}$ & $\begin{array}{l}\text { Durable plastic } \\
\text { polymer (can sand } \\
\text { it, paint it, and drill } \\
\text { it) }\end{array}$ & $\begin{array}{l}\text { Thermoplastic and wax } \\
\text { (for support during the } \\
\text { build process) }\end{array}$ & $\begin{array}{l}\text { Mixture of } \\
\text { thermoplastic polymers }\end{array}$ \\
\hline Technology & $\begin{array}{l}\text { Print head with } 96 \\
\text { jets delivers } \\
\text { thermo-polymer } \\
\text { material layer by } \\
\text { layer }\end{array}$ & $\begin{array}{l}\text { A 3-D printer with } \\
\text { material fed through } \\
\text { an extrusion head } \\
\text { layer by layer }\end{array}$ & $\begin{array}{l}\text { Liquid-to-solid inkjet } \\
\text { plotter deposits material } \\
\text { layer by layer }\end{array}$ & $\begin{array}{l}\text { Piezoelectric single jet } \\
\text { system delivers thermo- } \\
\text { plastic polymer material } \\
\text { layer by layer }\end{array}$ \\
\hline $\begin{array}{l}\text { Software } \\
\text { interface }\end{array}$ & $\begin{array}{l}\text { Allegro Software } \\
\text { and TCP/IP socket }\end{array}$ & AutoGen Software & $\begin{array}{l}\text { Prototype-Slicer } \\
\text { (operates with SLC, .stl, } \\
\text { AutoCAD DXF, HPGL } \\
\text { and OBJ files) }\end{array}$ & $\begin{array}{l}\text { Imports .stl or PRO/E } \\
\text { files }\end{array}$ \\
\hline $\begin{array}{l}\text { Material } \\
\text { supply }\end{array}$ & $\begin{array}{l}\text { Cartridge holding } \\
\text { thermo-polymer } \\
\text { material }\end{array}$ & $\begin{array}{l}\text { Cassettes holding } \\
\text { wafers of plastic } \\
\text { polymer }\end{array}$ & --- & $\begin{array}{l}\text { Plastic beads poured } \\
\text { into the machine } \\
\text { becomes molten } \\
\text { thermo- plastic }\end{array}$ \\
\hline Size & $\begin{array}{l}54 \text { in (w) } \times 30 \text { in } \\
(d) \times 44 \text { in (h) }\end{array}$ & $\begin{array}{l}36 \text { in (w) x } 29 \text { in (d) } \\
\times 32 \text { in (h) }\end{array}$ & $\begin{array}{l}20.5 \text { in }(w) \times 17.5 \text { in (d) } \\
\times 23 \text { in (h) }\end{array}$ & $\begin{array}{l}20.5 \text { in (w) } \times 24 \text { in (d) } \times \\
56 \text { in (h) }\end{array}$ \\
\hline Weight & 700 pounds & 184 pounds & 71 pounds & 250 pounds \\
\hline Accuracy & - & $\begin{array}{l}\text { Within .356 mm } \\
(.014 \text { in. })\end{array}$ & $\begin{array}{l}\text { Within } 0.05 \mathrm{~mm}(0.002 \\
\text { in.) }\end{array}$ & $\begin{array}{l}+/-0.51 \mathrm{~mm} / \mathrm{mm} \\
\left(0.020^{\prime \prime}\right) \text { under } 51 \mathrm{~mm}\end{array}$ \\
\hline Price (US \$) & $\$ 60,000$ & $\$ 50,000$ & $\$ 55,000$ & $\$ 35,000$ \\
\hline
\end{tabular}

Table 9 shows some of the most widely used and accepted RP machines in North America under $\$ 60,000$. These RP machines are built by well known RP manufactures such as 3D Systems, Stratasys, Sanders Prototype Inc and BPM technology Inc. The comparison criterion of most interest for this report is that of dimensional accuracy and the difference between the accuracies of these machines versus those achieved by the 
hybrid RP. Unfortunately, there was no empirical data based on accuracy for the "Actua 2100 ", which was built by 3D Systems. Therefore, emphasis will be placed on the latter three machines. Referring to Table 9, the "Genisys" (figure 51) built by Stratasys has a published tolerance of $0.014 "$ " The HRP system has a documented tolerance of 0.018 ". Regardless of the fact that the "Genisys" was built by a world-class RP manufacturer with technology, manpower and capital at its disposal, it only surpassed the accuracy of the HRP system by 0.004 ". The next commercially available RP machine in the market is the "Personal Modeller" built BPM Technology Inc. Referring to table 9, it can be seen that for parts smaller than $51 \mathrm{~mm}(2.009$ "), the published accuracy for the "Personal Modeller" is $0.020^{\prime \prime}$. When compared with the HRP system' accuracy of 0.018 ", it is evident that with respect to accuracy, the HRP has a clear advantage over this commercially produced RP machine. Finally, with respect to the "Model Maker" built by Sanders Prototype Inc., which as an accuracy value of 0.002 ", it is clear that, the HRP system is no competition. Nevertheless, ranking in third place $(0.004$ " behind second place) in a comparison between three widely used and accepted RP machines in the world is quite an achievement. The HRP design has proven that when compared directly with the leading RP machines in the world, it is very competitive with respect to accuracy.

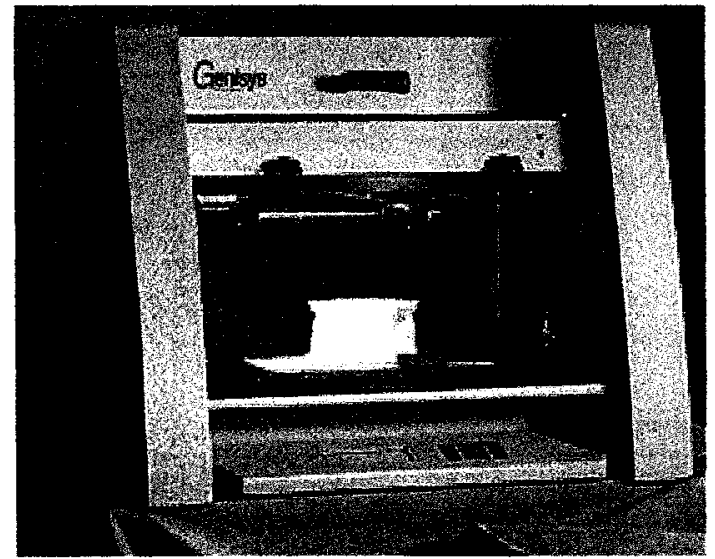

Fig 51: Genisys Commercial System

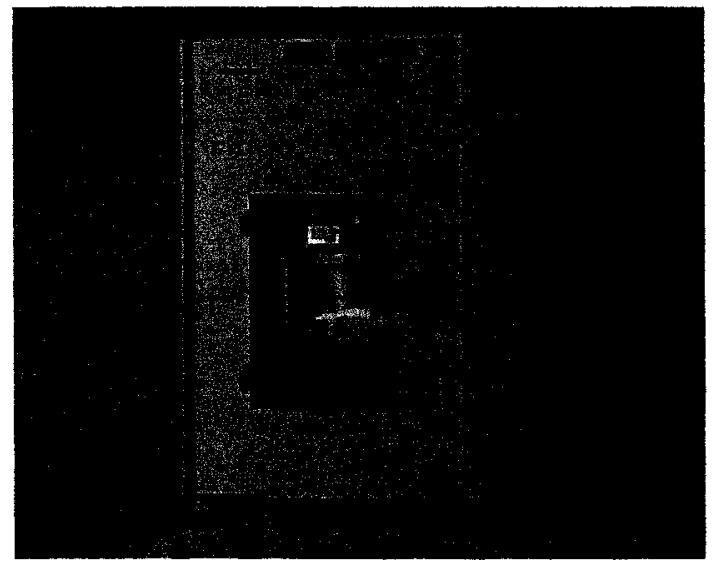

Fig 52: FDM 1650 Commercial System 
Table 10 shows the most widely used commercial RP machines over $\$ 100,000$ US in value. The criterion of most relevance to this report is that of accuracy. The drastic increase in price of this class of machines only constitutes an increase in accuracy of 0.010 " with respect to the HRP. Again, it is evident that the HRP system is very aggressive and competitive with respect to popular commercial systems.

Table 10: Comparison Between The HRP And Commercial RP Systems over $\$ 100,000$ US [8]

\begin{tabular}{|c|c|c|c|c|c|}
\hline & LOM & FDM & SLA & SLS & SGC \\
\hline Model & LOM 1015 & $\begin{array}{l}\text { FDM } 1650 \\
\text { (Figure } 52 \text { ) }\end{array}$ & SLA-250 & $\begin{array}{l}\text { Sinterstation } \\
2000\end{array}$ & $\begin{array}{l}\text { Solider } \\
\text { SGC5600 }\end{array}$ \\
\hline Company & Helisys & Stratasys & 3D Systems & DTM Corp. & Cubital \\
\hline $\begin{array}{c}\text { Machine } \\
\text { price }\end{array}$ & $-\$ 120,000$ & $\sim \$ 100,000$ & $\sim \$ 100,000$ & $\sim \$ 300,000$ & $\$ 470,000$ \\
\hline $\begin{array}{c}\text { Materials } \\
\text { used }\end{array}$ & $\begin{array}{l}\text { Adhesive } \\
\text { coated paper }\end{array}$ & $\begin{array}{c}\text { ABS, } \\
\text { Casting wax, } \\
\text { elastomers, } \\
\text { medical } \\
\text { ABS }\end{array}$ & $\begin{array}{c}\text { Photo- } \\
\text { sensitive } \\
\text { epoxy resin }\end{array}$ & $\begin{array}{c}\text { Nylons, } \\
\text { Powdered } \\
\text { steel, sand, } \\
\text { Polycarbonate }\end{array}$ & $\begin{array}{l}\text { Photopolymer } \\
\text { acrylate resins }\end{array}$ \\
\hline $\begin{array}{l}\text { Material } \\
\text { costs }\end{array}$ & $\$ 200 /$ roll & Varies & \$\$ & $\$ 25-32 / \mathrm{lb}$ & $\$ 35 / \mathrm{lb}$ \\
\hline $\begin{array}{l}\text { Accuracy } \\
\text { (in.) }\end{array}$ & 0.010 & 0.007 & 0.009 & $\mathbf{N} / \mathbf{A}$ & 0.008 \\
\hline $\begin{array}{l}\text { Max part } \\
\text { size }(\mathrm{cm})\end{array}$ & $38 \times 25 \times 36$ & $25 \times 25 \times 25$ & $25 \times 25 \times 25$ & $\begin{array}{c}30 \text { Dia. } \times 38 \\
\text { high }\end{array}$ & $50 \times 35 \times 50$ \\
\hline $\begin{array}{c}\text { Layer } \\
\text { thickness } \\
(\mathbf{m m})\end{array}$ & $0.05-0.38$ & $0.05-0.762$ & 0.15 & $\begin{array}{c}0.0762- \\
0.508\end{array}$ & $0.0762-0.203$ \\
\hline Software & LOMSlice & QuickSlice & Maestro/JR & Proprietary & $\begin{array}{c}\text { Data Front } \\
\text { End }\end{array}$ \\
\hline $\begin{array}{l}\text { File } \\
\text { formats }\end{array}$ & STL & STL & STL, SLC & STL & $\begin{array}{l}\text { STL, DXF, } \\
\text { VDA, EFL }\end{array}$ \\
\hline
\end{tabular}


To further enforce the fact that the machining head attachment was the main factor that improved overall dimensional accuracy and surface quality, Table 11 illustrates the measurements of a one layered-square test piece with and without machining. Five trials were carried out in which the test piece was first created strictly by depositing the wax on the working surface and having its measurements recorded. Next, the machining phase of the system was re-engaged to machine the surface and perimeter of the test piece and its measurements were also recorded.

Table 11: Prototyping With and Without Machining

\begin{tabular}{|c|c|c|c|c|c|c|c|}
\hline \multirow{2}{*}{\begin{tabular}{|l} 
\\
Shape \\
\end{tabular}} & \multirow[b]{2}{*}{ Run } & \multicolumn{2}{|c|}{$\begin{array}{l}\text { Required } \\
\text { Size (Inches) }\end{array}$} & \multicolumn{2}{|c|}{$\begin{array}{c}\text { Exact Size } \\
\text { Without Machining }\end{array}$} & \multirow{2}{*}{\begin{tabular}{|l|} 
Tolerance \\
For Length \\
\end{tabular}} & \multirow{2}{*}{$\begin{array}{l}\text { Tolerance } \\
\text { For Width } \\
\end{array}$} \\
\hline & & Length & Width & Length & Width & & \\
\hline \multirow[t]{6}{*}{ Square } & 1 & 1.000 & 1.000 & 1.238 & 1.216 & 0.238 & 0.216 \\
\hline & 2 & 1.000 & 1.000 & 1.196 & 1.216 & 0.196 & 0.216 \\
\hline & 3 & 1.000 & 1.000 & 1.221 & 1.265 & 0.221 & 0.265 \\
\hline & 4 & 0.750 & 0.750 & 0.975 & 1.010 & 0.225 & 0.260 \\
\hline & \multirow[t]{2}{*}{5} & \multirow[t]{2}{*}{0.750} & \multirow[t]{2}{*}{0.750} & \multirow[t]{2}{*}{1.020} & 0.995 & 0.270 & 0.245 \\
\hline & & & & & $\begin{array}{r}\text { AVG: } \\
\text { Total AVG: } \\
\end{array}$ & $\begin{array}{l}0.230 \\
0.235 \\
\end{array}$ & 0.240 \\
\hline & & \multicolumn{2}{|c|}{$\begin{array}{c}\text { Required } \\
\text { Size (Inches) }\end{array}$} & \multicolumn{2}{|c|}{$\begin{array}{c}\text { Exact Size } \\
\text { With Machining }\end{array}$} & Tolerance & Tolerance \\
\hline Shape & Run & Length & Width & Length & Width & For Length & For Width \\
\hline \multirow[t]{6}{*}{ Square } & 1 & 1.000 & 1.000 & 1.013 & 0.978 & 0.013 & 0.022 \\
\hline & 2 & 1.000 & 1.000 & 1.016 & 1.020 & 0.016 & 0.020 \\
\hline & 3 & 1.000 & 1.000 & 0.980 & 1.045 & 0.020 & 0.045 \\
\hline & 4 & 0.750 & 0.750 & 0.730 & 0.758 & 0.020 & 0.008 \\
\hline & \multirow[t]{2}{*}{5} & \multirow[t]{2}{*}{0.750} & \multirow[t]{2}{*}{0.750} & \multirow[t]{2}{*}{0.758} & 0.769 & 0.008 & 0.019 \\
\hline & & & & & $\begin{array}{r}\text { AVG: } \\
\text { Total AVG: }\end{array}$ & $\begin{array}{l}0.015 \\
0.019\end{array}$ & 0.023 \\
\hline
\end{tabular}

Reviewing Table 11, it is clear that prototyping without machining gave very unacceptable and undesirable results with accuracies exceeding a quarter of an inch (0.250"). In a manufacturing environment, these tolerances would not be acceptable. However, once the program for the machining portion of the system was re-engaged, the 
tolerances were returned to their acceptable and normal parameters. This quick and clear demonstration shows the advantage in having a hybrid system when tight tolerances are an issue. In summary, every existing conventional RP machine in the market has the potential to become a HRP. By retrofitting the conservative RP systems with a machining head and custom calibrating the software for each particular case, the new hybrid system can achieve accuracies and surface qualities that were unattainable before the modifications. 


\section{CHAPTER 4-CONCLUSIONS AND RECOMMENDATIONS}

\subsection{Conclusion}

An existing rapid prototyping machine was modified to become a Hybrid Rapid Prototyping (HRP) system. Software was written using Visual Basic to create a user friendly interface in order to allow the operator to choose amongst a number of simple geometric shapes available to rapidly produce prototype parts. Furthermore, optimization experiments were carried out to determine the most favourable parameters with respect to nozzle diameter, gap distance, feed rate and the stepper motor pump rate in order to allow an optimal environment for prototype production. Once these parameters were discovered, further experimentation was conducted to assess the system's accuracy for manufacturing three-dimensional parts. The experiments performed suggested tolerances of 0.018 " for cubical shapes and tolerances of $0.022 "$ for cylindrical shapes. These tolerances are well within the competitive range of commercially available RP machines in the market to date. Furthermore, experiments dealing with the effectiveness of a milling head attachment revealed that prototyping without machining produced tolerances of roughly 0.250 " and prototyping with machining showed tolerances of 0.019". Therefore, prototyping with machining presented results that were approximately 13 times better than those results obtained by just deposition prototyping. The proposed hybrid prototyping system was modified with a machining apparatus to perform both deposition and machining in a single station and thus it is expected that other standard systems in the future can also be retrofitted to accommodate a machining station in order to improve accuracy and overall quality. 


\subsection{Future Recommendations}

Although the HRP system is fully operational, there are however key areas where improvements could be made. One such area is apparent when depositing the second layer on to the first layer. It was stated in this report that a candle was placed under the table to allow the first layer to heat up slightly so that a strong cohesive bond could be produced between each layer to securely hold the part into place. However, one candle cannot obviously distribute an even heat supply to the surface area of the part. Secondly, the heat was being generated from the bottom, whereas the bond would be taking place on the face of the layer. Therefore, one quick suggestion could be to design a heating coil system to be placed beside the extrusion heat so that once the machining phase has completed its program, the heating coils could then be turned on to allow an even supply of heat to the surface of the layer before the deposition of the second layer. Conversely, heat needed to be taken away after the deposition of each layer before the machining phase could begin. Therefore, another recommendation could be the addition of a small battery operated cooling fan to be placed above the table to allow cooling of the part so as to reduce production time and also as a means of blowing debris out of the way once machining has begun.

Another area for future research and effort could be directed towards the use of the original single pump screw design system in combination with the wax extrusion system. The extrusion head could be used with a material slightly stronger than wax (such as polyethenol plastic) in conjunction with the deposition of the wax and the machining head to produce very complex prototype parts. The system would thus consist of three 
independently controlled stations to deliver wax, plastic and machining capabilities to generate prototypes.

In retrospect, the one advantage that the original system has over this new hybrid design is the fact that it had a large reservoir of molten wax awaiting production. The wax extrusion head is only capable of storing about three layers worth of wax before it needs refilling. Furthermore, each time the cylinder is refilled with liquid wax, a given amount of time is required for the wax to solidify and then to reach a steady state temperature of $53^{\circ} \mathrm{C}$. The obvious solution for this deficiency is that a new larger cylinder with a customized piston be designed and manufactured. 


\section{References}

[1] Kochan, Detlef and Zhaouhui, Du, "Rapid Prototyping issues in the $21^{\text {st }}$ Century," Journal of Computers in Industry, vol. 39, pp. 3-10, 1999.

[2] Yan, X. and Gu, P., "A Review Of Rapid Prototyping Technologies and systems," Journal of Computer Aided Design, vol. 28, pp. 307-318, 1996.

[3] Imaging Technology Group, Online Product Description, http://www.itg.uiuc.edu/vmil/printing.htm (Apr. 17, 2003).

[4] Hur, Junghoon. "Development of a hybrid rapid prototyping system - hardware and software," Proceedings of the third world congress on intelligent manufacturing processes \& systems. Cambridge, Massachusetts, USA, June 28-30, 2000.

[5] Jeng JY, Lin M.C., "Mold fabrication and modification using hybrid processes of selective laser cladding and milling," Journal of Materials Processing Technology, vol. 110, pp. 98-103, 2001.

[6] Chen, Y.H. and Song, Y, "The development of a layer based machining system," Journal of Computer Aided Design, vol. 33, pp. 331-342, 2001.

[7] San Diego Supercomputer Centre, Online Description, http://www.sdsc.edu/tmf/Research/lowcost.html (May 28, 2003).

[8] Penn State Learning Factory Rapid Prototyping, Online Description, http://www.me.psu.edu/lamancusa/rapidpro/tutorial/table.htm (May 28, 2003).

[9] Anitha, R. and Radhakrishnan, P., "Critical parameters influencing the quality of prototypes in Fused Deposition Modelling," Journal of Materials Processing Technology, vol. 118, pp. 385-388, 2001. 
[10] Choi, S.H. and Samavedam, S., "Modelling and optimization of Rapid Prototyping," Journal of Computers in Industry, vol. 47, pp. 39-53, 2002.

[11] Cormier, Denis and Taylor, James, “A process for solvent welded rapid prototype tooling," Robotics and Computer Integrated Manufacturing, vol. 17, pp. 151-157, 2001.

[12] King, D. and Tansey, T., "Alternative materials for rapid tooling," Journal of Materials Processing Technology, vol.121, pp. 313-317, 2002.

[13] Lee, Kwan H., "Direct integration of reverse engineering and rapid prototyping," Journal of Computers \& Industrial Engineering, vol. 38, pp. 21-38, 2002.

[14] Li, L. and Peng, X., "A reverse engineering system for rapid manufacturing of complex objects," Robotics and Computer Integrated Manufacturing, vol. 18, pp. 53-67, 2002.

[15] Rosochowski, A. and Matuszak, A., "Rapid tooling: The state of the art," Journal of Materials Processing Technology, vol. 106, pp. 191-198, 2002.

[16] Wang, Yufei and Duarte, Jose Pinto, "Automatic generation and fabrication of designs," Journal of Automation in Construction, vol. 11, pp. 291-302, 2002. 


\section{APPENDIX 1}




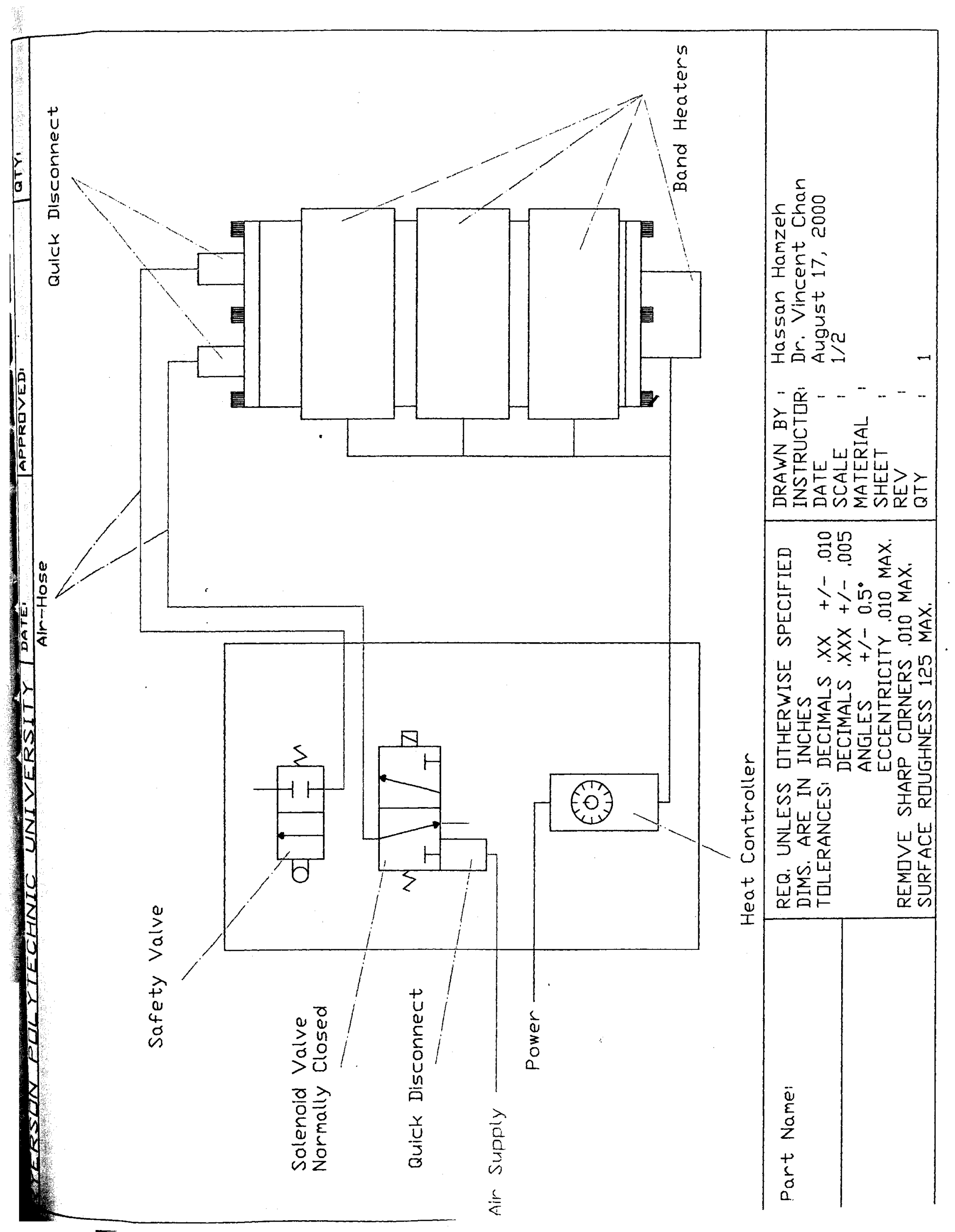

Reproduced with permission of the copyright owner. Further reproduction prohibited without permission. 


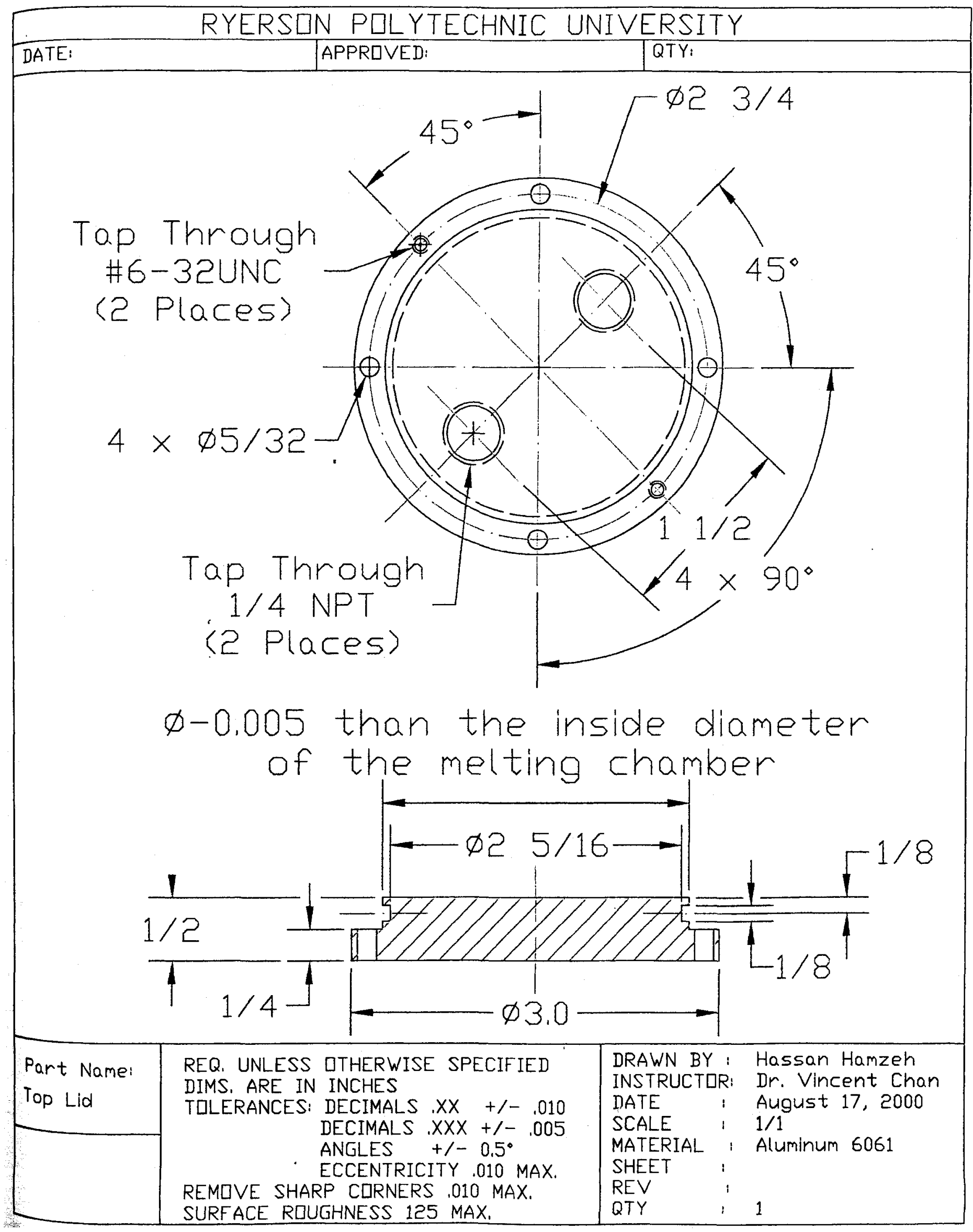




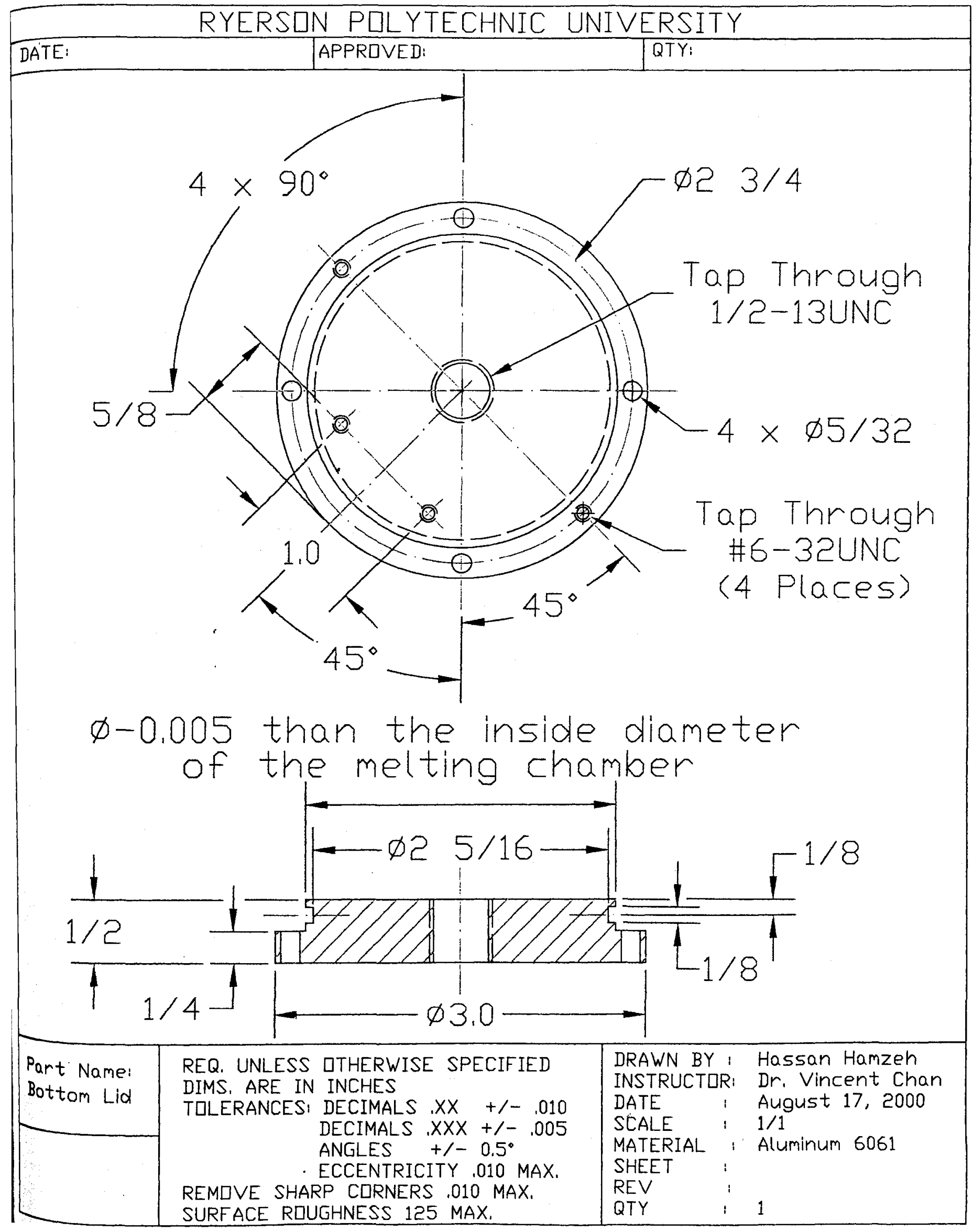




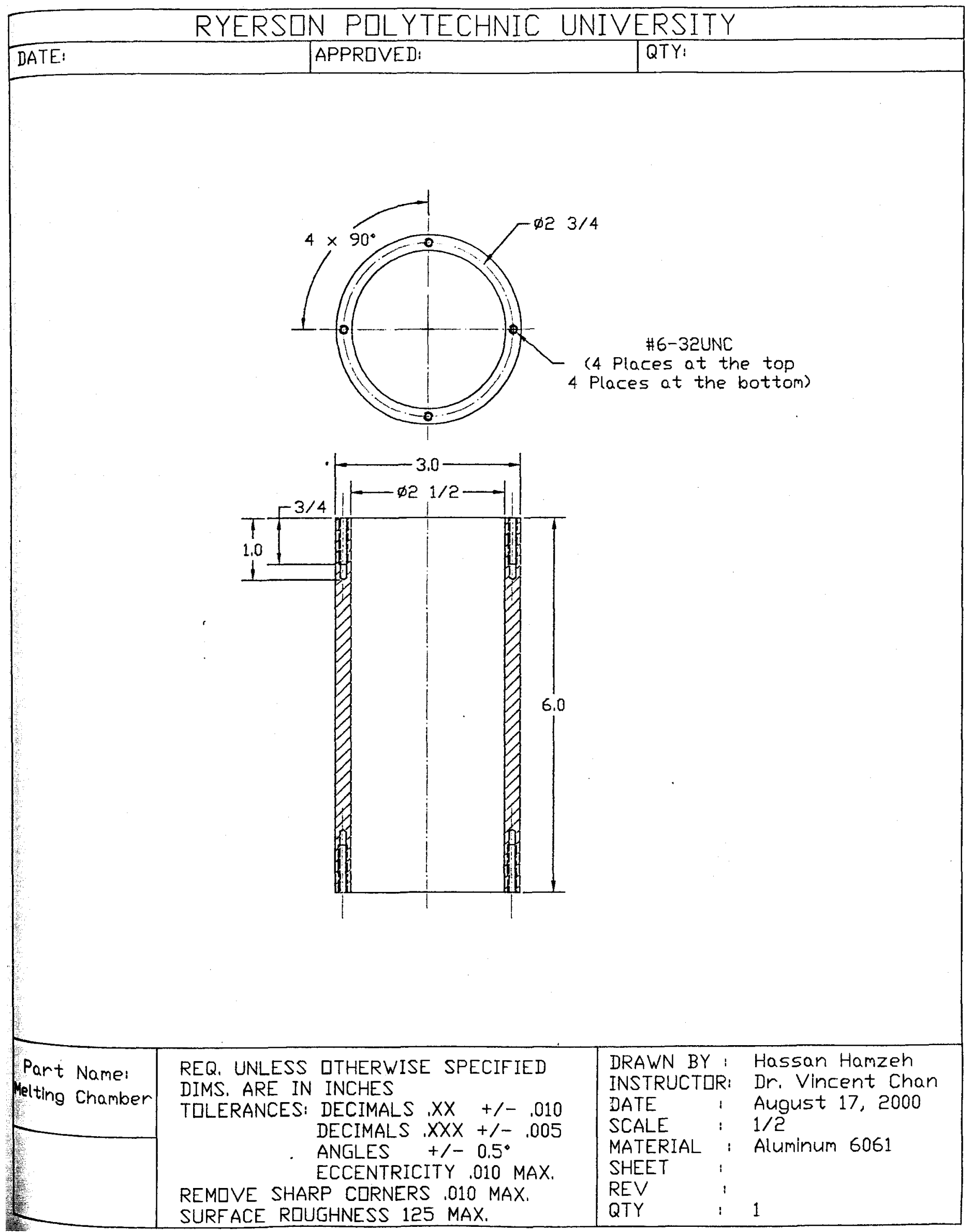




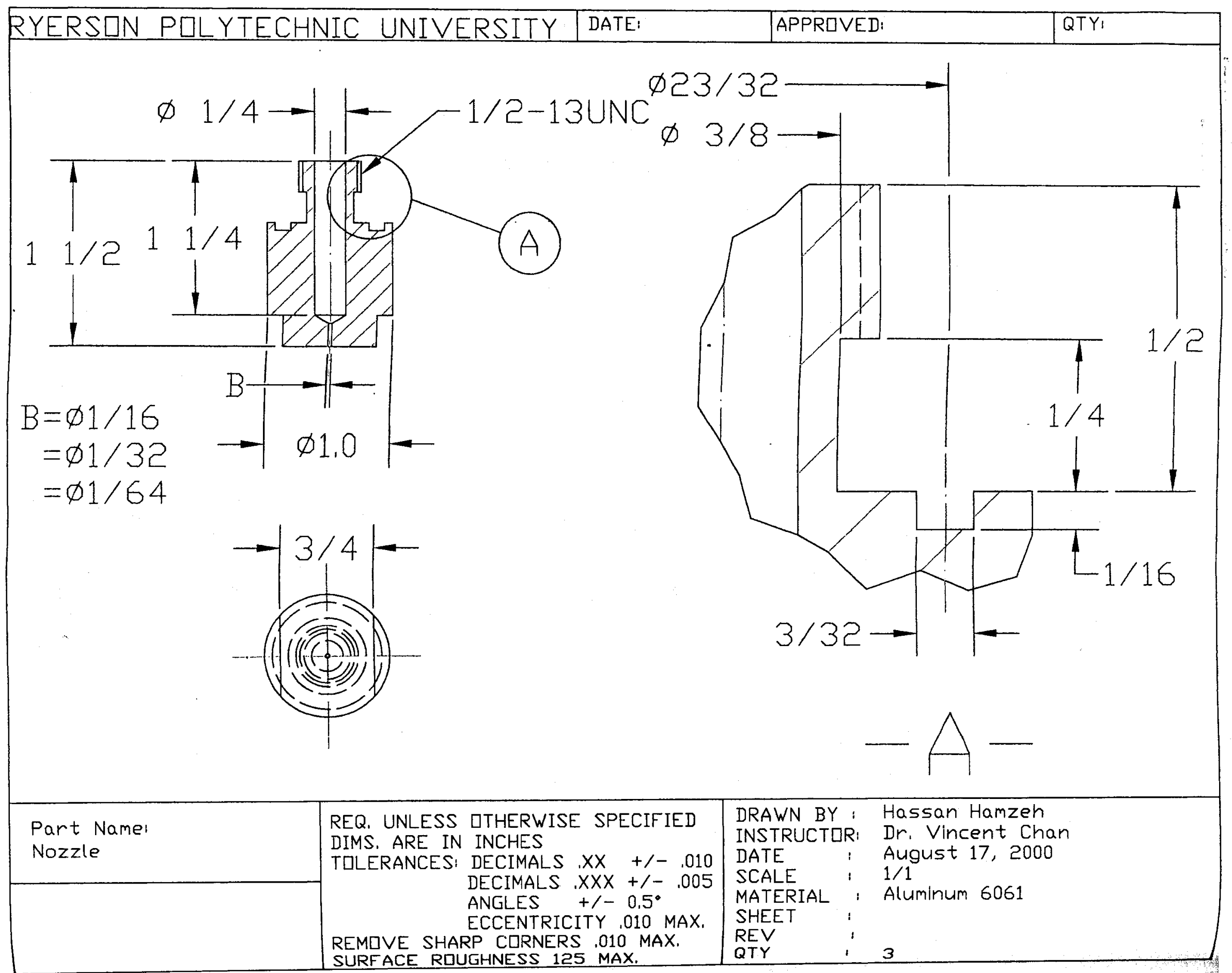




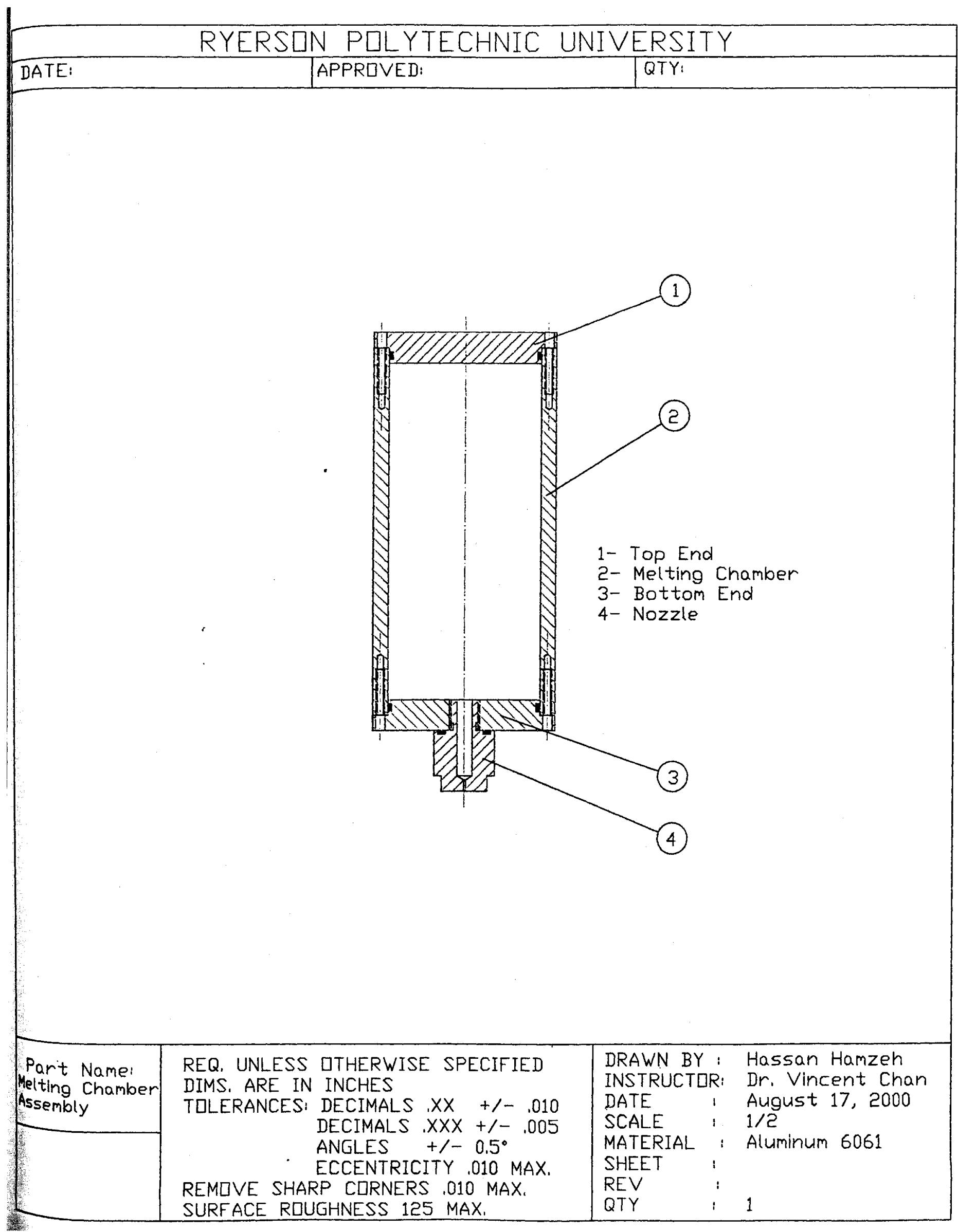




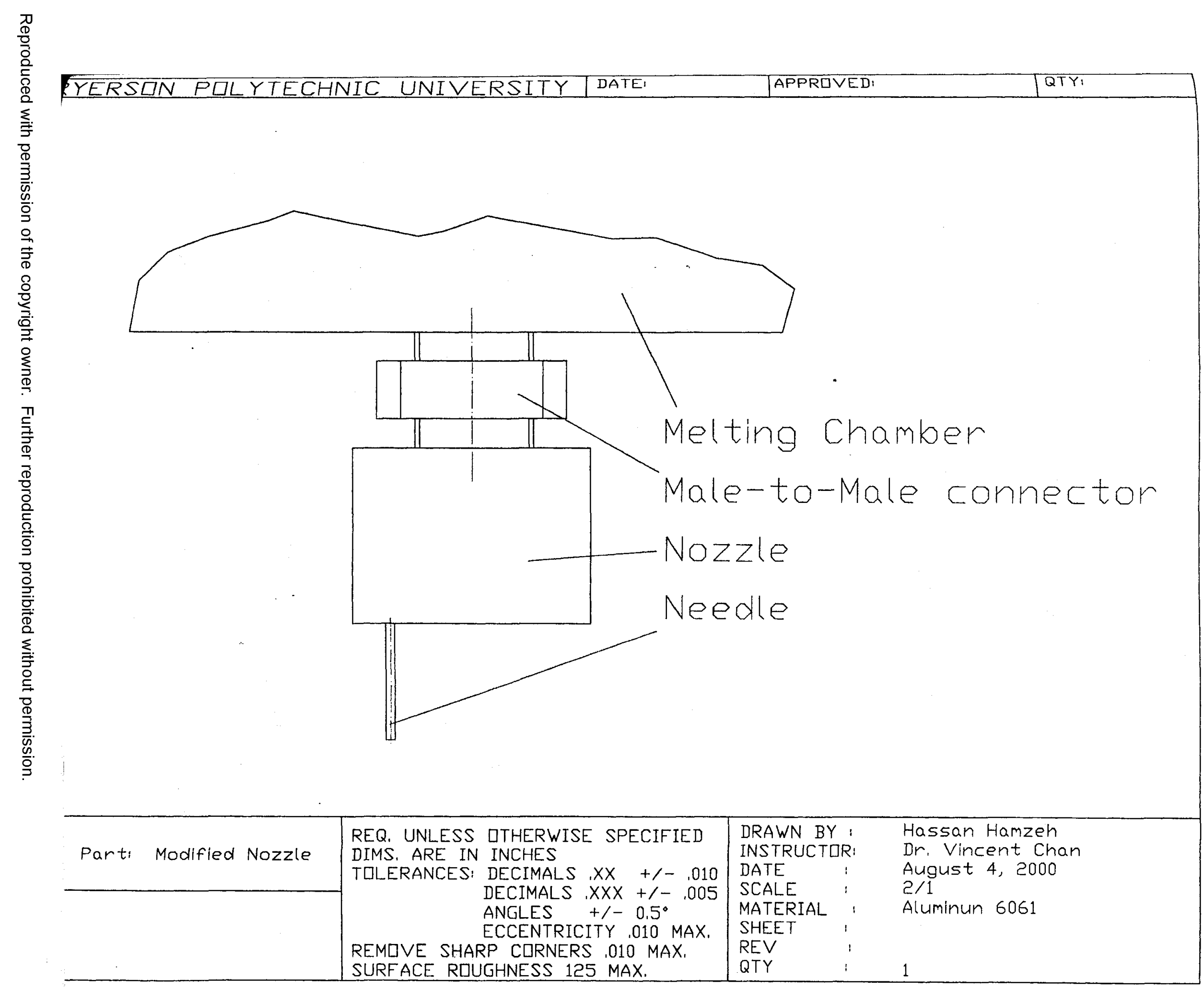




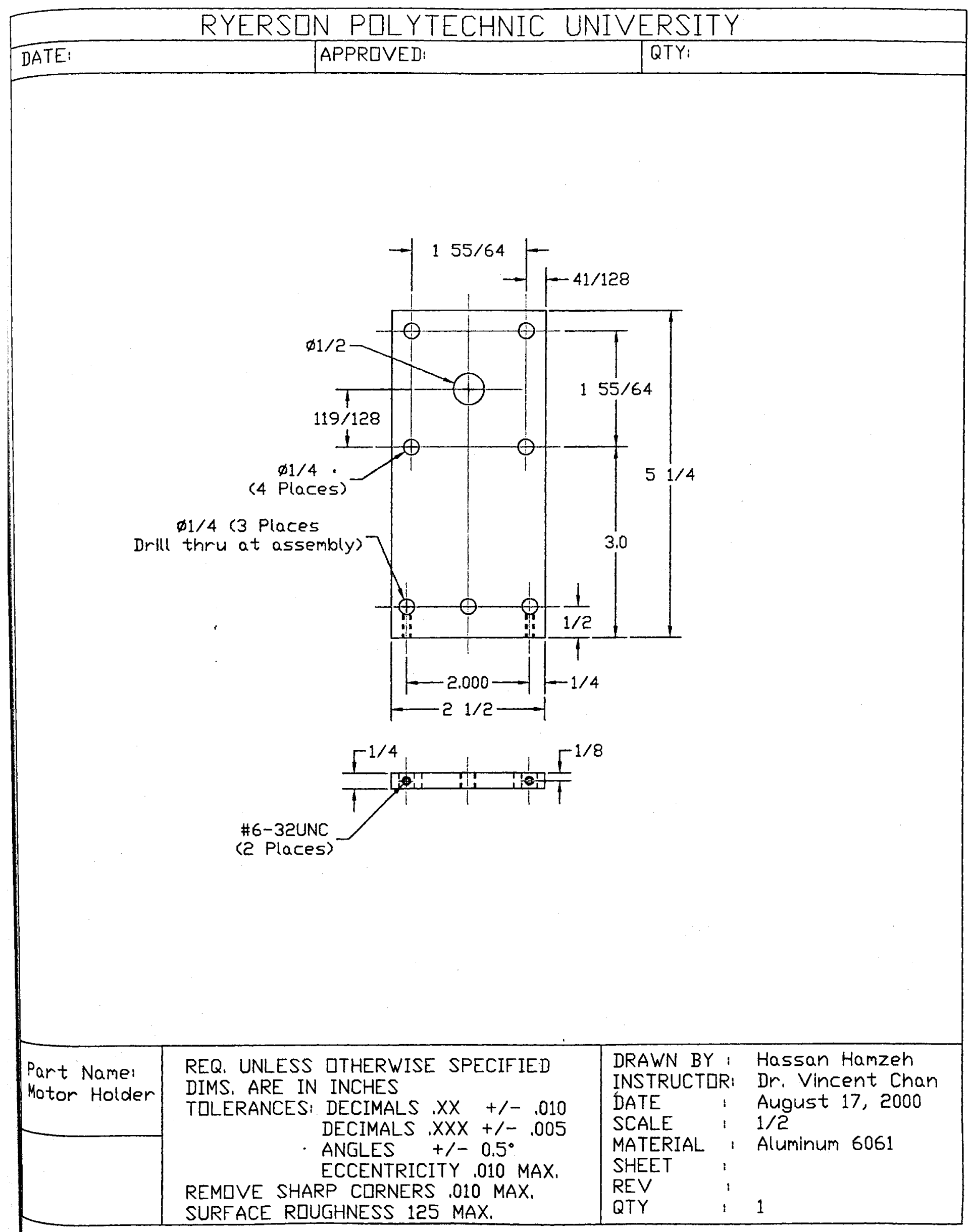




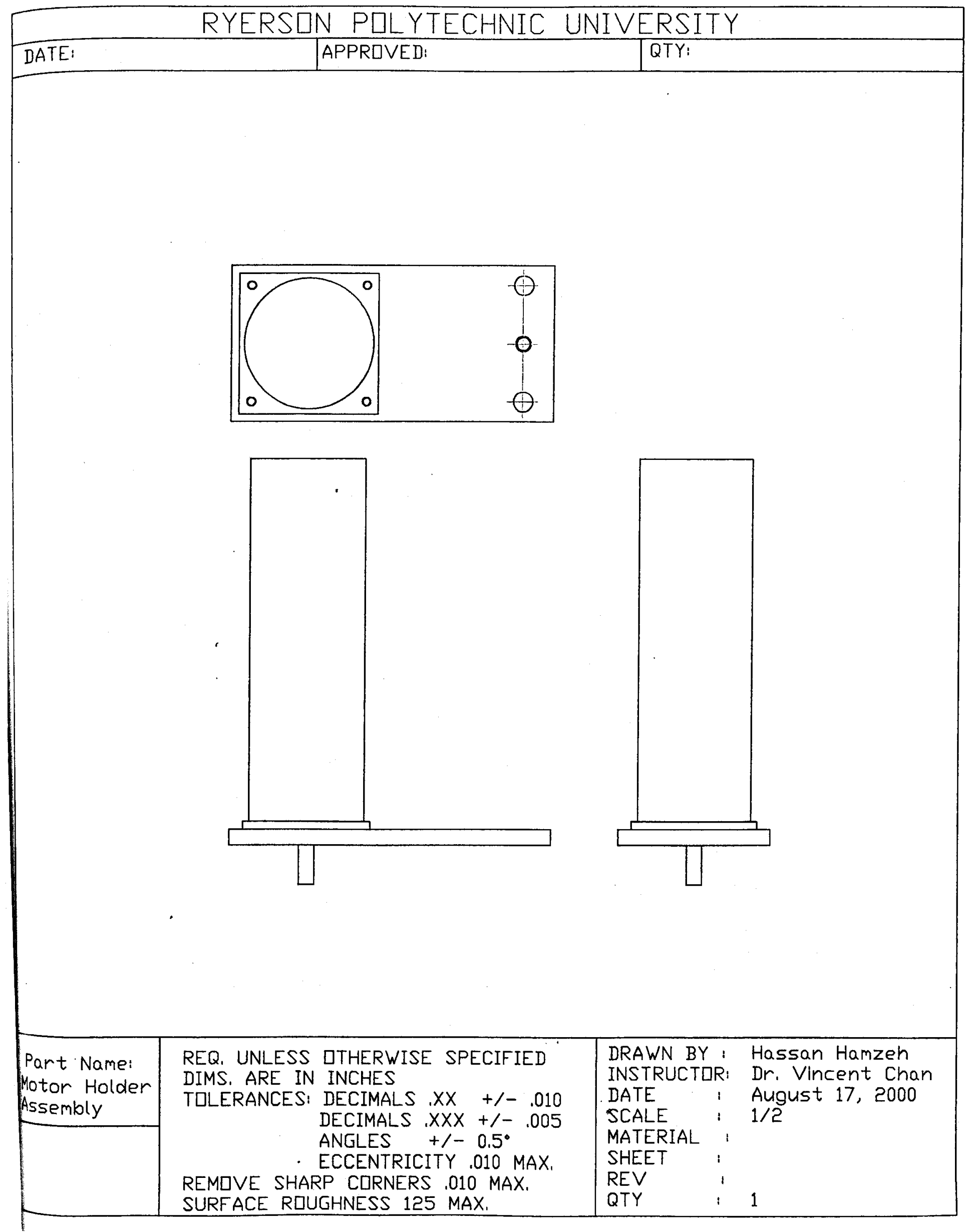




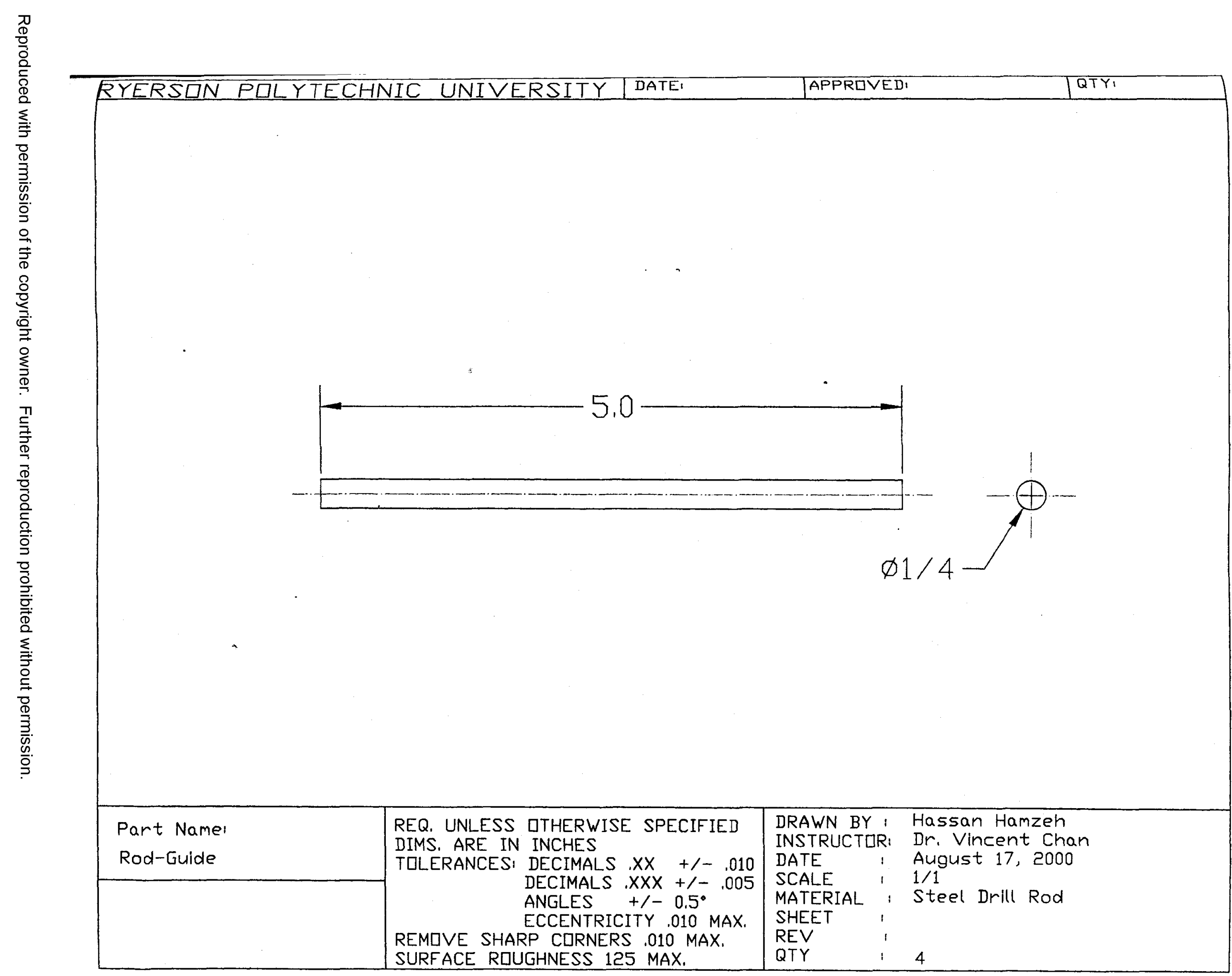




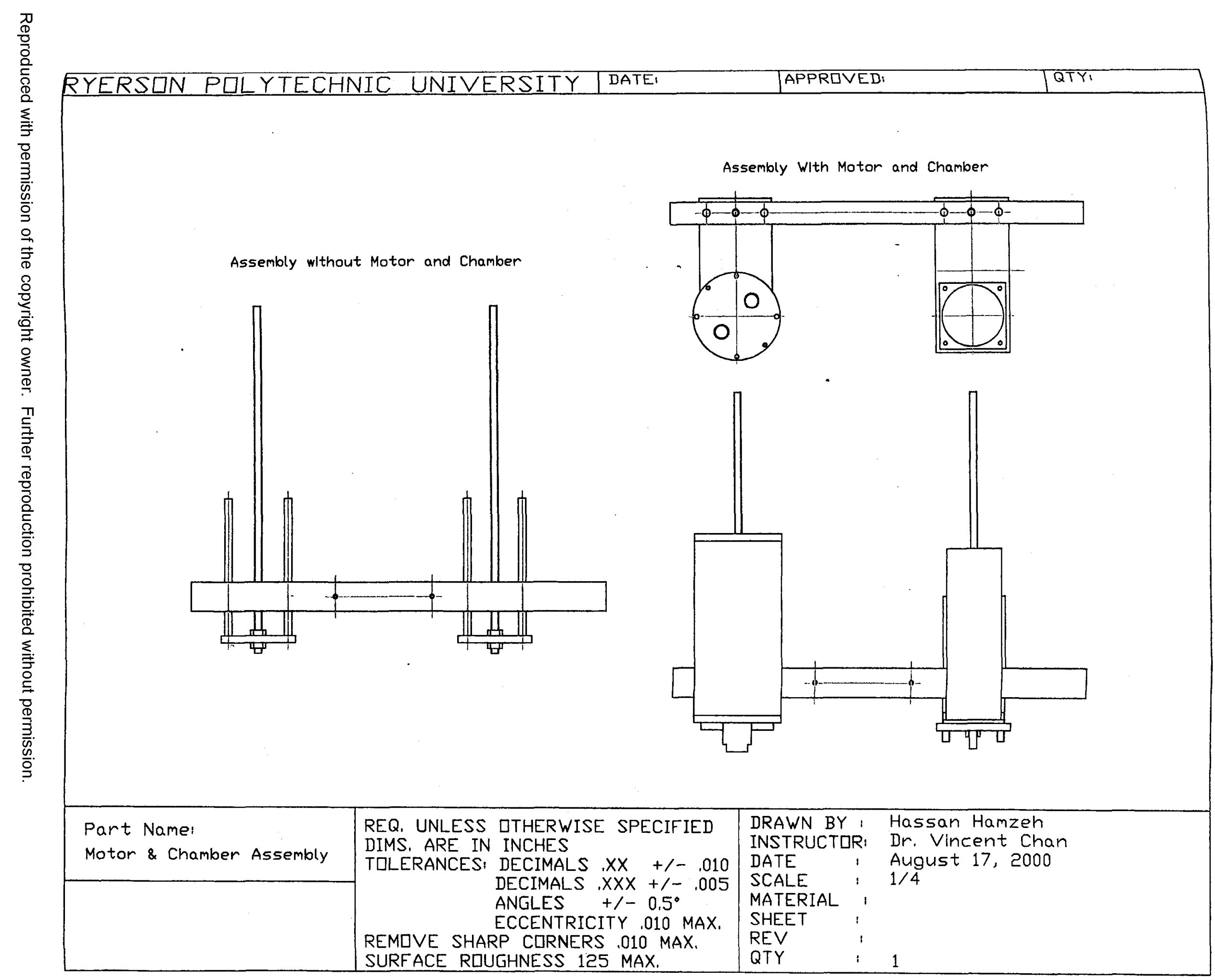




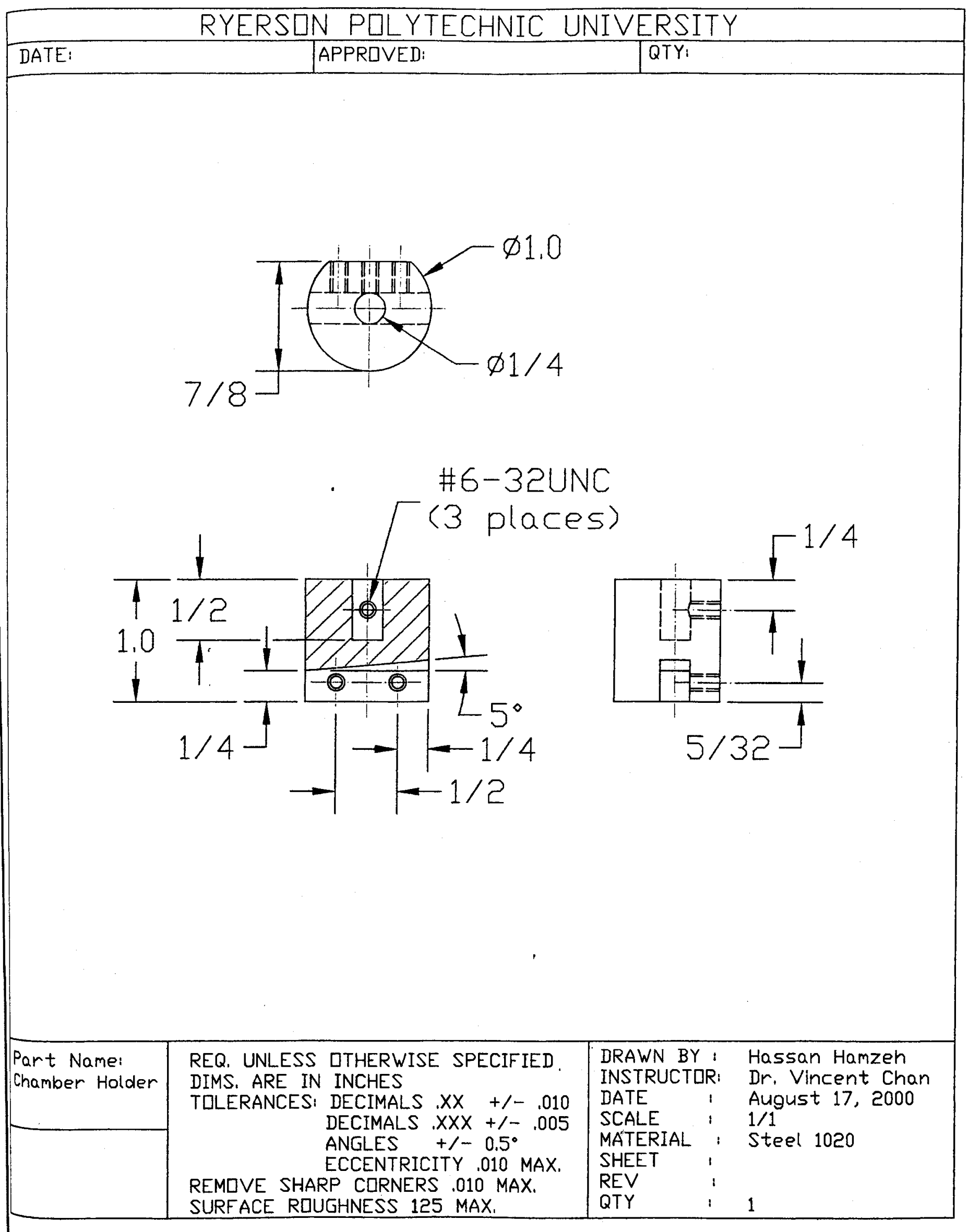




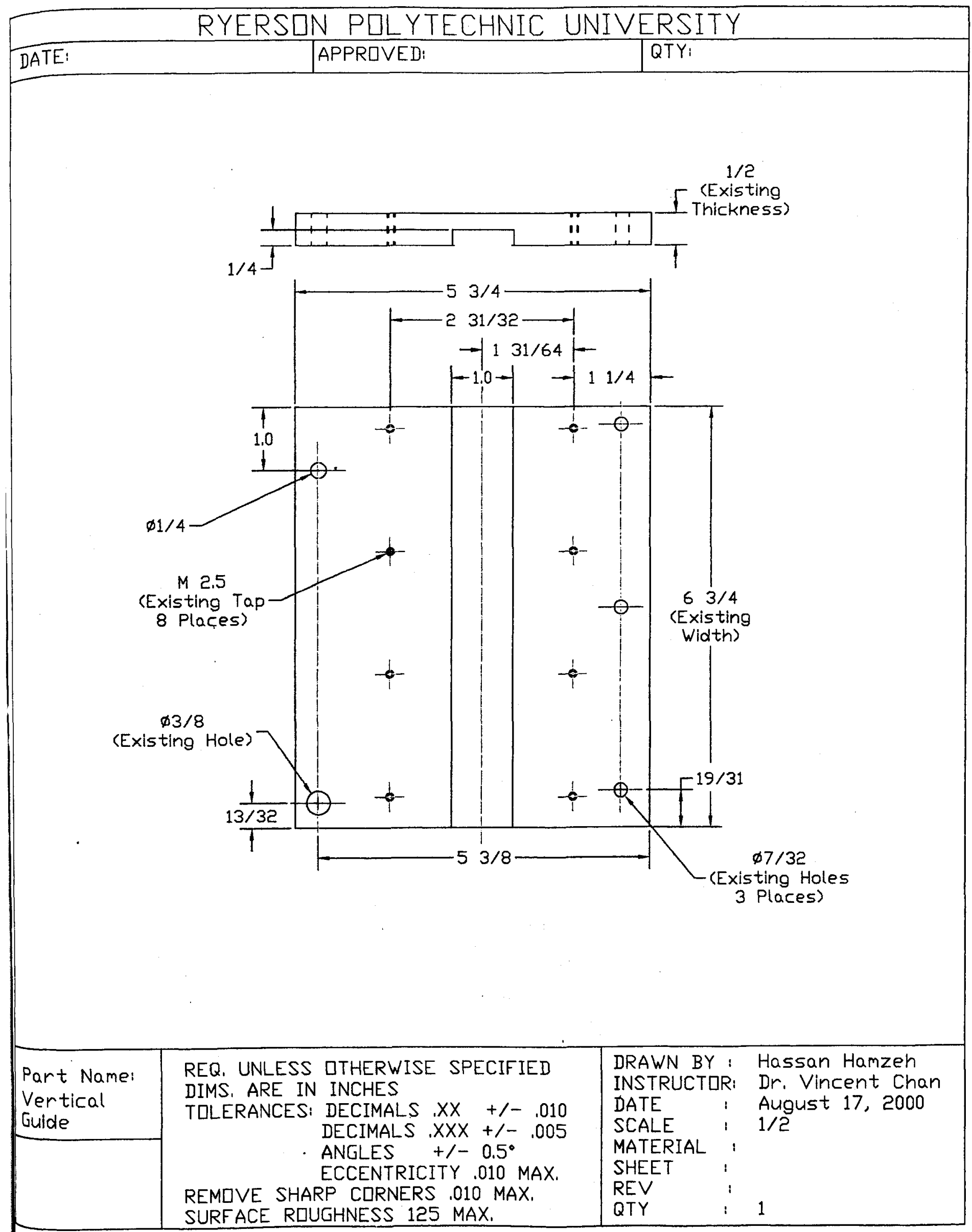




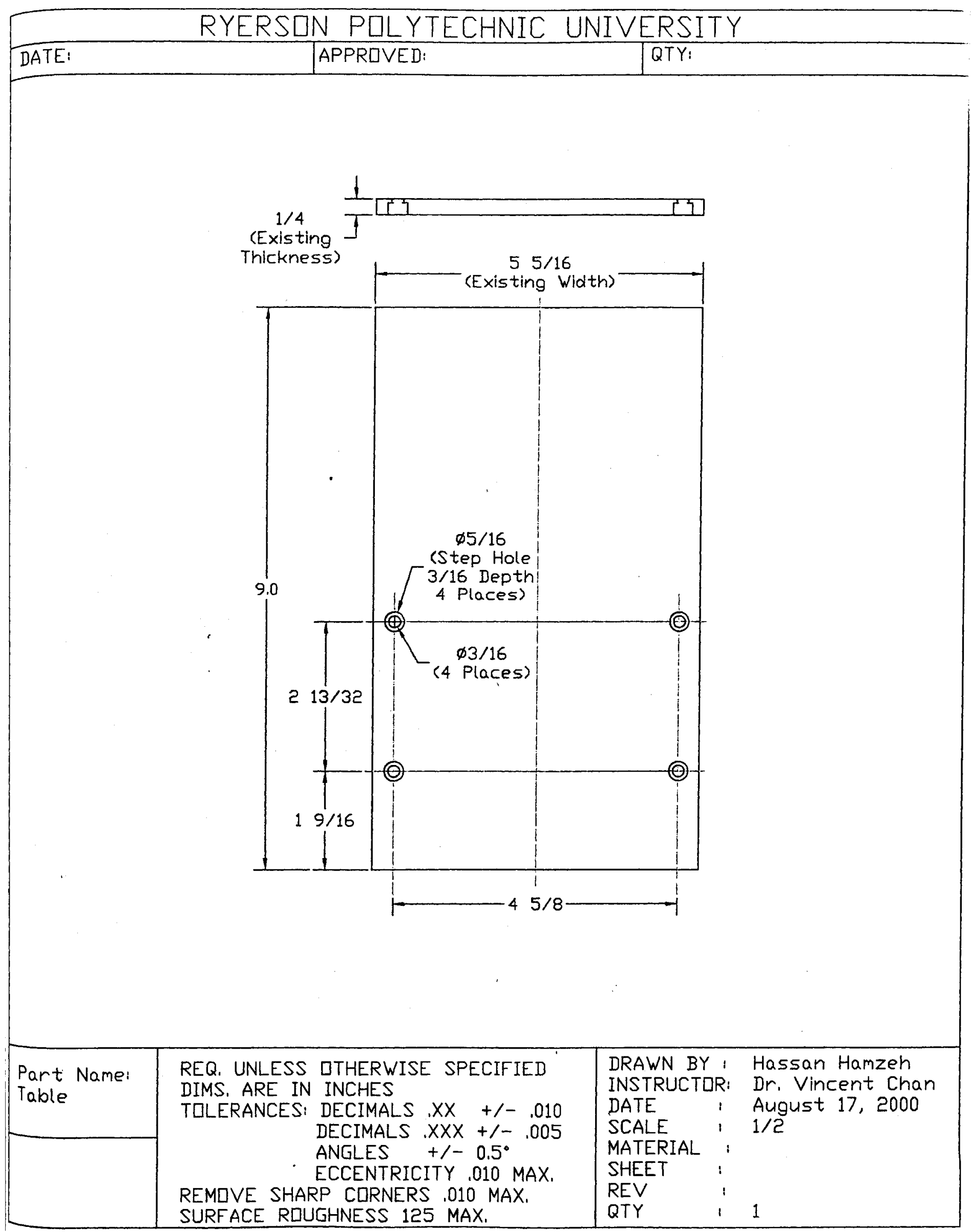




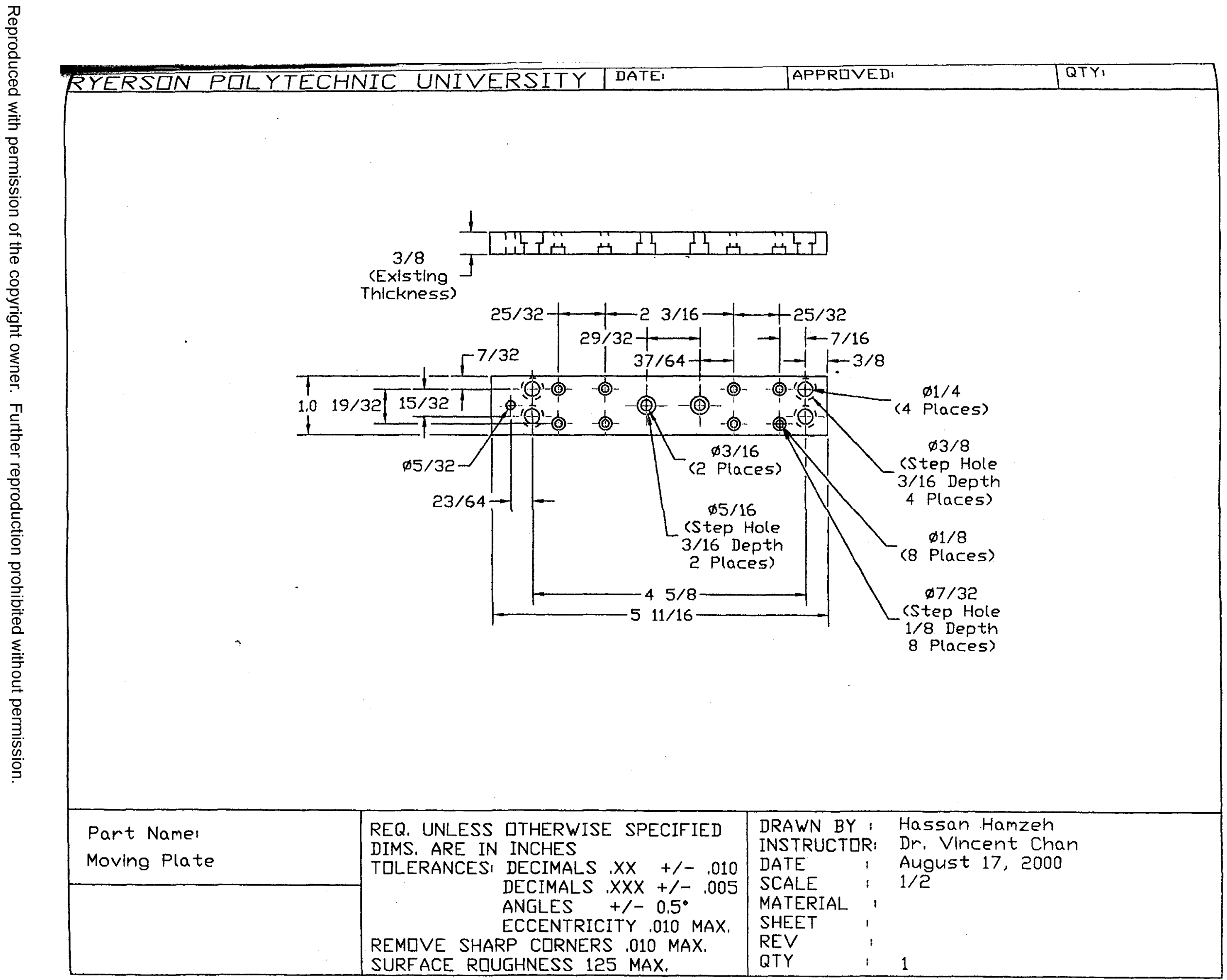




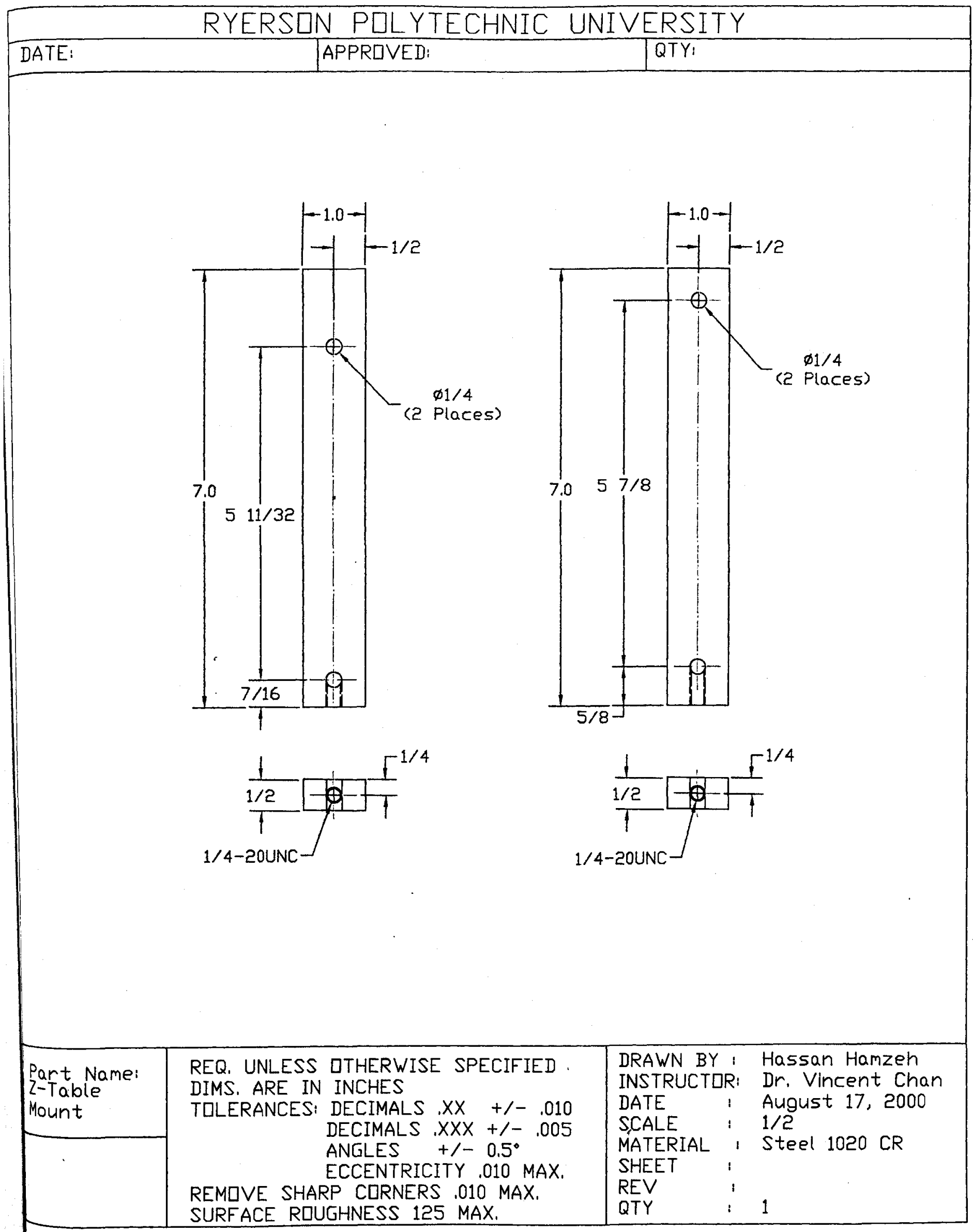




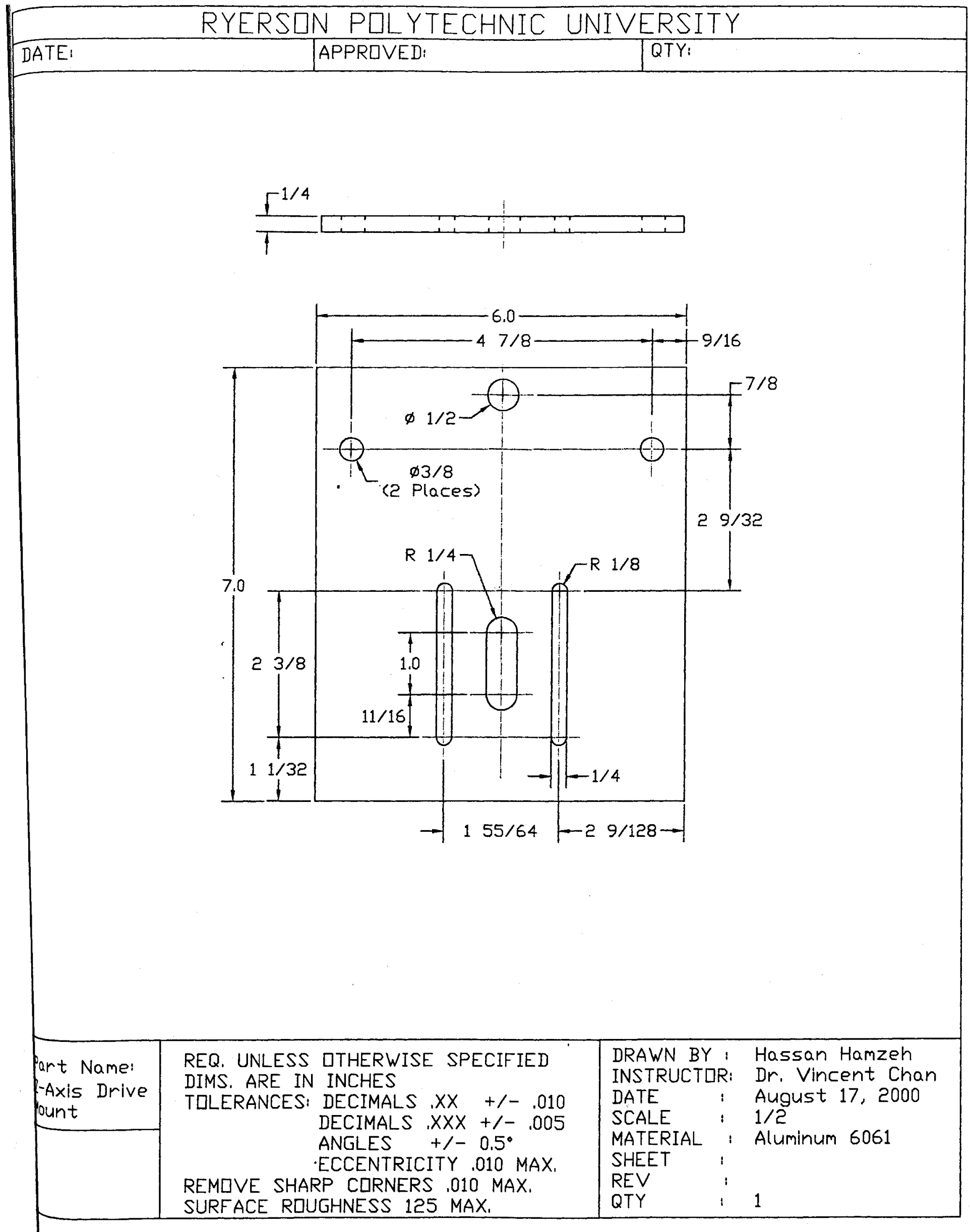




\section{APPENDIX 2}



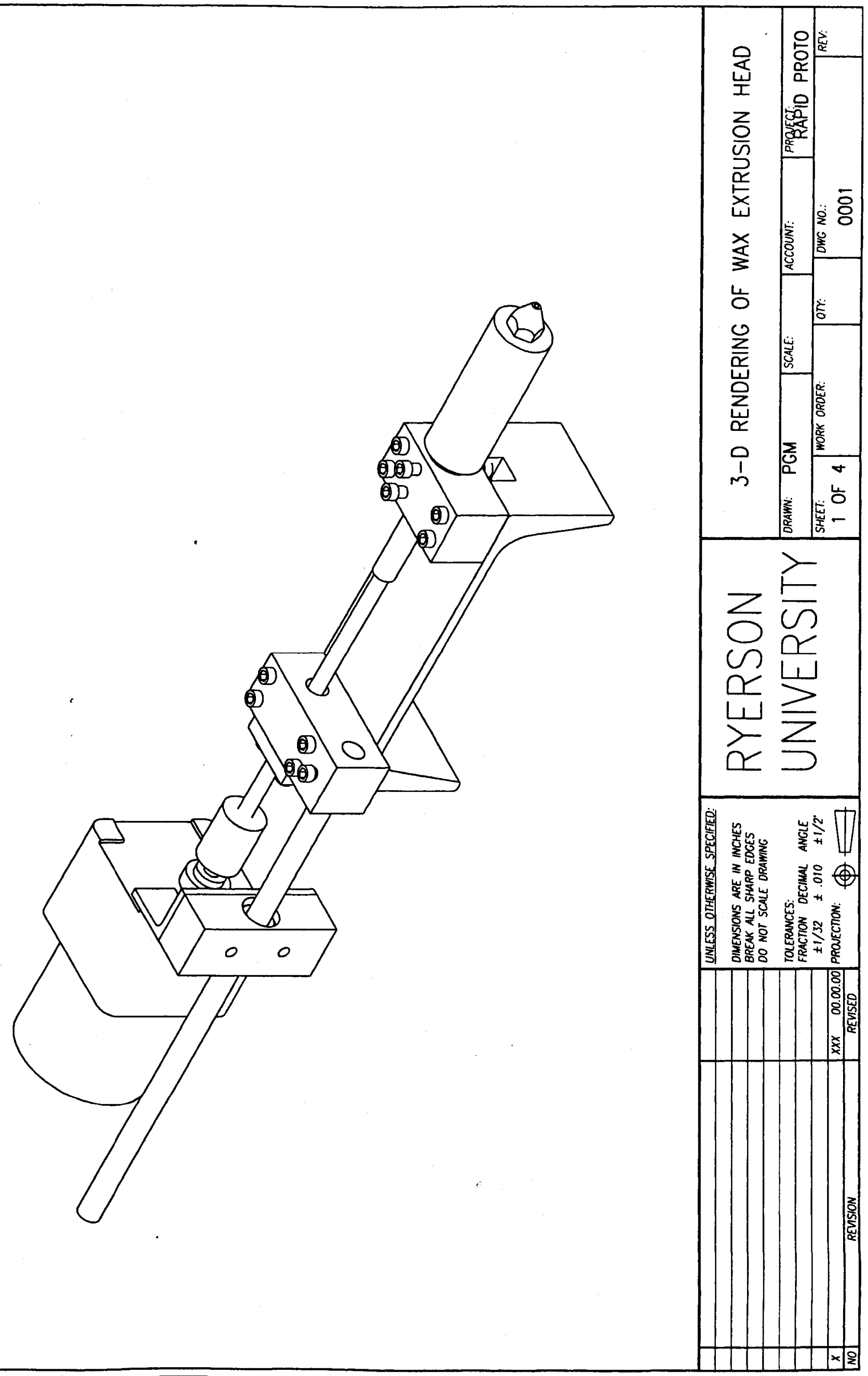


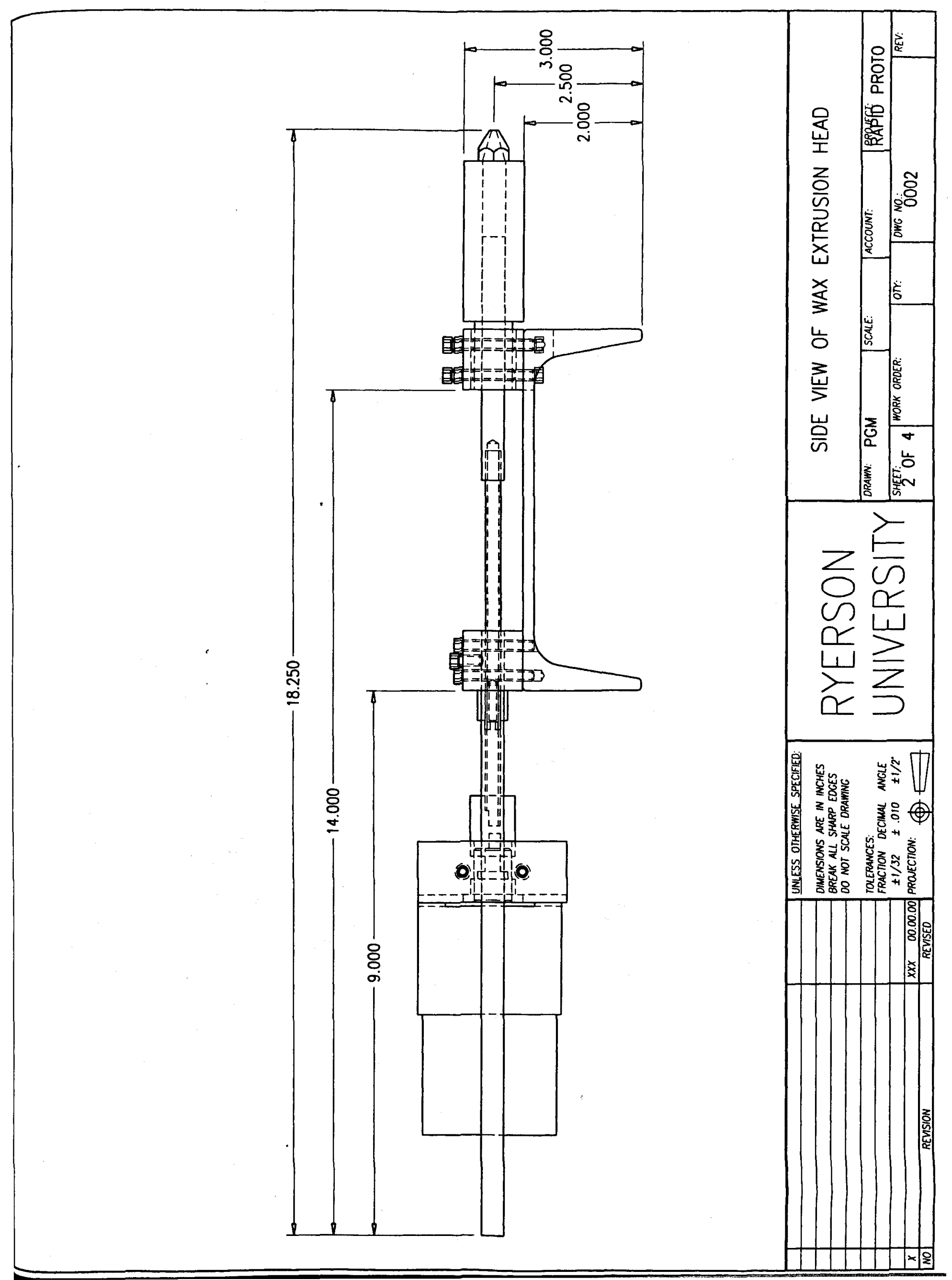

Reproduced with permission of the copyright owner. Further reproduction prohibited without permission. 

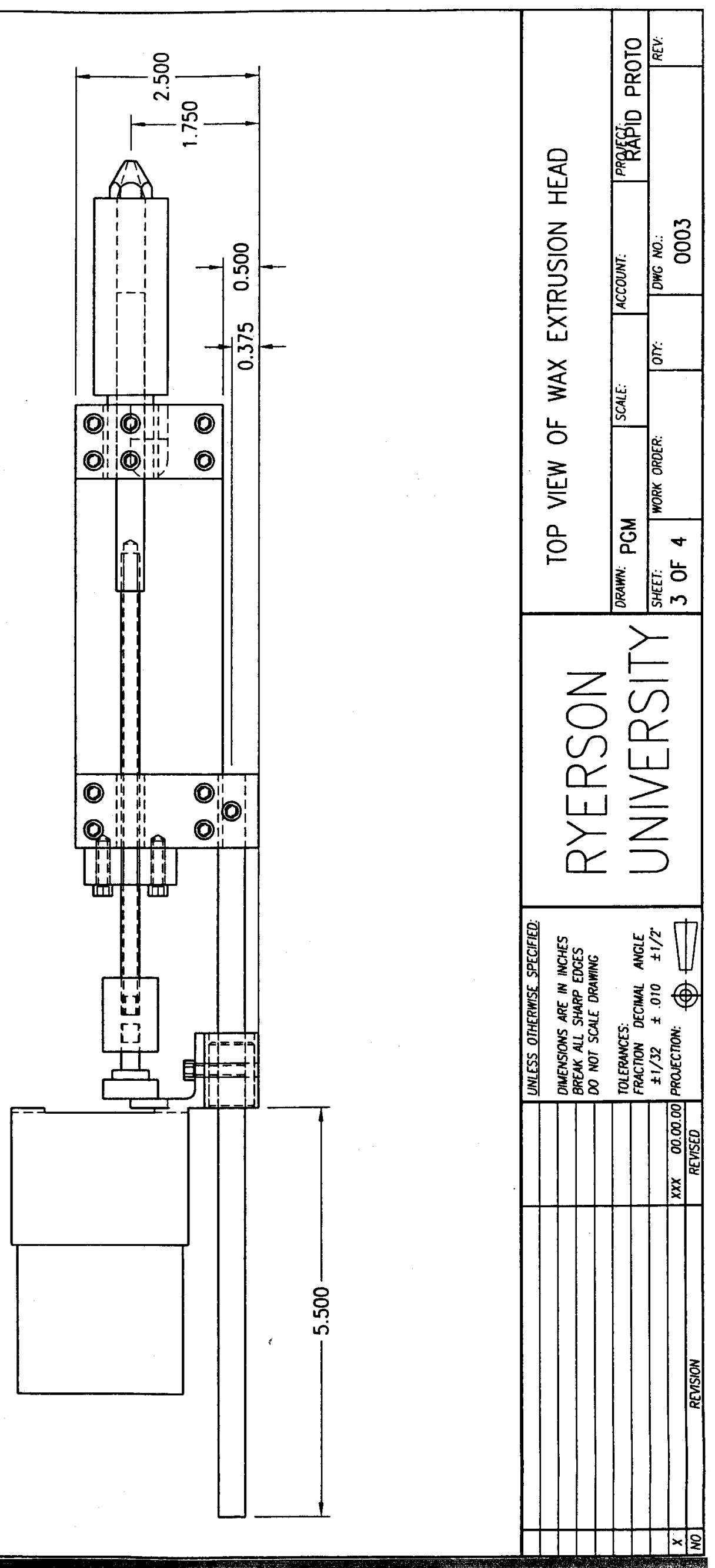


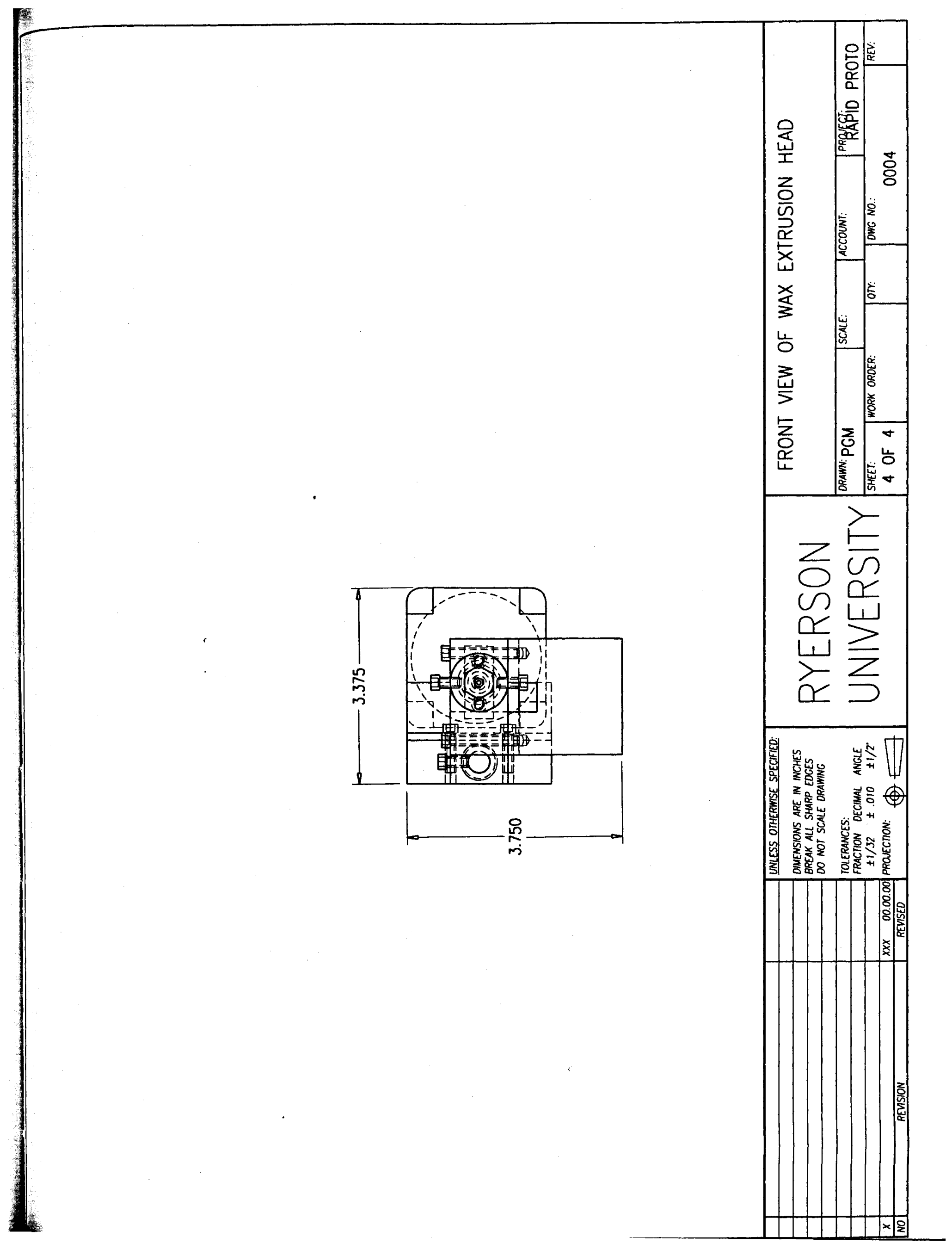

Reproduced with permission of the copyright owner. Further reproduction prohibited without permission. 


\section{APPENDIX 3}

Reproduced with permission of the copyright owner. Further reproduction prohibited without permission. 
' Program For Generating The G-Codes

' For a Cube or A Rectangular Prism

' Program \#0001

' Written By: Parham Ghasemi

Option Explicit

Private Sub cmdexit_Click()

End

End Sub

Private Sub cmdgcode_Click()

Dim xvalue As Currency

Dim xvalue2 As Currency

Dim xvalue3 As Currency

Dim moveup As Currency

Dim yvalue As Currency

Dim yvalue1 As Currency

Dim yvalue2 As Currency

Dim zvalue As Currency

Dim feedrate As Currency

Dim displacement As Currency

Dim layer As Variant

xvalue $=$ txtwidth. Text

xvalue2 $=($ xvalue -0$)$

yvalue $=$ txtlength.Text

yvalue 1 = txtlength.Text

zvalue $=$ txtheight.Text

feedrate $=$ txtfeedrate. Text

displacement $=$ txtdroplet. Text

layer $=$ txtdroplet.Text 
If xvalue $<4$ Then 'the working table is 4 inches by 4 inches

If yvalue $<4$ Then

txtdisplay.SelText = "G00 Z" \& layer $/ 2$ \& vbNewLine

Do Until layer / $2>$ zvalue 'Do until the given height is reached

txtdisplay.SelText = "M08" \& vbNewLine

txtdisplay.SelText = "G04 X30" \& vbNewLine ' Dwell for 3 seconds to allow the pump to start

Do Until yvalue <= yvalue2 'do until the given length is reached

' The following 4 commands generate one cycle of a layer

txtdisplay.SelText = "G01 X" \& (xvalue2 + 0.015) \& " Y" \& yvalue2 \& " Z" \& layer $/ 2$ \& " F" \& feedrate \& vbNewLine

yvalue 2 = yvalue $2+$ displacement

txtdisplay.SelText = "G01 X" \& (xvalue2 + 0.015) \& " Y" \& yvalue2 \& " Z" \& layer / 2 \& " F" \& feedrate \& vbNewLine

xvalue $3=($ xvalue -0$)+(0-$ xvalue $)$

txtdisplay.SelText = "G01 X" \& (xvalue3 - 0.015) \& " Y" \& yvalue2 \& " Z" \& layer / 2 \& " F" \& feedrate \& vbNewLine

yvalue $2=$ yvalue $2+$ displacement

txtdisplay.SelText = "G01 X" \& (xvalue3 - 0.015) \& " Y" \& yvalue2 \& " Z" \& layer $/ 2$ \& " F" \& feedrate \& vbNewLine

Loop The cycle is repeated until one layer is completed

' Linearly moves the wax head to the final point

txtdisplay.SelText = "G01 X" \& (xvalue2 + 0.015) \& " Y" \& yvalue2 \& " Z" \& layer $/ 2$ \& " F" \& feedrate \& vbNewLine

The Table is lowered 
txtdisplay.SelText = "M09" \& vbNewLine

moveup $=(1.8 *$ layer $/ 2)$

' The Wax head is rapidly transveresed back to the Home position

txtdisplay.SelText = "G00 Z" \& moveup \& " F" \& feedrate \& vbNewLine

txtdisplay.SelText = "G04 X300" \& vbNewLine ' Dwell for 30 seconds to allow the part to solidify

' G-Code for machining the perimeter of each layer deposited

txtdisplay.SelText $=$ vbNewLine

txtdisplay.SelText $=$ "G00 X" \& $-3.175 \&$ " Y" \& $-0.12 \&$ " F" \& feedrate \& vbNewLine

txtdisplay.SelText $=$ "G00 Z" \& $0.01 \&$ vbNewLine

txtdisplay.SelText = "G01 X" \& ((xvalue2 - 3.05) + 0.12) \& " Y" \& $(0-0.12) \&$

" Z" \& 0.01 \& " F" \& feedrate \& vbNewLine

txtdisplay.SelText $=$ "G01 X" \& ((xvalue2 - 3.05) + 0.12) \& " Y" \& (yvalue + 0.12) \& " Z" \& $0.01 \&$ " F" \& feedrate \& vbNewLine

txtdisplay.SelText = "G01 X" \& (-3.05 - 0.12) \& " Y" \& (yvalue + 0.12) \& " Z"

\& $0.01 \&$ " F" \& feedrate \& vbNewLine

txtdisplay.SelText $=$ "G01 X" \& (-3.05 - 0.12) \& " Y" \& $(0-0.12) \& " \mathrm{Z} " \&$

$0.01 \&$ " F" \& feedrate \& vbNewLine

txtdisplay.SelText = "G00 Z" \& moveup \& " F" \& feedrate \& vbNewLine

yvalue $2=0$

txtdisplay.SelText $=$ vbNewLine

' G-Code for machining the surface of each layer

txtdisplay.SelText = "G00 X" \& (xvalue3 - 3.05) \& " Y" \& (yvalue2) \& " Z" \& moveup \& " F" \& feedrate \& vbNewLine

txtdisplay.SelText = "G00 Z" \& Format\$(layer / 2.1, "\#,\#0.000") \& vbNewLine Do Until yvalue1 <= yvalue 2 'do until the given length is reached 
' The following 4 commands generate one cycle for the machining of a layer

txtdisplay.SelText = "G01 X" \& (xvalue2 - 3.05) \& " Y" \& (yvalue2) \& " Z" \&

Format\$(layer / 2.1, "\#,\#\#0.000") \& " F" \& feedrate \& vbNewLine

yvalue 2 = yvalue $2+$ displacement

txtdisplay.SelText = "G01 X" \& (xvalue2 - 3.05) \& " Y" \& (yvalue2) \& " Z" \&

Format\$(layer / 2.1, "\#,\#\#0.000") \& " F" \& feedrate \& vbNewLine

xvalue $3=($ xvalue -0$)+(0-$ xvalue $)$

txtdisplay.SelText = "G01 X" \& (xvalue3 - 3.05) \& " Y" \& (yvalue2) \& " Z" \&

Format\$(layer / 2.1, "\#,\#\#0.000") \& " F" \& feedrate \& vbNewLine

yvalue $2=$ yvalue $2+$ displacement

txtdisplay.SelText = "G01 X" \& (xvalue3 - 3.05) \& " Y" \& (yvalue2) \& " Z" \&

Format\$(layer / 2.1, "\#,\#\#0.000") \& " F" \& feedrate \& vbNewLine

Loop 'The cycle is repeated until one layer is completed

' Linearly moves the wax head to the final point

txtdisplay.SelText = "G01 X" \& (xvalue2 - 3.05) \& " Y" \& (yvalue2) \& " Z" \&

Format\$(layer / 2.1, "\#,\#\#0.000") \& " F" \& feedrate \& vbNewLine

The Table is lowered

txtdisplay.SelText = "M09" \& vbNewLine

' The Wax head is rapidly transveresed back to the Home $\{(x, y)=(0,0)\}$ position layer $=$ layer + displacement ' Increment for the next layer txtdisplay.SelText = "G00 Z" \& Format\$(layer / 2.1, "\#,\#\#0.000") \& " F" \&

feedrate \& vbNewLine

txtdisplay.SelText = "G00 X0 Y0" \& " F" \& feedrate \& vbNewLine

yvalue $2=0$ ' Initialize the $y$-value back to zero

Loop

Else 'Beep if length is larger than 4 inches

txtdisplay.SelText $=$ "The length has to be smaller than 4 inches!"

Beep

End If

Else 'Beep if length is width is larger than 4 inches 
txtdisplay.SelText = "The width has to be smaller than 4 inches!"

Beep

End If

End Sub

'Sub command for saving the data as a text file

'in order to transfer it to the DeskNC DOS program

Private Sub cmdsave_Click()

Dim strfilename As String

Dim strtext As String

Dim strfilter As String

Dim strbuffer As String

Dim filehandle\%

strfilter $="$ Text $\left.\left({ }^{*} .{ }^{*}\right)\right|^{*} . \operatorname{txt} \mid$ All files $\left.\left({ }^{*} .^{*}\right)\right|^{*} .{ }^{* \prime \prime}$

cdmain.Filter $=$ strfilter

cdmain.ShowSave

If cdmain.FileName <> " Then

strfilename $=$ cdmain.FileName

strtext $=$ txtdisplay. Text

filehandle $\%=$ FreeFile

Open strfilename For Output As \#filehandle\%

MousePointer $=$ vbHourglass

Print \#filehandle\%, strtext

MousePointer $=$ vbDefault

Close \#filehandle\%

End If

End Sub 
' Program For Generating The G-Codes

' For a Solid Cylindrical Shape

' Program \#0002

' Written By: Parham Ghasemi

Option Explicit

Private Sub cmdexit_Click()

End

End Sub

Private Sub cmdgcode_Click()

Dim layer As Variant

Dim radius As Currency

Dim radius2 As Currency

Dim displacement As Currency

Dim diameter As Currency

Dim feedrate As Currency

Dim moveup As Currency

Dim zvalue As Currency

Dim constant As Currency

radius $=$ txtradius.. Text

displacement $=$ txtdroplet.Text

layer $=$ txtdroplet. Text

feedrate $=$ txtfeedrate. Text

zvalue $=$ txtheight.. ext

If radius $<4$ Then

If radius $>0$ Then 
Do Until layer $/ 2>$ zvalue

txtdisplay.SelText = "G00 Z" \& layer $/ 2$ \& vbNewLine

' Drops first layer of wax

Do Until radius < displacement

txtdisplay.SelText = "G00 X" \& radius \& " Z" \& layer / 2 \& " F" \& feedrate \& vbNewLine

txtdisplay.SelText = "G03 I" \& -radius \& " Z" \& layer / 2 \& " F" \& feedrate \& vbNewLine

radius $=$ radius - displacement

Loop

txtdisplay.SelText $=\mathrm{vbNewLine}$

' Transverse milling head into position

moveup $=(2 *($ layer $/ 2))$

txtdisplay.SelText = "G00 Z" \& moveup \& " F" \& feedrate \& vbNewLine

txtdisplay.SelText $=$ "G04 X300" \& vbNewLine ' Dwell for 30 seconds

'Travel to milling head point

txtdisplay.SelText = "G00 X" \& -3.05 \& " Y" \& 0 \& " F" \& feedrate \& vbNewLine txtdisplay.SelText = "G00 Z" \& Format\$(layer / 1.5, "\#,\#\#0.000") \& vbNewLine

' Machine the surface of the cylinder block

radius $=$ txtradius . Text

Do Until radius < (diameter - displacement)

diameter $=0.25^{\prime} 0.25^{\prime \prime}$ end mill

txtdisplay.SelText = "G00 X" \& (-3.05 + radius) \& " Z" \& Format\$(layer / 1.5, "\#,\#\#0.000") \& " F" \& feedrate \& vbNewLine

txtdisplay.SelText = "G03 I" \& (-radius) \& " Z" \& Format\$(layer / 1.5, "\#,\#\#0.000") \& " F" \& feedrate \& vbNewLine

radius $=$ radius - displacement

Loop 

' Machining the Perimeter of the Cylinder Block
txtdisplay.SelText $=$ vbNewLine
radius $=$ txtradius. Text

txtdisplay.SelText = "G00 Z" \& moveup \& vbNewLine

txtdisplay.SelText $=$ "G00 X" \& $(-3.05+$ (radius + 0.12)) \& "F" \& feedrate \& vbNewLine

txtdisplay.SelText = "G00 Z" \& 0.01 \& vbNewLine

txtdisplay.SelText = "G03 I" \& (-radius - 0.12) \& " Z" \& layer / 2 \& " F" \& feedrate \& vbNewLine

txtdisplay.SelText $=$ vbNewLine

layer $=$ layer + displacement

radius $=$ txtradius. Text

Loop

txtdisplay.SelText = "G00 X0 Y0" \& " F" \& feedrate \& vbNewLine

Else

txtdisplay.SelText = " The radius has to be larger than 0!"

Beep

End If

Else

txtdisplay.SelText = " The radius has to be smaller than 4!"

Beep

End If

End Sub

' Sub program for saving the data as

' a text file

Private Sub cmdsave_Click()

Dim strfilename As String

Dim strtext As String 


\author{
Dim strfilter As String \\ Dim strbuffer As String \\ Dim filehandle\% \\ strfilter $="$ Text $\left.\left({ }^{*} .{ }^{*}\right)\right|^{*} . \operatorname{txt} \mid$ All files $\left.\left({ }^{*} .{ }^{*}\right)\right|^{*} . * "$ \\ cdmain.Filter $=$ strfilter \\ cdmain.ShowSave \\ If cdmain.FileName <> "" Then \\ strfilename $=$ cdmain . FileName \\ strtext $=$ txtdisplay.. Text \\ filehandle $\%=$ FreeFile \\ Open strfilename For Output As \#filehandle\% \\ MousePointer $=$ vbHourglass \\ Print \#filehandle\%, strtext \\ MousePointer $=$ vbDefault \\ Close \#filehandle\% \\ End If \\ End Sub
}


' Program For Generating The G-Codes

' For a hollow Cube

' "A cube Within A cube"

' Program \#0003

' Written By: Parham Ghasemi

Option Explicit

Private Sub cmdexit_Click()

End

End Sub

Private Sub cmdgcode_Click0

Dim xvalue As Currency

Dim xvalue2 As Currency

Dim xvalue3 As Currency

Dim xvalue4 As Currency

Dim innerxvalue As Currency

Dim yvalue As Currency

Dim yvalue2 As Currency

Dim yvalue3 As Currency

Dim yvalue4 As Currency

Dim inneryvalue As Currency

Dim zvalue As Currency

Dim feedrate As Currency

Dim displacement As Currency

Dim layer As Variant

Dim moveup As Currency

Dim jumper As Currency

Dim jump As Currency 
xvalue $=$ txtwidth. Text

innerxvalue $=$ txtinnerwidth. Text

yvalue $=$ txtlength. Text

inneryvalue $=$ txtinnerlength. Text

zvalue $=$ txtheight.Text

feedrate $=$ txtfeedrate. Text

displacement $=$ txtdroplet. Text

layer $=$ txtdroplet.Text

If xvalue $<4$ Then ' the working table is 4 inches by 4 inches

If yvalue $<4$ Then

Do Until (layer / 2) >= zvalue ' Do until the given height is reached

txtdisplay.SelText = "G00 Z" \& layer / 2 \& vbNewLine

txtdisplay.SelText = "G04 X30" \& vbNewLine

Do While jumper $<=(($ yvalue - inneryvalue $) / 2)$ ' do until the given length is reached

' The following 4 commands generate one cycle of a layer

txtdisplay.SelText = "G00 X" \& xvalue4 \& " Y" \& yvalue4 \& " Z" \& layer / 2 \& " F" \& feedrate \& vbNewLine

xvalue 2 = xvalue - jumper

txtdisplay.SelText = "G01 X" \& xvalue2 \& " Y" \& yvalue4 \& " Z" \& layer / 2 \& " F" \& feedrate \& vbNewLine

$$
\text { yvalue } 2 \text { = yvalue }- \text { jumper }
$$

txtdisplay.SelText = "G01 X" \& xvalue2 \& " Y" \& yvalue2 \& " Z" \& layer / 2 \& " F" \& feedrate \& vbNewLine

$$
\text { xvalue } 3=\text { xvalue } 3+\text { jumper }
$$

txtdisplay.SelText = "G01 X" \& xvalue3 \& " Y" \& yvalue2 \& " Z" \& layer / 2 \& " F" \& feedrate \& vbNewLine

$$
\text { yvalue } 3=\text { yvalue } 3+\text { jumper }
$$

txtdisplay.SelText = "G01 X" \& xvalue3 \& " Y" \& yvalue3 \& " Z" \& layer / 2 \& " F" \& feedrate $\&$ vbNewLine 
xvalue 4 = xvalue 4 + displacement

yvalue 4 = yvalue $4+$ displacement

jumper $=$ jumper + displacement

xvalue $3=0$

yvalue $3=0$

Loop The cycle is repeated until one layer is completed

txtdisplay.SelText $=$ vbNewLine

The Table is lowered

moveup $=(2 *($ layer $/ 2))$

txtdisplay.SelText = "G00 Z" \& moveup \& vbNewLine

' The Wax head is rapidly transveresed back to the Home position

txtdisplay.SelText = "G04 X300" \& vbNewLine

txtdisplay.SelText $=$ "G00 X" \& $-3.05 \&$ \& $Y " \& 0$ \& " F" \& feedrate \& vbNewLine

txtdisplay.SelText = "G00 Z" \& Format\$(layer / 2.1, "\#,\#\#0.000")

txtdisplay.SelText $=$ vbNewLine

'Machining The Surface of the Square

xvalue $2=0$

xvalue $4=0$

yvalue $2=0$

yvalue $4=0$

Do While jump $<=(($ yvalue - inneryvalue $) / 2)$

txtdisplay.SelText $=$ "G00 X" \& (xvalue4 - 3.05) \& " Y" \& yvalue4 \& " Z" \& Format\$(layer / 2.1, "\#,\#\#0.000") \& " F" \& feedrate \& vbNewLine

xvalue 2 = xvalue - jump 
txtdisplay.SelText = "G01 X" \& (xvalue2 - 3.05) \& " Y" \& yvalue4 \& " Z" \& Format $\$($ layer / 2.1, "\#,\#\#0.000") \& " F" \& feedrate \& vbNewLine

yvalue 2 = yvalue - jump

txtdisplay.SelText = "G01 X" \& (xvalue2 - 3.05) \& " Y" \& yvalue2 \& " Z" \& Format $\$$ (layer / 2.1, "\#,\#\#0.000") \& " F" \& feedrate \& vbNewLine

xvalue $3=$ xvalue $3+$ jump

txtdisplay.SelText = "G01 X" \& (xvalue3 - 3.05) \& " Y" \& yvalue2 \& " Z" \& Format $\$($ layer / 2.1, "\#,\#\#0.000") \& " F" \& feedrate \& vbNewLine

yvalue $3=$ yvalue $3+$ jump

txtdisplay.SelText = "G01 X" \& (xvalue3 - 3.05) \& " Y" \& yvalue3 \& " Z" \& Format $\$($ layer / 2.1, "\#,\#\#0.000") \& " F" \& feedrate \& vbNewLine

jump $=$ jump + displacement

xvalue $4=$ xvalue $4+$ displacement

yvalue $4=$ yvalue $4+$ displacement

xvalue $3=0$

yvalue $3=0$

Loop

txtdisplay.SelText $=$ vbNewLine

'Machining The Perimeter of the Outside Square

txtdisplay.SelText $=$ "G00 X" \& $-3.17 \&$ " Y" \& $-0.12 \&$ " F" \& feedrate \& vbNewLine

txtdisplay.SelText = "G00 Z" \& $0.01 \&$ " F" \& feedrate \& vbNewLine

txtdisplay.SelText $=$ "G01 X" \& (xvalue - 3.05 + 0.12) \& " Y" \& $-0.12 \&$ " F" \&

feedrate \& vbNewLine

txtdisplay.SelText = "G01 X" \& (xvalue - 3.05 + 0.12) \& " Y" \& (yvalue + 0.12)

\& " F" \& feedrate \& vbNewLine 
txtdisplay.SelText $=$ "G01 X" \& $-3.17 \&$ " Y" \& (yvalue + 0.12) \& " F" \& feedrate $\&$ vbNewLine txtdisplay.SelText = "G01 X" \& $-3.17 \&$ " Y" \& $-0.12 \&$ " F" \& feedrate \& vbNewLine

txtdisplay.SelText = "G00 Z" \& moveup \& vbNewLine

txtdisplay.SelText $=$ vbNewLine

' Machining The Perimeter of the Inside Square

txtdisplay.SelText = "G00 X" \& (xvalue4 - 3.05) \& " Y" \& (yvalue4) \& " F" \& feedrate \& vbNewLine

txtdisplay.SelText $=$ "G00 Z" \& $0.01 \&$ vbNewLine

txtdisplay.SelText = "G01 X" \& (xvalue2 - 3.05 - 0.12) \& " Y" \& (yvalue4) \& "

F" \& feedrate \& vbNewLine

txtdisplay.SelText = "G01 X" \& (xvalue2 - 3.05 - 0.12) \& " Y" \& (yvalue2 0.12) \& " F" \& feedrate \& vbNewLine

txtdisplay.SelText = "G01 X" \& (xvalue4 - 3.05) \& " Y" \& (yvalue2 - 0.12) \& "

$F^{\prime \prime} \&$ feedrate \& vbNewLine

txtdisplay.SelText = "G01 X" \& (xvalue4 - 3.05) \& " Y" \& (yvalue4) \& " F" \& feedrate \& vbNewLine

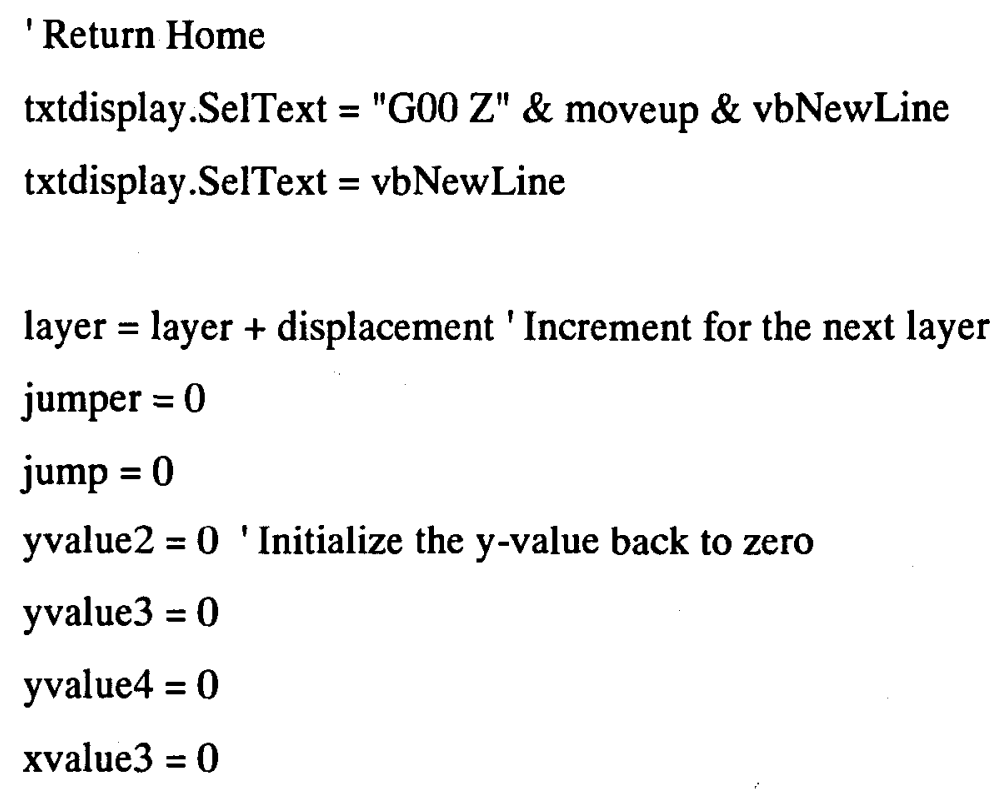




$$
\text { xvalue } 4=0
$$

Loop

Else

txtdisplay.SelText = "The length has to be smaller than 4 inches!"

Beep

End If

Else

txtdisplay.SelText = "The width has to be smaller than 4 inches!"

Beep

End If

End Sub

Private Sub cmdsave_Click 0

Dim strfilename As String

Dim strtext As String

Dim strfilter As String

Dim strbuffer As String

Dim filehandle\%

strfilter $="$ Text $\left.\left({ }^{*} \cdot{ }^{*}\right)\right|^{*} . \operatorname{txt} \mid$ All files $\left.\left({ }^{*} \cdot{ }^{*}\right)\right|^{*} .^{* \prime}$

cdmain.Filter $=$ strfilter

cdmain.ShowSave

If cdmain.FileName <> "" Then

strfilename $=$ cdmain.FileName

strtext $=$ txtdisplay. Text

filehandle $\%=$ FreeFile

Open strfilename For Output As \#filehandle\%

MousePointer $=$ vbHourglass

Print \#filehandle\%, strtext 
MousePointer $=$ vbDefault

Close \#filehandle\%

End If

End Sub 
' Program For Generating The G-Codes

' For a Pyramidal Shape

' Program \#0004

' Written By: Parham Ghasemi

Option Explicit

Private Sub cmdexit_Click()

End

End Sub

Private Sub cmdgcode_Click $(0$

Dim xvalue As Currency

Dim xvalue2 As Currency

Dim xvalue3 As Currency

Dim moveup As Currency

Dim yvalue As Currency

Dim yvalue1 As Currency

Dim yvalue2 As Currency

Dim zvalue As Currency

Dim feedrate As Currency

Dim displacement As Currency

Dim layer As Variant

Dim steps As Currency

xvalue $=$ txtwidth.Text

xvalue $2=($ xvalue -0$)$

yvalue $=$ txtlength.Text

yvalue $1=$ txtlength. Text

zvalue $=$ txtheight. Text

feedrate $=$ txtfeedrate. Text

displacement $=$ txtdroplet. Text

layer $=$ txtdroplet.Text 
steps $=$ zvalue $/ 3$

If xvalue $<4$ Then ' the working table is 4 inches by 4 inches

If yvalue $<4$ Then

Do Until layer / $2>$ zvalue ' Do until the given height is reached txtdisplay.SelText = "G00 Z" \& layer $/ 2$ \& vbNewLine txtdisplay.SelText $=$ "G04 X30" \& vbNewLine

Do Until yvalue $<=$ yvalue2 'do until the given length is reached

' The following 4 commands generate one cycle of a layer

txtdisplay.SelText = "G01 X" \& xvalue2 \& " Y" \& yvalue2 \& " Z" \& layer $/ 2$ \&

" F" \& feedrate \& vbNewLine

yvalue 2 = yvalue $2+$ displacement

txtdisplay.SelText = "G01 X" \& xvalue2 \& " Y" \& yvalue2 \& " Z" \& layer / 2 \&

" F" \& feedrate \& vbNewLine

xvalue $3=($ xvalue -0$)+(0-$ xvalue $)$

txtdisplay.SelText = "G01 X" \& xvalue3 \& " Y" \& yvalue2 \& " Z" \& layer / 2 \&

" F" \& feedrate \& vbNewLine

yvalue 2 = yvalue $2+$ displacement

txtdisplay.SelText = "G01 X" \& xvalue3 \& " Y" \& yvalue2 \& " Z" \& layer $/ 2$ \&

" F" \& feedrate \& vbNewLine

Loop The cycle is repeated until one layer is completed

'Linearly moves the wax head to the final point

txtdisplay.SelText = "G01 X" \& xvalue2 \& " Y" \& yvalue2 \& " Z" \& layer / 2 \&

" F" \& feedrate \& vbNewLine

The Table is lowered

txtdisplay.SelText $=$ vbNewLine

moveup $=1.8 *($ layer $/ 2)$

' The Wax head is rapidly transveresed back to the Home position 
txtdisplay.SelText = "G00 Z" \& moveup \& " F" \& feedrate \& vbNewLine txtdisplay.SelText = "G04 X300" \& vbNewLine

' G-Code for machining the perimeter of each layer deposited

txtdisplay.SelText = "G00 X" \& $-3.17 \&$ " Y" \& $-0.12 \&$ " F" \& feedrate \& vbNewLine

txtdisplay.SelText = "G00 Z" \& $0.01 \&$ vbNewLine

txtdisplay.SelText = "G01 X" \& (xvalue2 - 3.05 + 0.12) \& " Y" \& $(0$ - 0.12) \& " $F^{\prime \prime} \&$ feedrate \& vbNewLine

txtdisplay.SelText = "G01 X" \& (xvalue2 - $3.05+0.12) \&$ " Y" \& (yvalue + 0.12) \& " F" \& feedrate \& vbNewLine

txtdisplay.SelText $=$ "G01 X" \& (-3.05 - 0.12) \& " Y" \& (yvalue + 0.12) \& " F" $\&$ feedrate \& vbNewLine

txtdisplay.SelText $=$ "G01 X" \& (-3.05 - 0.12) \& " Y" \& $(0-0.12) \&$ " F" \& feedrate $\&$ vbNewLine

txtdisplay.SelText = "G00 Z" \& moveup \& " F" \& feedrate \& vbNewLine txtdisplay.SelText $=$ vbNewLine

yvalue $2=0$

' G-Code for machining the surface of each layer

txtdisplay.SelText $=$ "G00 X" \& (xvalue3 - 3.05) \& " Y" \& yvalue2 \& " F" \& feedrate $\&$ vbNewLine

txtdisplay.SelText = "G00 Z" \& Format\$(layer / 1.6, "\#,\#\#0.000") \& vbNewLine

Do Until yvalue1 <= yvalue2 'do until the given length is reached 
' The following 4 commands generate one cycle for the machining of a layer

txtdisplay.SelText = "G01 X" \& (xvalue2 - 3.05) \& " Y" \& yvalue2 \& " Z" \& Format $\$($ layer / 1.6, "\#,\#\#0.000") \& " F" \& feedrate \& vbNewLine

yvalue 2 = yvalue $2+$ displacement

txtdisplay.SelText = "G01 X" \& (xvalue2 - 3.05) \& " Y" \& yvalue2 \& " Z" \&

Format $\$($ layer / 1.6, "\#,\#\#0.000") \& " F" \& feedrate \& vbNewLine

xvalue $3=($ xvalue -0$)+(0-$ xvalue $)$

txtdisplay.SelText = "G01 X" \& (xvalue3 - 3.05) \& " Y" \& yvalue2 \& " Z" \& Format $\$($ layer / 1.6, "\#,\#\#0.000") \& " F" \& feedrate \& vbNewLine

yvalue $2=$ yvalue $2+$ displacement

txtdisplay.SelText = "G01 X" \& (xvalue3 - 3.05) \& " Y" \& yvalue2 \& " Z" \& Format $\$$ (layer / 1.6, "\#,\#\#0.000") \& " F" \& feedrate \& vbNewLine

Loop The cycle is repeated until one layer is completed

' Linearly moves the wax head to the final point

txtdisplay.SelText = "G01 X" \& (xvalue2 - 3.05) \& " Y" \& yvalue2 \& " Z" \& Format $\$$ (layer / 1.6, "\#,\#\#0.000") \& " F" \& feedrate \& vbNewLine

The Table is lowered

txtdisplay.SelText $=$ vbNewLine

moveup $=1.8 *($ layer / 2)

' The Wax head is rapidly transveresed back to the Home $\{(x, y)=(0,0)\}$ position txtdisplay.SelText = "G00 Z" \& moveup \& " F" \& feedrate \& vbNewLine txtdisplay.SelText = "G00 X0 Y0" \& vbNewLine

layer = layer + displacement ' Increment for the next layer

yvalue $2=0$ ' Initialize the $y$-value back to zero

Loop 
Else 'Beep if length is larger than 4 inches

txtdisplay.SelText = "The length has to be smaller than 4 inches!"

Beep

End If

Else 'Beep if length is width is larger than 4 inches

txtdisplay.SelText = "The width has to be smaller than 4 inches!"

Beep

End If

' Machine two steps to resemble a pyramid like shape

' The steps are pre-determined

txtdisplay.SelText = "G00 X" \& (-3.05 + 0.125) \& " Y" \& -0.12 \& " Z" \& steps \& " F" \& feedrate \& vbNewLine

txtdisplay.SelText = "G01 X" \& (-3.05 + xvalue2 - 0.12) \& " Y" \& $0.12 \&$ " Z" \& steps $\&$ " F" \& feedrate \& vbNewLine

txtdisplay.SelText = "G01 X" \& (-3.05 + xvalue2 - 0.12) \& " Y" \& (yvalue - 0.12) \& " Z" \& steps \& " F" \& feedrate \& vbNewLine

txtdisplay.SelText = "G01 X" \& (-3.05 + 0.12) \& " Y" \& (yvalue - 0.12) \& " Z" \& steps $\&$ " F" \& feedrate $\&$ vbNewLine

txtdisplay.SelText = "G01 X" \& (-3.05 + 0.12) \& " Y" \& (-0.12) \& " Z" \& steps \& " F" \& feedrate \& vbNewLine

txtdisplay.SelText = "G00 Z" \& (steps * 2) \& " F" \& feedrate \& vbNewLine

txtdisplay.SelText = "G00 X" \& (-3.05 + 0.375) \& " Y" \& $0.375 \&$ " Z" \& (steps * 2) \& " $F^{\prime \prime} \&$ feedrate \& vbNewLine

txtdisplay.SelText = "G01 X" \& (-3.05 + xvalue2 - 0.375) \& " Y" \& $0.375 \&$ " Z" \& (steps * 2) \& " F" \& feedrate \& vbNewLine

txtdisplay.SelText = "G01 X" \& (-3.05 + xvalue2 - 0.375) \& " Y" \& (yvalue - 0.375) \& " Z" \& (steps * 2) \& " F" \& feedrate \& vbNewLine 
txtdisplay.SelText = "G01 X" \& (-3.05 + 0.375) \& " Y" \& (yvalue - 0.375) \& " Z" \& (steps * 2) \& " F" \& feedrate \& vbNewLine

txtdisplay.SelText $=$ "G01 X" \& $(-3.05+0.375) \&$ " Y" \& $(0+0.375) \&$ " Z" \& (steps * 2) \& " F" \& feedrate \& vbNewLine

txtdisplay.SelText = "G00 Z" \& moveup \& feedrate \& vbNewLine

txtdisplay.SelText = "G00 X0 Y0" \& vbNewLine

End Sub

'Sub command for saving the data as a text file

'in order to transfer it to the DeskNC DOS program

Private Sub cmdsave_Click0

Dim strfilename As String

Dim strtext As String

Dim strfilter As String

Dim strbuffer As String

Dim filehandle\%

strfilter $="$ Text $\left.\left({ }^{*} . *\right)\right|^{*} . \operatorname{txt} \mid$ All files $\left.\left({ }^{*} .{ }^{*}\right)\right|^{*} .{ }^{*} "$

cdmain.Filter $=$ strfilter

cdmain.ShowSave

If cdmain.FileName <> "" Then

strfilename $=$ cdmain. FileName

strtext $=$ txtdisplay. Text

filehandle\% $=$ FreeFile

Open strfilename For Output As \#filehandle\%

MousePointer $=$ vbHourglass

Print \#filehandle\%, strtext

MousePointer $=$ vbDefault

Close \#filehandle\%

End If

End Sub 